Phase II SBIR Final Report:

\title{
Joining of Tungsten Armor Using Functional Gradients
}

DOE Grant No.: DE-FG02-96ER82257

Dates:

August 13, 1996 - June 27, 2002

\author{
Scott O’Dell \\ and
}

Timothy McKechnie

Plasma Processes, Inc.

4914 Moores Mill Road

Huntsville, Alabama 35811

(256) 851-7653 


\begin{abstract}
The joining of low thermal expansion armor materials such as tungsten to high thermal expansion heat sink materials has been a major problem in plasma facing component (PFC) development. Conventional planar bonding techniques have been unable to withstand the high thermal induced stresses resulting from fabrication and high heat flux testing. During this investigation, innovative functional gradient joints produced using vacuum plasma spray forming techniques have been developed for joining tungsten armor to copper alloy heat sinks. A model was developed to select the optimum gradient architecture. Based on the modeling effort, a $2 \mathrm{~mm}$ copper rich gradient was selected. Vacuum plasma pray parameters and procedures were then developed to produce the functional gradient joint. Using these techniques, dual cooling channel, medium scale mockups (32mm wide x 400mm length) were produced with vacuum plasma spray formed tungsten armor. The thickness of the tungsten armor was up to $5 \mathrm{~mm}$ thick. No evidence of debonding at the interface between the heat sink and the vacuum plasma sprayed material was observed.
\end{abstract}

\title{
SBIR DATA RIGHTS
}

These SBIR data are furnished with SBIR rights under Grant No. DE-FG02-96ER82257. For a period of 4 years after acceptance of all items to be delivered under this grant, the Government agrees to use these data for Government purposes only, and they shall not be disclosed outside the Government (including disclosure for procurement purposes) during such period without permission of the grantee, except that, subject to the foregoing use and disclosure prohibitions, such data may be disclosed for use by support contractors. After the aforesaid 4-year period, the Government has a royalty-free license to use, and to authorize others to use on it behalf, these data for Government purposes, but is relieved of all disclosure prohibitions and assumes no liability for unauthorized use of these data by third parties. This Notice shall be affixed to any reproductions of these data in whole or in part.

\section{TABLE OF CONTENTS}

$\begin{array}{lll}\text { Abstract } & \text { i }\end{array}$

SBIR Data Rights $\quad$ i

1.0 Identification and Significance of the Phase II Investigation 1

2.0 Modeling of Cu-W Functional Gradient Joint 4

3.0 Mechanical and Physical Properties of VPS Deposits 34

4.0 Medium Scale Mockup Development 53

$\begin{array}{lll}5.0 & \text { Summary } & 64\end{array}$

$\begin{array}{lll}6.0 & \text { References } & 65\end{array}$

$\begin{array}{ll}\text { Appendix A } & 67\end{array}$

$\begin{array}{ll}\text { Appendix B } & 92\end{array}$ 


\subsection{IDENTIFICATION AND SIGNIFICANCE OF THE PHASE II INVESTIGATION}

\subsection{Identification of the Need for Joining Tungsten Armor to Copper}

The Department of Energy and the International Thermonuclear Experimental Reactor (ITER) program are searching for techniques to join and to repair plasma facing components (PFCs) in magnetic fusion energy devices. A PFC is comprised of an armor material, which is in contact with the high energy plasma, and a heat sink, which is actively cooled by high velocity fluids passing through internal cooling channels. Figure 1 is a schematic representation of a PFC. The armor material must have a significant melting temperature so as to withstand the high plasma temperature encountered.

The materials presently being considered for PFC armor are beryllium, carbon-carbon composites and tungsten. Due to its high melting temperature, good conductivity, disruption resistance, melt layer stability and low sputtering rate, tungsten is considered an excellent candidate for the armor material. Presently, DOE is considering a copper alloy as the heat sink because of its high thermal conductivity. The combination of copper alloys and tungsten alloys is ideal for high heat flux applications such as ITER.

Tungsten, positioned on the hot side, has the capability to withstand extremely high temperatures while maintaining its mechanical properties. $\mathrm{Cu}$, positioned on the cool side, has the ability to conduct heat to the cooling channels at a higher heat transfer rate than do most other materials. Until recently the ability to metallurgically join these materials with low residual joint stresses has eluded would be users, due to the drastic difference in the coefficient of thermal expansions $(\mathrm{Cu}=16.7 \mu \mathrm{m} / \mathrm{m} \mathrm{K}$ and $\mathrm{W}=4.5 \mu \mathrm{m} / \mathrm{m} \mathrm{K})$. However, the ability to vacuum plasma spray form functionally gradient materials allows materials with extreme differences in thermal expansion to be successfully joined. During this investigation, the feasibility of utilizing vacuum plasma spray techniques to join tungsten armor to copper alloy heat sinks employing functional gradients has been demonstrated. This process has been used to fabricate small and medium scale divertor mockups for high heat flux testing at Sandia National Laboratories.

\section{Armor and Heat Sink Diagram:}

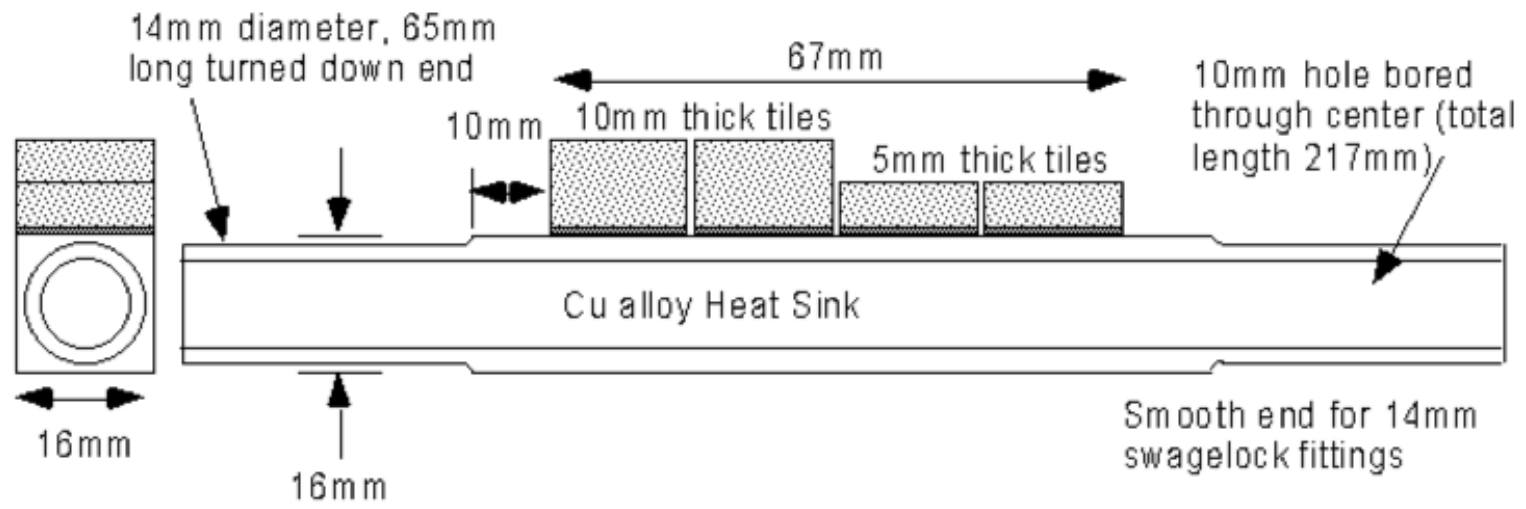

Figure 1 - Schematic representation of an EBTS small scale plasma facing component mockup for high heat flux testing at SNLs. 


\subsection{Background}

Tungsten is being baselined for critical PFC locations (i.e., curved gas box liner, divertor wing flat sides, divertor wing curved sides and baffle module). These locations are ideal for vacuum plasma sprayed tungsten because of the heat fluxes (1 to $3 \mathrm{MW} / \mathrm{m} 2)$ that will be encounter. An advantage of thermal spray technology such as vacuum plasma spray is the ability to fabricate components using high temperature materials (i.e., tungsten). Then by employing unique functional gradient techniques, vacuum plasma spraying can be utilized to join and to repair metals with dramatic differences in thermal expansion coefficients (i.e., copper, tungsten). The $\mathrm{Cu} / \mathrm{W}$ functional gradient maximizes integral bonding of the dissimilar metals while minimizing thermal stresses and strains that develop in conventionally bonded joints of materials with different thermal expansions. Also, the telerobotic control of the plasma spray process lends itself to essential repair and maintenance of radioactive fusion reactors. The high deposition rates ( $\mathrm{mm} /$ hour) of vacuum plasma spray is a major cost advantage over competing processes, such as CVD and PVD. VPS deposition on large curved surfaces has already been proven on large land based gas turbines by GE and Westinghouse, and on large rocket engines by NASA, Rocketdyne, and PPI. The plasma sprayed functional gradients can also be used for in-situ repair whereas a powder metallurgy/HIP gradient could not.

Vacuum plasma spraying involves depositing material onto a substrate to form a protective coating or a mandrel of the desired shape and subsequently removing the mandrel to form a nearnet-shape spray formed structure. A schematic representation of the plasma gun used during the VPS process is shown in Figure 2. Gases (usually Ar as the primary gas and $\mathrm{He}, \mathrm{H}_{2}$ or $\mathrm{N}_{2}$ as the secondary gas) are flowed between a tungsten cathode and a water-cooled tungsten lined, copper anode. An electric arc is initiated between the two electrodes and ionizes the gases creating a high pressure plasma. A measured quantity of powder feedstock is introduced into the gas stream either just outside the torch or in the diverging exit region of the nozzle (anode). Plasmafeedstock interactions lead to the formation of dense deposits. Advantages of the VPS process for producing tungsten armored copper alloy heat sinks are listed below:

- Processing of oxygen sensitive materials, i.e., copper and tungsten.

- Ability to use reactive gases

- High temperature plasma enables the processing of high melting temperature materials, i.e., W.

- $\mathrm{CNC}$ control enables contour following and uniform, repeatable deposition thicknesses.

- Rapid cooling rate and short time of flight in the plasma flame allows the retention of fine grained microstructures.

- Use of powder feedstock enables the fabrication of dispersion strengthened alloys.

- Ability to tailor the structure of the deposits by varying the spray parameters

o Functional gradient layers for joining materials with dissimilar CTEs

o Multi-layered structures

o Porous structures 


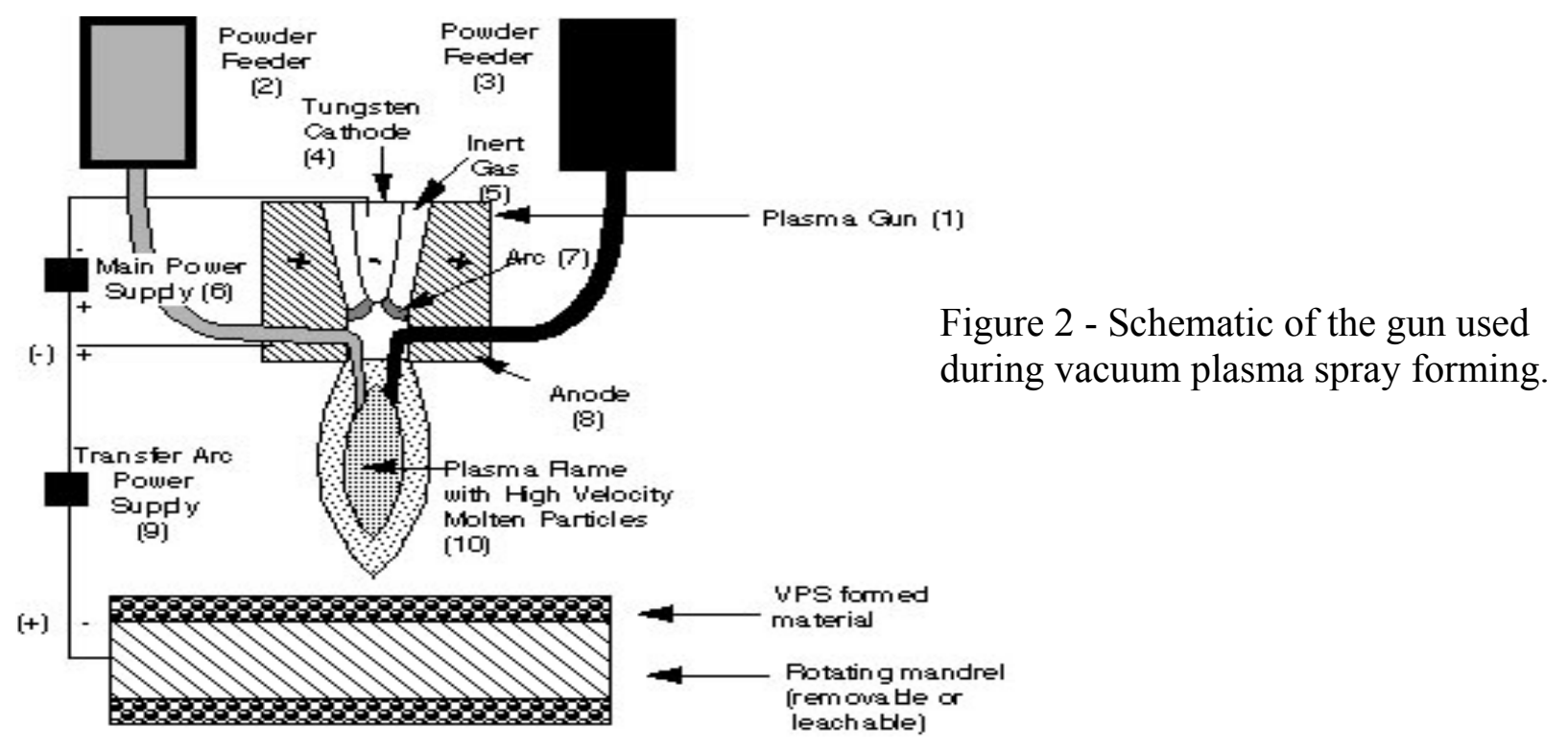

Figure 3 is photograph of one of three VPS systems used at PPI. Each system is equipped with a Sulzer/Metco/EPI $140 \mathrm{KW}$ Vacuum Plasma Spray gun modified with high efficiency nozzles. The following sections will detail the development of VPS tungsten armored copper alloy heat sinks. The initial step was to develop a finite element model to determine the optimum gradient architecture. In addition, processing parameters were developed to improve the properties of VPS tungsten and copper. Samples were produced for mechanical and physical properties testing of VPS formed tungsten and copper deposits. Based on the results of the finite element modeling and the VPS properties evaluation, VPS techniques were developed to produce a tungsten armored medium scale mockup using the optimum VPS tungsten-copper functional gradient joint.

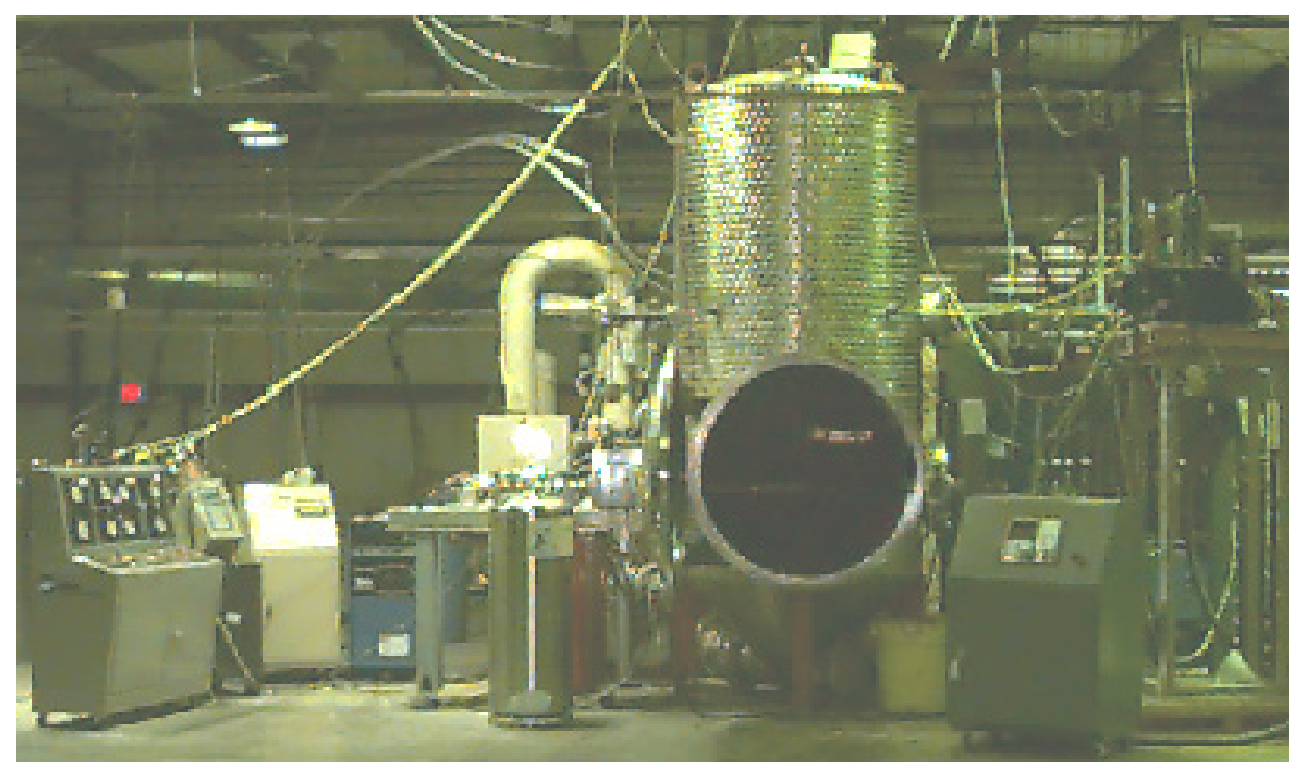

Figure 3 - One of three VPS chambers at PPI. 


\subsection{MODELING OF COPPER-TUNGSTEN FUNCTIONAL GRADIENT JOINT}

Previous numerical studies by Richard Nygren at Sandia National Laboratories [1,2] revealed a reduction in stresses using a $1 \mathrm{~mm}$ interlayer of soft copper (OFHC) compared with a sharp interface. Stresses were also obtained for a W/CuCrZr sharp interface and for a $1 \mathrm{~mm}$ $50 \% \mathrm{Cu} / 50 \% \mathrm{~W}$ composite interlayer. Nygren obtained lower stresses using the soft copper interlayer compared with the latter two cases. Accumulation of plastic strain was observed by applying and removing the thermal load several times, which suggest that thermal fatigue may be of concern using this soft $\mathrm{OFHC} \mathrm{Cu}$ interlayer.

For this study, a sharp $\mathrm{CuCrZr} / \mathrm{W}$ joint and different architectures of a layer between these two materials were modeled using ABAQUS, a general purpose finite element code. Soft oxygenfree high conductivity $(\mathrm{OFCH})$ copper and several compositionally graded materials of this high purity copper and tungsten were used as an interlayer. Plasticity, strain hardening and properties temperature dependence was considered.

The effect of changing the interlayer composition and thickness was investigated, stress and strain comparisons for the different architectures are presented, and optimization of the interlayer based on reduction of these values is suggested.

\subsection{Finite Element Model}

The geometry analyzed is a divertor mock up shown in Figure 4 which is used to remove heat and impurities from a fusion reaction chamber. This divertor is formed by three sections of different materials. At the top, the $\mathrm{W}$ armor faces the plasma and limits the radiation damage to the next section. Joined to the $\mathrm{W}$ armor, the $\mathrm{CuCrZr}$ heat sink removes heat using water flowing through a channel in the middle of this component. The heat sink is seated in a 316 stainless steel back piece. All the components have a width of $16 \mathrm{~mm}$, the armor is $5 \mathrm{~mm}$ long, the heat sink is $16 \mathrm{~mm}$ and the back is $20 \mathrm{~mm}$. The same dimensions were used for a previous analysis by R.E. Nygren [1-3].

Two load cases are analyzed. First the entire piece is cooled down from $550{ }^{\circ} \mathrm{C}$ to $25{ }^{\circ} \mathrm{C}$ and the residual stresses from these fabrication conditions are obtained. The second step begins with the state of stress and strain previously obtained and simulates the operation of this piece with a load of $5 \mathrm{MW} / \mathrm{m}^{2}$ applied to the top of the $\mathrm{W}$ armor and water flowing through a channel in the $\mathrm{CuCrZr}$ at $160{ }^{\circ} \mathrm{C}, 3.8 \mathrm{MPa}$ and $10 \mathrm{~m} / \mathrm{s}$. Stress and strain fields are obtained from the resulting temperature profile.

To model the heat transfer from the $\mathrm{CuCrZr}$ to the channel, values of the convective heat transfer were considered as a function of temperature as shown in Table 1. 


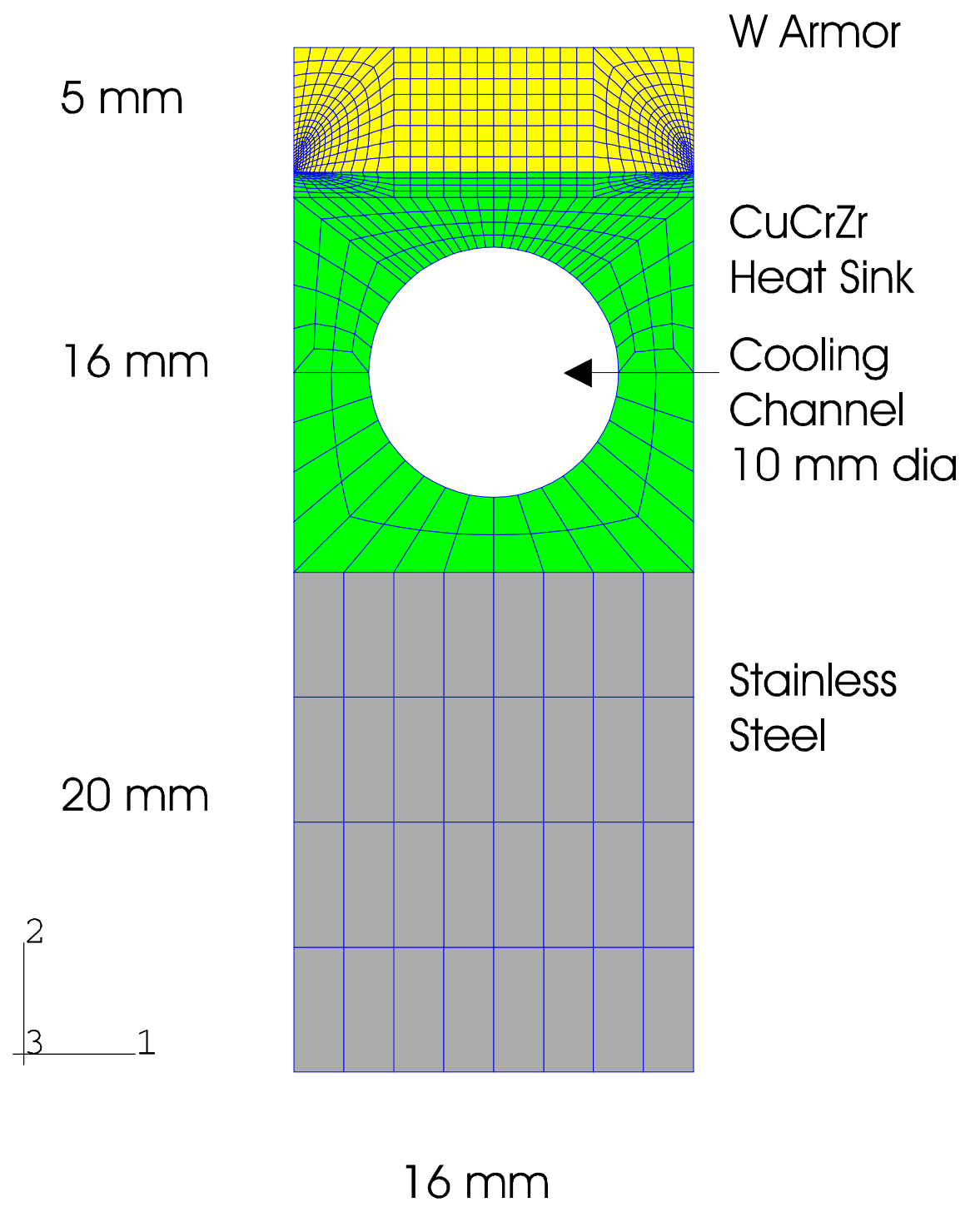

Figure 4 - Geometry of divertor. Sharp interface architecture. Half of the geometry modeled because to symmetry. 
Table 1 - Convection heat transfer coefficients vs temperature used in the channel.[3]

\begin{tabular}{|c|c|}
\hline $\mathbf{h}$ & $\mathbf{T}$ \\
$\mathbf{W} / \mathbf{c m}^{2} /{ }^{\circ} \mathbf{C}$ & ${ }^{\circ} \mathbf{C}$ \\
\hline 14.000 & 100.00 \\
14.105 & 160.00 \\
14.357 & 200.19 \\
14.461 & 223.89 \\
14.560 & 255.24 \\
15.153 & 278.69 \\
18.201 & 302.13 \\
23.026 & 320.58 \\
24.590 & 324.95 \\
25.123 & 326.35 \\
26.750 & 330.41 \\
28.979 & 335.54 \\
31.268 & 340.38 \\
33.609 & 344.98 \\
34.798 & 347.20 \\
\hline
\end{tabular}

The ABAQUS finite element code is used for the present model. Ten node biquadratic generalized plane strain elements (CGPE10R) of $0.05 \mathrm{~mm}$ size were used around the area where the joint of the $\mathrm{W}$ and $\mathrm{CuCrZr}$ reaches the free surface. For the case where the $\mathrm{W}$ is joined directly to the $\mathrm{CuCrZr}$, a second model with $0.025 \mathrm{~mm}$ element size was constructed in order to check for convergence. Eight node quadratic (DC2D8) diffusive heat transfer elements were used for the heat transfer analysis. The mesh is refined around the area where the $\mathrm{W} / \mathrm{CuCrZr}$ interface reaches the free surface because of the stress singularity that occurs in the free edge of a bimaterial interface zone.

The logic of the present analysis is as follows. First a heat transfer analysis is performed to determine the temperature distribution in steady state at the conditions of operation previously specified. Second a stress analysis is performed, this is divided in two steps. In the first step all the nodes are at initial temperature of $550{ }^{\circ} \mathrm{C}$ and the temperature is lowered to $25^{\circ} \mathrm{C}$. Residual stresses and strains are obtained from this first step. In the second step the temperature profile obtained in the heat transfer analysis is applied to the mesh. Thermal stresses and strains are obtained from this step.

The state of stress and strain of the first step is used as initial condition for the second step. Plastic strain is accumulated from one step to the other.

Only half of the piece is modeled due to symmetry on geometry and loads. Boundary conditions constraining the horizontal displacement of the nodes along the vertical centerline of the piece were applied. Furthermore, the vertical displacement was constrained in the first node at the bottom of the centerline. 
An additional model without the channel was performed in order to observe the change in stresses at the interface and determine if the results of the present study can be generalized to similar systems without the channel.

Next is a description of the different joint architectures analyzed:

- Sharp Interface.

- OFHC $\mathrm{Cu}$ layer. $\mathrm{W}$ and $\mathrm{CuCrZr}$ are joined by a $1 \mathrm{~mm}$ thick soft interlayer of high purity OFHC copper.

- Two graded linear interlayer. $1 \mathrm{~mm}$ thick layer composed by two $0.5 \mathrm{~mm}$ interlayers of the next compositions: $66 \% \mathrm{~W} / 33 \% \mathrm{OFHC}, 33 \% \mathrm{~W} / 66 \% \mathrm{OFHC}$.

- Four graded linear interlayer. $1 \mathrm{~mm}$ layer composed by four $0.25 \mathrm{~mm}$ interlayers of the next compositions: $\quad 80 \% \mathrm{~W} / 20 \% \mathrm{OFHC}, \quad 60 \% \mathrm{~W} / 40 \% \mathrm{OFHC}, \quad 40 \% \mathrm{~W} / 60 \% \mathrm{OFHC}$, $20 \% \mathrm{~W} / 80 \% \mathrm{OFHC}$.

- Four graded $\mathrm{Cu}$ rich. $1 \mathrm{~mm}$ layer composed by four $0.25 \mathrm{~mm}$ interlayers with the following compositions. $\quad 80 \% \mathrm{~W} / 20 \% \mathrm{OFHC}, \quad 60 \% \mathrm{~W} / 40 \% \mathrm{OFHC}, \quad 30 \% \mathrm{~W} / 70 \% \mathrm{OFHC}$, $10 \% \mathrm{~W} / 90 \% \mathrm{OFHC}$.

- Four graded W rich. Same thickness but following compositions: $96 \% \mathrm{~W} / 4 \% \mathrm{OFHC}$, $77 \% \mathrm{~W} / 23 \% \mathrm{OFHC}, 51 \% \mathrm{~W} / 49 \% \mathrm{OFHC}, 18 \% \mathrm{~W} / 82 \% \mathrm{OFHC}$.

- Four graded $\mathrm{Cu}$ rich. $2 \mathrm{~mm}$ interlayer. Four $0.5 \mathrm{~mm}$ interlayers of the next compositions: $80 \% \mathrm{~W} / 20 \% \mathrm{OFHC}, 60 \% \mathrm{~W} / 40 \% \mathrm{OFHC}, 40 \% \mathrm{~W} / 60 \% \mathrm{OFHC}, 20 \% \mathrm{~W} / 80 \% \mathrm{OFHC}$.

- Four graded $\mathrm{Cu}$ rich. $4 \mathrm{~mm}$ interlayer. Same compositions than for 1 and $2 \mathrm{~mm} \mathrm{Cu}$ rich.

The following six meshes were created:

1. Sharp interface coarse

2. Sharp interface refined

3. Sharp interface without channel

4. $1 \mathrm{~mm}$ interlayer

5. $2 \mathrm{~mm}$ interlayer

6. $4 \mathrm{~mm}$ interlayer

The element sizes on the sharp interface "coarse" mesh are of similar size to those used in the 1 $\mathrm{mm}, 2 \mathrm{~mm}$ and $4 \mathrm{~mm}$ meshes. The "refined" and no channel meshes were used to observe the sensibility of the results to these changes.

All the different compositions of the interlayer were modeled using the $1 \mathrm{~mm}$ interlayer mesh. The $1 \mathrm{~mm}$ interlayer was divided in four sets of sub-layers of $0.25 \mathrm{~mm}$ each. Changing the material properties of each of these sub-layers the different interlayer compositions were modeled. This is schematically shown in Figure 5. The optimum graded composition obtained for the $1 \mathrm{~mm}$ interlayer was used on the $2 \mathrm{~mm}$ and $4 \mathrm{~mm}$ interlayers.

Generalized plane strain elements are used in this analysis, this kind of element lies between two planes, that can move as rigid bodies with respect to each other allowing thermal strain in the out of plane direction. This kind of element is recommended [4,5] for 2D thermal stress analysis. A schematic representation of a generalized plane strain element is shown in Figure 6. 


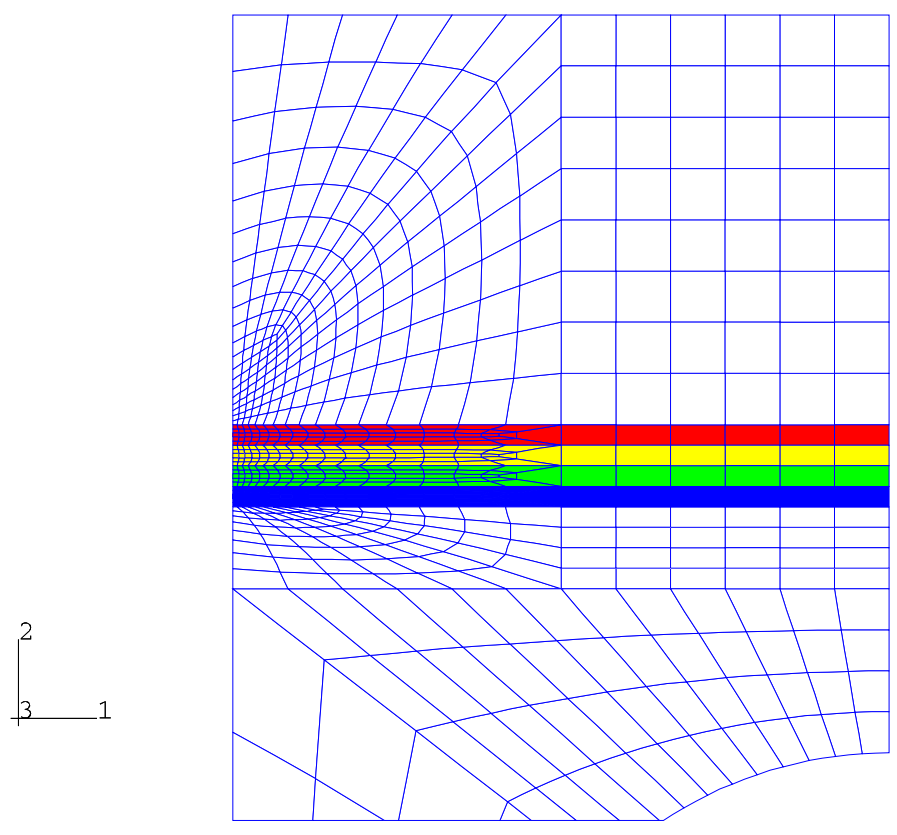

\begin{tabular}{|c|c|c|c|c|}
\hline $\mathrm{OFHC}$ & $\begin{array}{l}2 \text { layer } \\
\text { linear }\end{array}$ & $\begin{array}{l}4 \text { layer } \\
\text { linear }\end{array}$ & $\begin{array}{l}4 \text { layer } \\
\text { Cu rich }\end{array}$ & $\begin{array}{l}4 \text { layer } \\
\text { W rich }\end{array}$ \\
\hline $0 \%$ & $\begin{array}{l}33 \% \mathrm{Cu} \\
66 \% \mathrm{Cu}\end{array}$ & $\begin{array}{l}20 \% \mathrm{Cu} \\
40 \% \mathrm{Cu} \\
60 \% \mathrm{Cu} \\
80 \% \mathrm{Cu}\end{array}$ & $\begin{array}{l}20 \% \mathrm{Cu} \\
40 \% \mathrm{Cu} \\
70 \% \mathrm{Cu} \\
90 \% \mathrm{Cu}\end{array}$ & $\begin{array}{l}4 \% \mathrm{Cu} \\
23 \% \mathrm{Cu} \\
49 \% \mathrm{Cu} \\
82 \% \mathrm{Cu}\end{array}$ \\
\hline
\end{tabular}

Figure 5 - $1 \mathrm{~mm}$ layer architecture. 


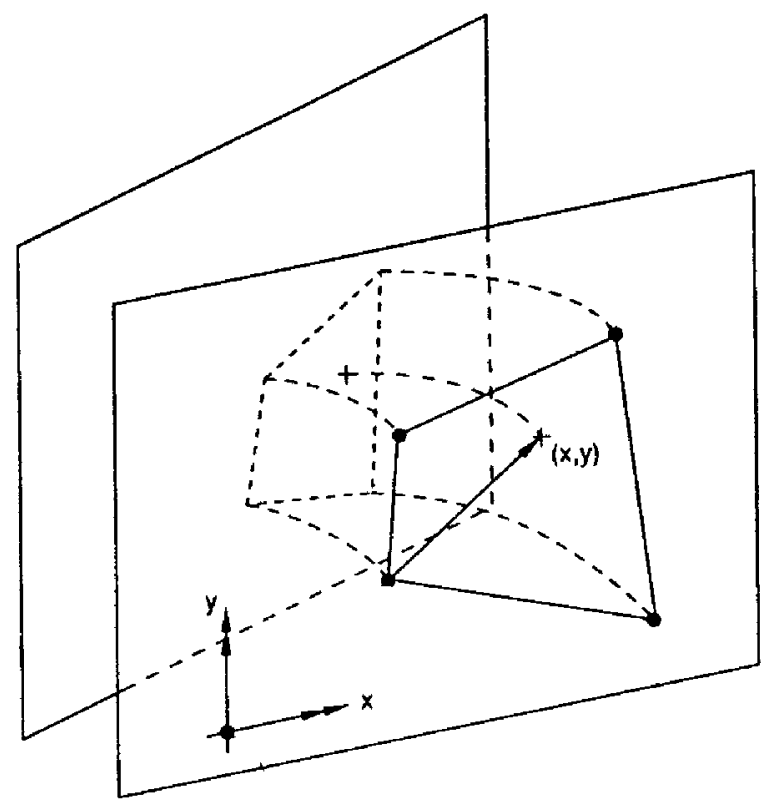

Figure 6 - Schematic representation of a generalized plane strain element. [4,5] 


\subsection{Material Properties}

The properties of $\mathrm{W}, \mathrm{CuCrZr}$ alloy (Elbrodur G) and 316 stainless steel were obtained from the literature [3,6,7]. In order to estimate the elastic and plastic properties of the different composites used as interlayer a modified rule of mixtures (ROM) was used. This ROM works well for functionally graded materials [8-10]. Figure 7 shows a schematic representation of this ROM. In this rule of mixtures the composite is considered isotropic, the stress-strain curve of the composite is proportional to the volume fraction of both constituents. A stress-strain transfer coefficient $q$ is defined, which is the slope of the line that joins the stress-strain curves of the two constituents of the composite.

$$
\begin{aligned}
& \sigma_{c}=\sigma_{\alpha} V_{\alpha}+\sigma_{\alpha} V_{\alpha} \\
& \varepsilon_{c}=\varepsilon_{\alpha} V_{\alpha}+\varepsilon_{\beta} V_{\beta} \\
& q=\frac{\sigma_{\alpha}-\sigma_{\beta}}{\varepsilon_{\alpha}-\varepsilon_{\beta}}
\end{aligned}
$$

The value of q used in the current model, $-4.5 \mathrm{GPa}$, was based on earlier work [8,9] for the elastic-plastic system $\mathrm{Al}_{2} \mathrm{O}_{3}-\mathrm{Ni}$. Williamson et al showed that the stress is not very sensitive to changes in the transfer coefficient [8]. $\mathrm{q}=0$ and $\mathrm{q}=\infty$ are the extremes indicating iso-stress and iso-strain conditions respectively. Suresh [11] shows that combining the equations (1), (2), (3) and invoking Hooke's law $(\sigma=\mathrm{E} \varepsilon)$ we obtain:

$$
\begin{aligned}
& E_{c}=\left[V_{C u}\left(\frac{q+E_{W}}{q+E_{C u}}\right)+V_{W}\right]^{-1}\left[V_{C u} E_{C u}\left(\frac{q+E_{W}}{q+E_{C u}}\right)+V_{W} E_{W}\right] \\
& \sigma_{y c}=\sigma_{y C u}\left[V_{C u}+\left(\frac{q+E_{C u}}{q+E_{W}}\right) \frac{E_{W}}{E_{C u}} V_{W}\right] \\
& H_{c}=\left[V_{C u}\left(\frac{q+E_{W}}{q+H_{C u}}\right)+V_{W}\right]^{-1}\left[V_{C u} H_{C u}\left(\frac{q+E_{W}}{q+H_{C u}}\right)+V_{W} E_{W}\right]
\end{aligned}
$$

where $\mathrm{H}$ is the elastoplastic tangent modulus describing the strain hardening of the different materials. $\mathrm{E}_{\mathrm{W}}$ and $\mathrm{E}_{\mathrm{Cu}}$ are the elastic modulus of tungsten and copper respectively.

The thermal expansion coefficient was obtained using the Turner equation where the CTE is function of the volume fraction of the present constituents and the bulk modulus of these. This equation is as follows: 
$\alpha_{c}=\frac{\alpha_{C u} V_{C u} K_{C u}+\alpha_{W} V_{W} K_{W}}{V_{C u} K_{C u}+V_{W} K_{W}}$

where $\mathrm{K}$ is the bulk modulus of the correspondent phases. This expression assumes that no cracks develop, equal contraction exists at all the grains, and pure hydrostatic stresses contribution, neglecting deviatoric stresses induced by shear at the interfaces $[12,13]$.

For the thermal conductivity, blocks of the two constituents arranged parallel or perpendicular to the heat flow are defined as the maximum and minimum bounds. In the current analysis the perpendicular arrangement was used because it is the most conservative. Its conservative nature arise because of the lower heat conductivity obtained which is reflected in the higher temperature difference between top and channel resulting in larger stress and strain fields.

All other properties required for this calculation were obtained by a linear rule of mixtures based on the volume fraction of the different constituents. This ROM is defined as:

$P_{c}=V_{C u} P_{C u}+V_{W} P_{W}$

where $\mathrm{P}$ is the material property and $\mathrm{V}_{\mathrm{i}}$ is the volume fraction of the material $\mathrm{i}$.

Plasticity was considered and isotropic strain hardening was defined for the different materials as a straight line after yield. To define this in ABAQUS, a value for the true yield stress $\sigma_{\mathrm{o}}$ at $0 \%$ of plastic strain $\varepsilon^{\mathrm{pl}}$ and a second yield stress $\sigma_{02}$ at another value of plastic strain were specified. Temperature dependent thermal expansion coefficient, conductivity, elastic modulus, yield strength and elastoplastic tangent modulus were considered [3,6,7]. For the different graded materials these relations of properties and temperature were obtained by using the previously described ROM's.

For the $\mathrm{Cu}$ rich and $\mathrm{W}$ rich graded interlayers the following power law equation previously applied by Drake et al. and Torres et al. [9,14] was used:

$$
V_{C u}=\left(\frac{x}{t}\right)^{p}
$$

where $\mathrm{x}$ is the distance from the $\mathrm{W}$ to the position in thickness, $\mathrm{t}$ is the total thickness of the graded region and $\mathrm{p}$ is an arbitrary exponent. Discrete layers are used to approximate the gradient. Values of $\mathrm{p}$ of 0.75 and 1.5 were used for the $\mathrm{Cu}$ rich and $\mathrm{W}$ rich graded interlayers respectively. Figure 8 shows the composition gradients for different values of $p$.

Based on the volume fractions, the modified rule of mixtures was used obtaining the properties of each interlayer. The properties of the different materials used for this analysis at $20^{\circ} \mathrm{C}$ are presented in Table 2 . 


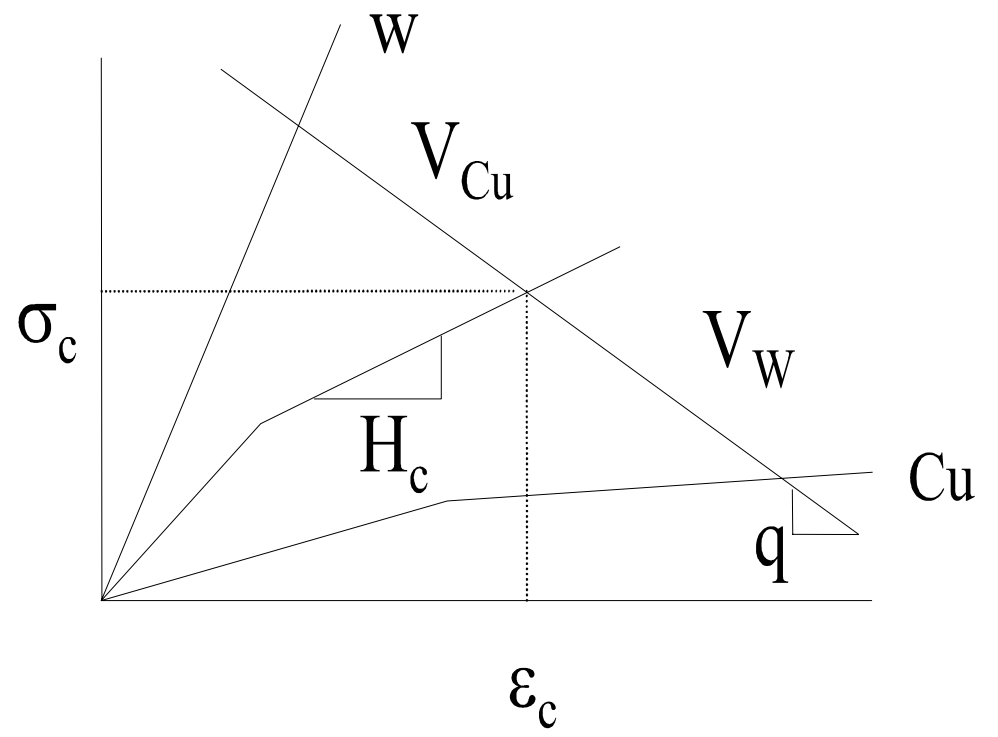

Figure 7 - Scheme of the modified rule of mixtures.

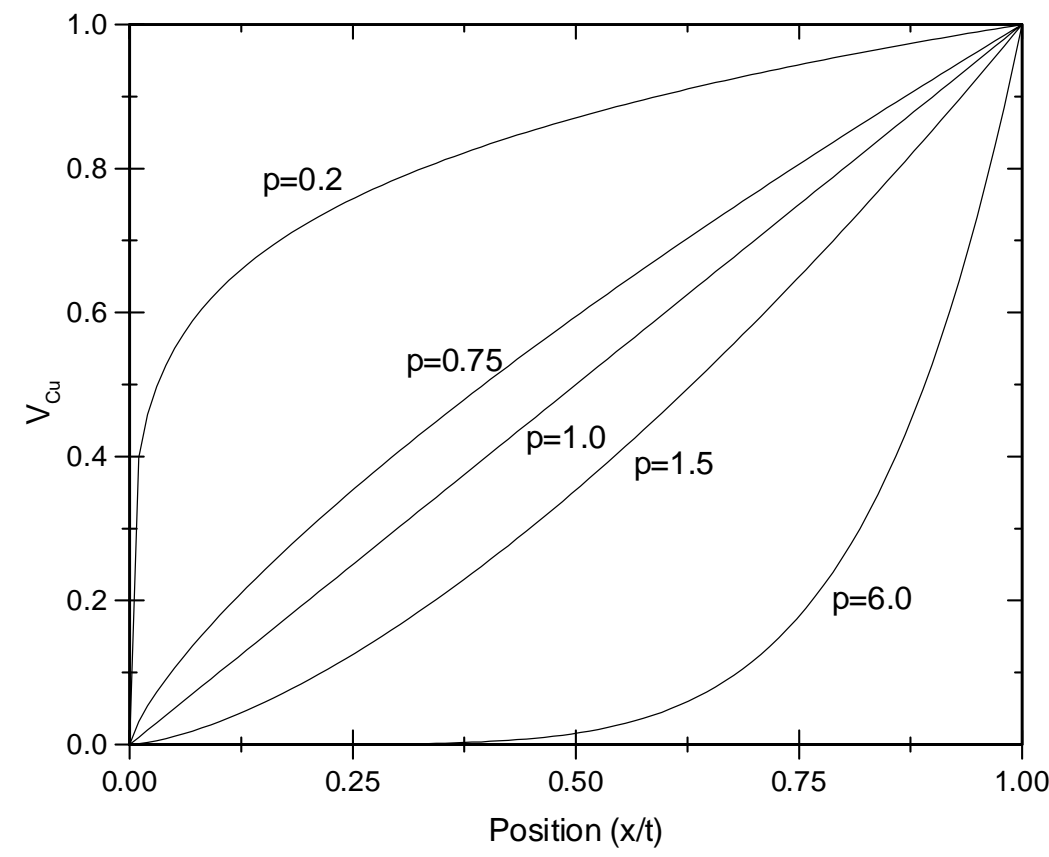

Figure 8 - Volume fraction of $\mathrm{Cu}$ for different values of the $\mathrm{p}$ exponent. 
Table 2 - Material properties at room temperature, $\mathrm{q}=-4.5 \mathrm{GPa}$.

\begin{tabular}{|c|c|c|c|c|c|c|c|c|}
\hline & E & $v$ & $\overline{\sigma 0}$ & $\mathrm{H}$ & CTE & k & C & $\rho$ \\
\hline Material & MPa & & MPa & Mpa & $1 / \mathrm{C}$ & W/cm-C & $\mathrm{J} / \mathrm{g}-\mathrm{C}$ & $\mathrm{Mg} / \mathrm{m}^{\wedge} 3$ \\
\hline$\overline{\mathbf{W}}$ & $\overline{05000}$ & $\overline{0.3}$ & 437.00 & 334.70 & $.67 \mathrm{E}-06$ & 1.767 & 0.110 & 19.10 \\
\hline Elbrodur & 9310 & 3 & 49.00 & 1227.00 & $6 \mathrm{E}$ & 38 & 384 & $\overline{8.8}$ \\
\hline 316 SS & 4000 & 0.3 & 274.00 & & $1.59 \mathrm{E}-05$ & 0.139 & 0.465 & 7.9 \\
\hline OFHC & 124000 & 0.3 & 42.00 & 840.91 & $1.66 \mathrm{E}-05$ & 4.000 & 0.430 & 8.90 \\
\hline $4 \% \mathrm{Cu}$ & 368886 & 0.3 & 42.96 & 97178.50 & $4.84 \mathrm{E}-06$ & 1.699 & 0.124 & 18.65 \\
\hline $20 \% \mathrm{CU}$ & 280315 & 0.3 & 42.79 & 20879.80 & $5.53 \mathrm{E}-06$ & 1.874 & 0.174 & 17.06 \\
\hline $23 \% \mathrm{Cu}$ & 267889 & 0.3 & 42.76 & 17749.30 & $5.68 \mathrm{E}-06$ & 1.912 & 0.184 & 16.75 \\
\hline $33 \% \mathrm{Cu}$ & 232404 & 0.3 & 42.66 & 11266.80 & $6.27 \mathrm{E}-06$ & 2.055 & 0.217 & 15.70 \\
\hline $40 \% \mathrm{Cu}$ & 213897 & 0.3 & 2.59 & 8595.93 & $6.71 \mathrm{E}-06$ & 2.160 & 0.238 & 15.02 \\
\hline $49 \% C$ & 192325 & 0.3 & 2.49 & 6253.76 & $7.44 \mathrm{E}-06$ & 2.328 & 0.268 & 14.06 \\
\hline 60\% & 25 & 0.3 & 38 & 4324.74 & $8.46 \mathrm{E}-06$ & 2.550 & 0.302 & 12.98 \\
\hline & 21 & 0.3 & .31 & 3538.25 & $9.23 \mathrm{E}-06$ & 2.711 & 0.323 & 12.31 \\
\hline $70 \%$ & 156882 & 0.3 & 27 & 3087.44 & $9.73 \mathrm{E}-06$ & 2.811 & 0.335 & 11.93 \\
\hline & 4435 & 0.3 & 17 & 2154.43 & 1.13E-05 & 3.110 & 0.366 & 10.94 \\
\hline $82 \%$ & 2334 & 0.3 & 42.15 & & 1.1 & 3.173 & 0.372 & 10.76 \\
\hline $90 \% \mathrm{Cu}$ & 13295 & 0.3 & 42.06 & 1425.75 & $1.36 \mathrm{E}-05$ & 3.516 & 0.400 & 9.8 \\
\hline
\end{tabular}

\section{$2.3 \quad$ Results}

\section{$\underline{\text { Notation }}$}

Variables are presented in ABAQUS format. Table 3 shows the different variables used in this document, the nomenclature used here and other forms to represent them usually found in literature. The double numeric subscript is used in components of stress, strain or other tensor variables. Subscripts 1, 2 and 3 refer to the directions in the orthogonal coordinate system in which the model is based.

Table 3 - Variables used in this analysis.

\begin{tabular}{|c|c|l|}
\hline ABAQUS Variable & Literature & Description \\
\hline S11 & $\sigma_{\mathrm{x}}$ & Stress in $x$ or in plane 1 direction 1. \\
\hline S22 & $\sigma_{\mathrm{y}}$ & Stress in y or in plane 2 direction 2. \\
\hline S33 & $\sigma_{\mathrm{z}}$ & Stress in z or in plane 3 direction 3. \\
\hline S12 & $\tau_{\mathrm{xy}}$ & Shear stress in plane 1 with direction 2. \\
\hline MISES & $\sigma$ & Effective or equivalent Mises stress. \\
\hline PRESS & $\sigma_{\mathrm{m}}$ & Hydrostatic stress or mean stress. \\
\hline PEEQ & $\varepsilon$ & Effective mises plastic strain. \\
\hline
\end{tabular}


To identify the different variables and relate them to the respective architecture, a shorthand notation of four fields is used in the plots. This is illustrated with the following example:

\section{SHS11I1}

where:
SH Sharp interface architecture
S11 Stress component
I Along the $\mathrm{W} /$ joint interface
1 Analysis step number 1

Table 4 shows the different values that each of the fields can take. The different locations where the plots are generated are shown in Figure 9.

Table 4 - Shorthand notation used in plots.

\begin{tabular}{|l|l|l|l|l|l|c|c|}
\hline Field 1 & Architecture & Field 2 & Variable & Field 3 & Location & Field 4 & Step \\
\hline SH & Sharp interface & S11 & S11 & I & W/joint interface & 1 & 1 \\
\hline OF & OFHC 1 mm & S22 & S22 & L & Left surface & 2 & 2 \\
\hline 2L & 2 layered 1mm linear & S12 & S12 & R & Centerline & & \\
\hline 4l & 4 layered 1 mm linear & PEQ & PEEQ & & & & \\
\hline 4C & 4 layered 1 mm Cu rich & & & & & & \\
\hline 4W & 4 layered 1 mm W rich & & & & & & \\
\hline 2M & 4 layered 2 mm Cu rich & & & & & & \\
\hline 4M & 4 layered 4 mm Cu rich & & & & & & \\
\hline
\end{tabular}

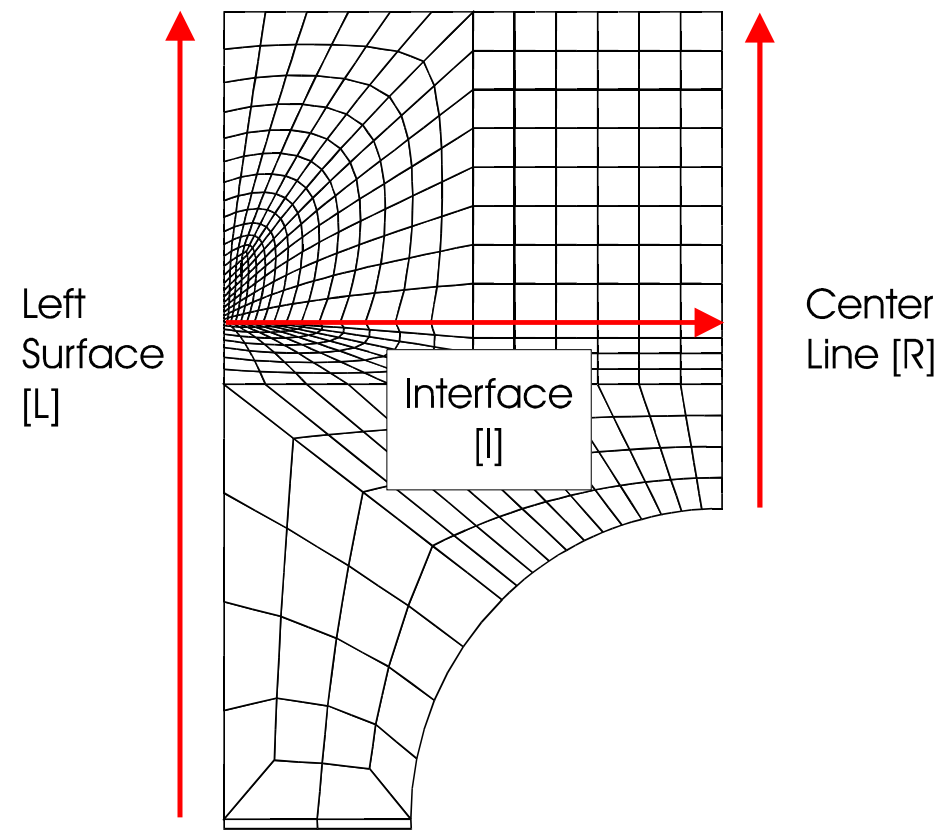

Figure 9 - Locations for x-y plots. 
The plastic strain is reported in terms of the effective or equivalent plastic strain (PEEQ variable for ABAQUS), the correspondent stress, is the equivalent stress (MISES in ABAQUS). These are invariants corresponding to the axial normal component stress and strain in a tension test [15]. In terms of the principal directions these are defined as:

$$
\begin{gathered}
\sigma_{e q}=\frac{\sqrt{2}}{2}\left[\left(\sigma_{1}-\sigma_{2}\right)^{2}+\left(\sigma_{2}-\sigma_{3}\right)^{2}+\left(\sigma_{3}-\sigma_{1}\right)^{2}\right]^{1 / 2} \\
\varepsilon_{e q}^{p l}=\frac{\sqrt{2}}{2}\left[\left(\varepsilon_{1}^{p l}-\varepsilon_{2}^{p l}\right)^{2}+\left(\varepsilon_{2}^{p l}-\varepsilon_{3}^{p l}\right)^{2}+\left(\varepsilon_{3}^{p l}-\varepsilon_{1}^{p l}\right)^{2}\right]^{1 / 2}
\end{gathered}
$$

where the subindex 1,2 and 3 stand for the principal directions, and the superindex $\mathrm{pl}$ indicates plastic strain.

Temperature profile with heat load of $5 \mathrm{MW} / \mathrm{m}^{2}$

Applying the heat load at the top of the $\mathrm{W}$ armor and removing heat at the cooling channel results in a vertical gradient in temperature at the divertor. The obtained temperature gradient for the sharp interface is shown in Figure 10. An increase in temperature at the top of the W armor is observed when an interlayer is used to join the $\mathrm{W}$ and the $\mathrm{CuCrZr}$ alloy. The minimum temperature obtained is $434{ }^{\circ} \mathrm{C}$ for the sharp interface, $448{ }^{\circ} \mathrm{C}$ using the $1 \mathrm{~mm}$ OFHC Cu . A temperature around $460{ }^{\circ} \mathrm{C}$ for the other $1 \mathrm{~mm}$ interlayers, finally $484{ }^{\circ} \mathrm{C}$ and $548{ }^{\circ} \mathrm{C}$ for the 2 $\mathrm{mm}$ and $4 \mathrm{~mm}$ interlayer architectures respectively. Temperature profiles for all the architectures are shown in appendix A.
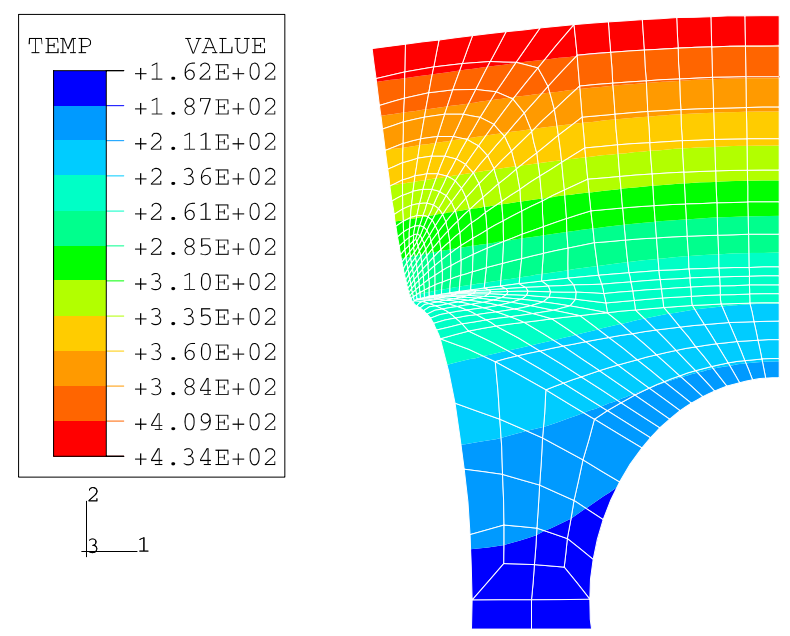

Figure 10 - Temperature distribution for heat load of $5 \mathrm{MW} / \mathrm{m} 2$ at the $\mathrm{W}$ top and heat removal by water flowing at $10 \mathrm{~m} / \mathrm{s}, 160{ }^{\circ} \mathrm{C}$ and $3.8 \mathrm{MPa}$ through the channel, for a sharp interface. Distortion amplified 25 times. 


\section{Singular corner problem}

S22 results around the area where the interface reaches the free surface must be evaluated with some reserve. Conventional solid elements at the corner can not reach exact solution even with the highest mesh refinement [1]. This problem arises from the traction free surface condition, which is not achieved by conventional solid elements used at the singular corner. The mesh has been highly refined around this area trying to minimize the possible error present. Even though S11 is not zero at the singular corner, the trend in almost all the models is that it approaches zero with exception of the soft copper interlayer and the heat load step of the $2 \mathrm{~mm}$ and $4 \mathrm{~mm}$ architectures. Figure 11 shows a comparison of the S11 residual stress for the different architectures.

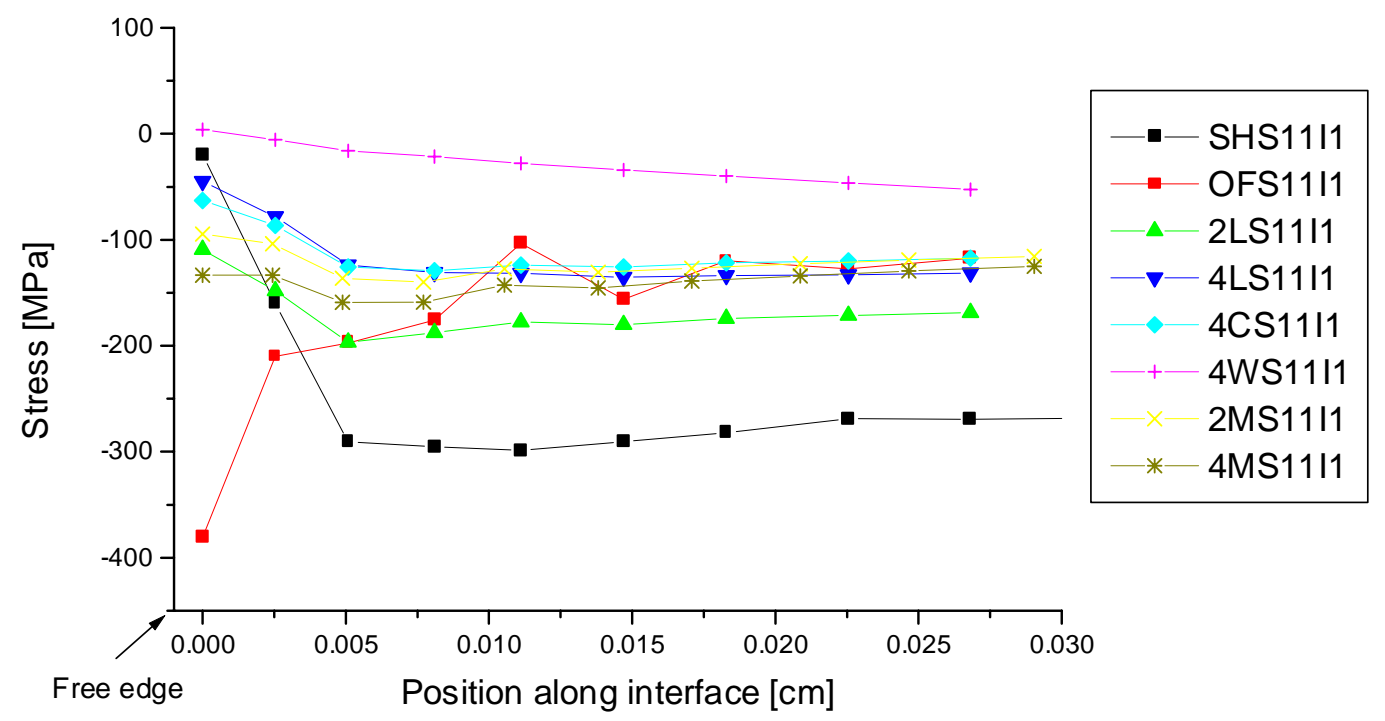

Figure 11 - Stress along the interface near the free edge for the different architectures.

It is known that failure, due to high S22 stress, is likely to occur at the singular corner area in a bimaterial interface $[8,16-18]$. Although some error is present in the solution around this area, the values obtained at this region are used on a comparative basis to try to understand the effect of the different interlayer architectures on stress and strain.

Two meshes were used to model the sharp interface. $0.05 \mathrm{~mm}$ and $0.025 \mathrm{~mm}$ elements were used around the singular corner for the "coarse" and "refined" mesh respectively. A comparison of residual stresses at the $\mathrm{W}$ armor along the joint for the two meshes is shown in Figure 12. S11 approaches zero at the free edge, and the maximum S22 value is just $1.2 \%$ higher for the refined mesh with a value of $497 \mathrm{MPa}$ vs. $503 \mathrm{MPa}$ on the coarse mesh.

Based on this comparison, $0.05 \mathrm{~mm}$ element size was used around the singular corner for the different architectures analyzed. 


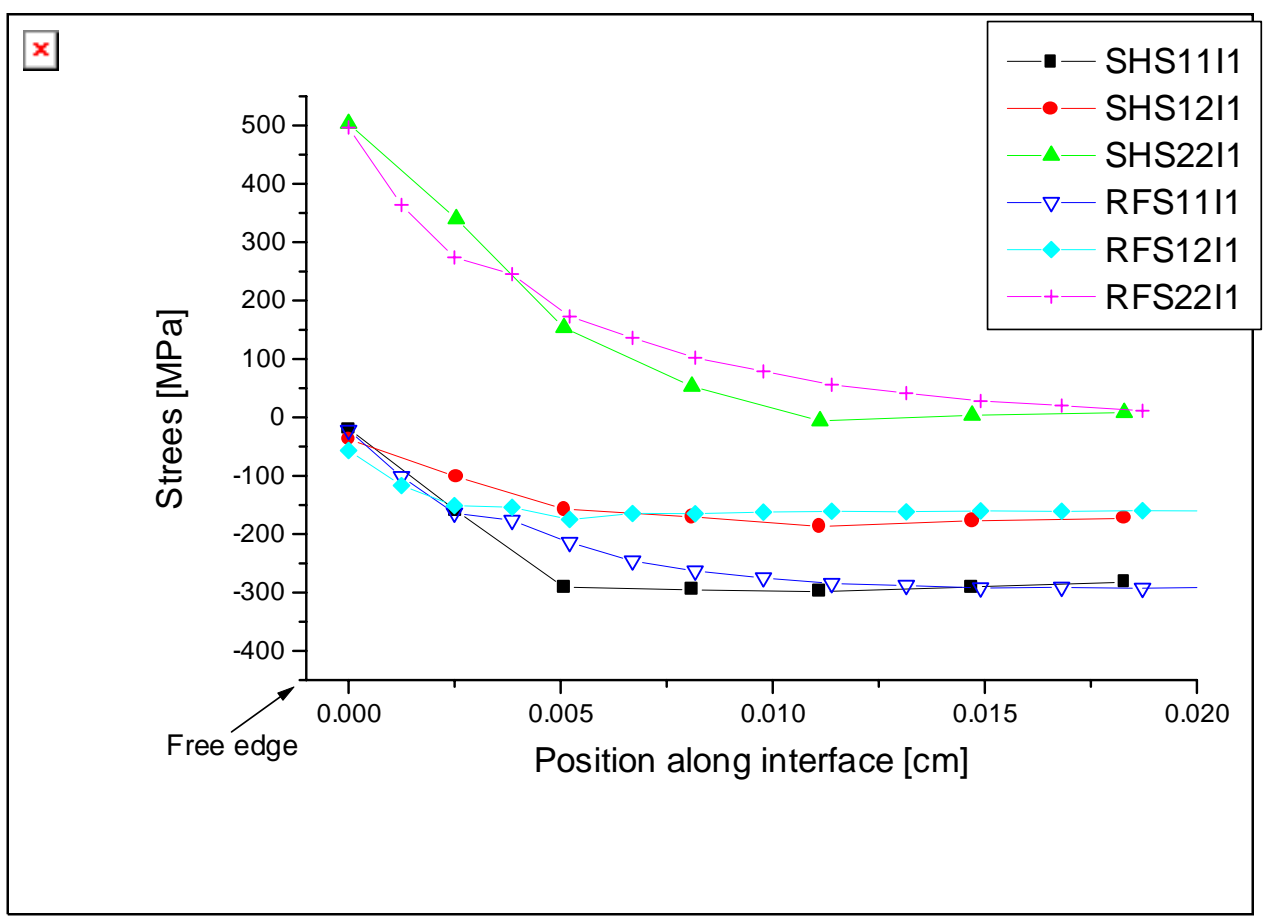

Figure 12 - Comparison of stresses along the interface near the free edge for "coarse" (SH) and "refined mesh" (RF).

\section{$\underline{\text { S11 Stress }}$}

Figure 13 shows a S11 contour plot of the distribution of S11 stress on the sharp interface, and 1 $\mathrm{mm} \mathrm{Cu}$ rich architectures for both steps. As in all the contour plots presented in this study, the distortion is amplified 25 times to illustrate bending. The S11 stress is higher at the top of the W armor for the sharp interface model. Because the higher S11 stresses are found at the centerline, S11 stresses are plotted in Figure 14 as a function of distance from the top of the cooling channel to the top of the tungsten armor. Stress results for both steps are plotted, after cooling down and after applying the heat load of $5 \mathrm{MW} / \mathrm{m}^{2}$ for the sharp interface architecture and $1 \mathrm{~mm} \mathrm{Cu}$ rich interlayer. The S11 stress component in this region is of concern due to the possible mode of failure of a crack with origin at the top of the $\mathrm{W}$ which then propagates perpendicular to the interface.

Residual S11 after cooling down is compressive as expected in the $\mathrm{W}$ next to the joint due to the lower CTE of this material. The stress gradually changes from compressive to tensile as we move vertically away from the joint to the top of the lower CTE W armor. After applying the heat load, the sign of the stress along the centerline changes. S11 becomes compressive at the top of the $\mathrm{W}$ and tensile next to the material to which it is joined.

The maximum S11 at the top of the tungsten is observed on the sharp interface architecture with a value of $271 \mathrm{MPa}$. A maximum reduction in the stress in this area is obtained using the OFHC $1 \mathrm{~mm}$ interlayer, with a value of $108 \mathrm{MPa}$ (Figure 13). The graded architecture showing the maximum reduction is the $2 \mathrm{~mm} \mathrm{Cu}$ rich interlayer with $173 \mathrm{MPa}$. The $1 \mathrm{~mm} \mathrm{Cu}$ rich shows a value of $180 \mathrm{MPa}$. 
As mentioned earlier, the temperature profile originated from the thermal load at the top of the armor and heat removal at the cooling channel, results in tensile stress at the $\mathrm{W}$ next to the joint and compressive stress at the top. The tensile stress at the $\mathrm{W}$ next to the joint is maximum for the OFHC architecture with a value of $236 \mathrm{MPa}$ and minimum for the $4 \mathrm{~mm} \mathrm{Cu}$ rich interlayer with $84 \mathrm{MPa}$.

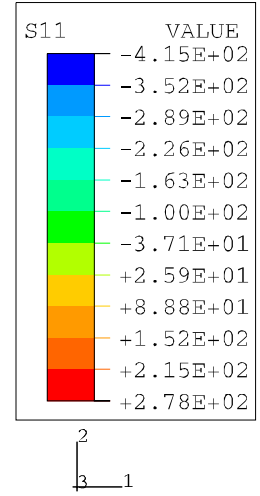

a)

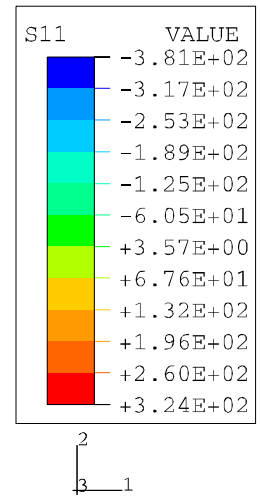

C)
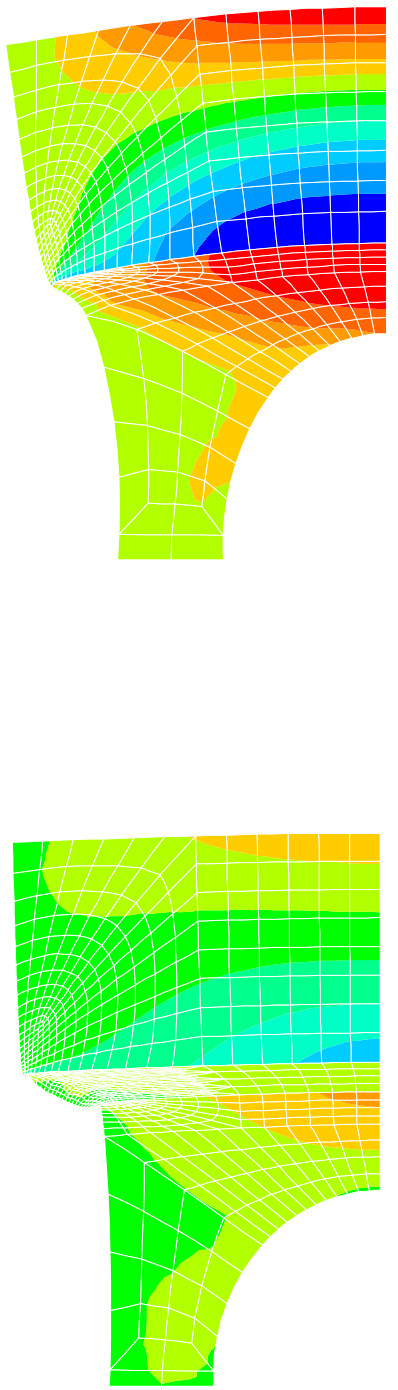

b)

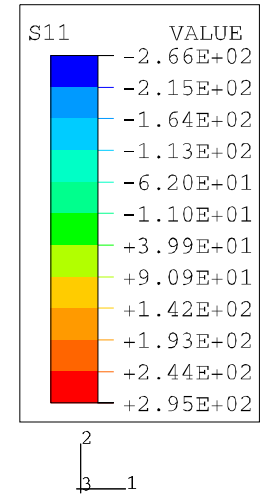

d)
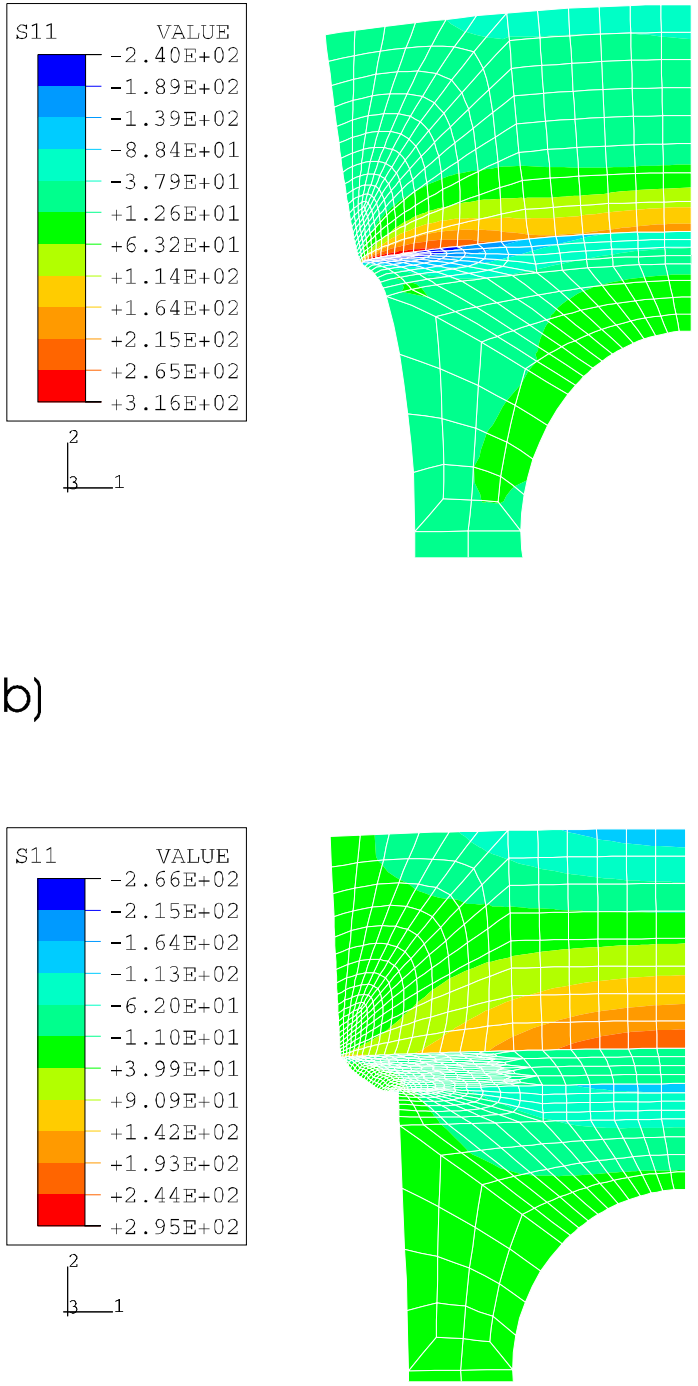

Figure 13 - S1 1 contour plots of:

sharp interface

a) after cooling down, b) heat load.

$1 \mathrm{~mm} \mathrm{Cu}$ rich

c) after cooling down, d) heat load.

Distortion factor $=25 \mathrm{X}$ 

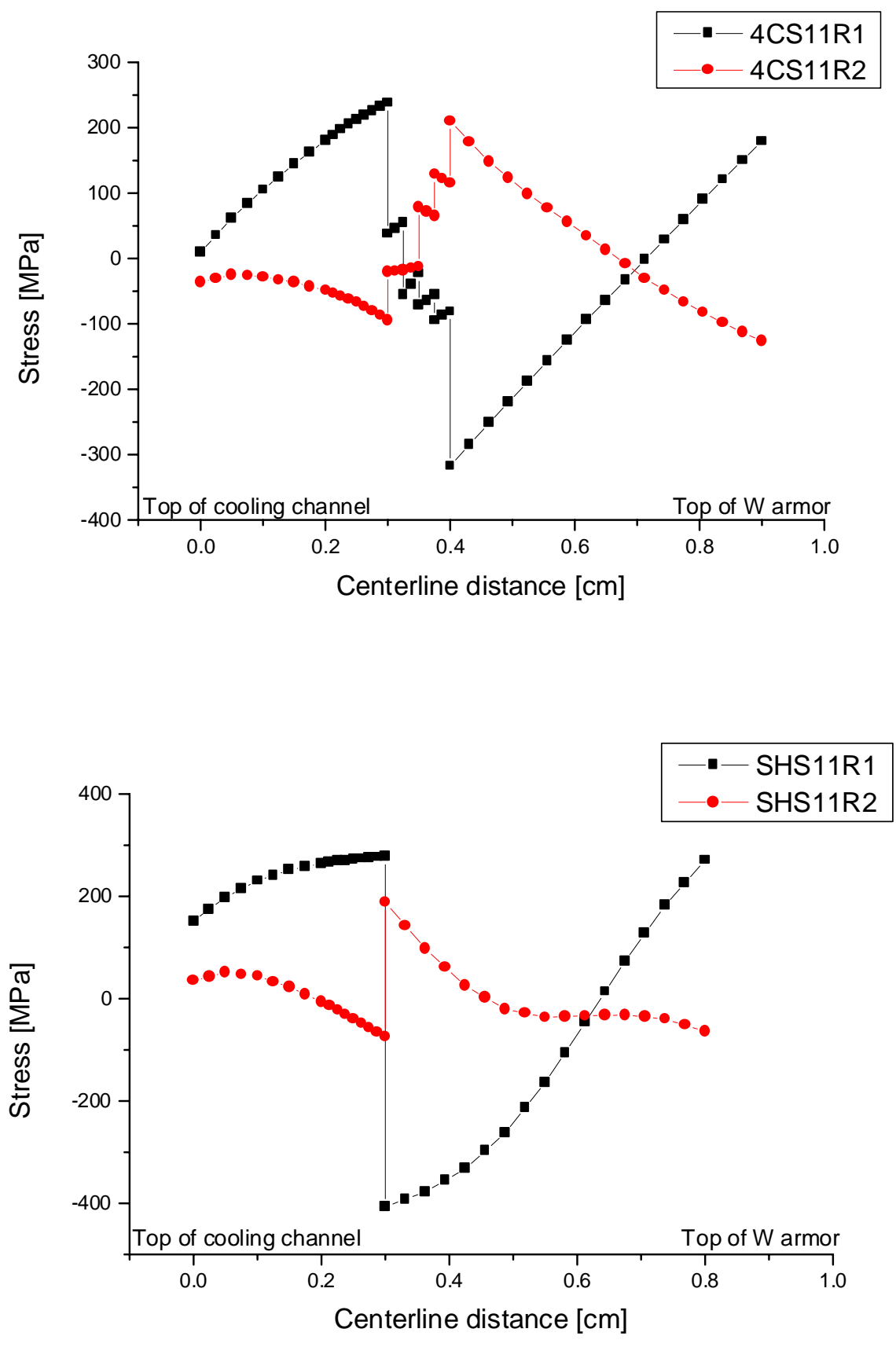

Figure 14 - S11 stress along centerline for a) sharp and b) $1 \mathrm{~mm} \mathrm{Cu}$ rich architectures after cooling down (square) and with heat load applied (circle). 


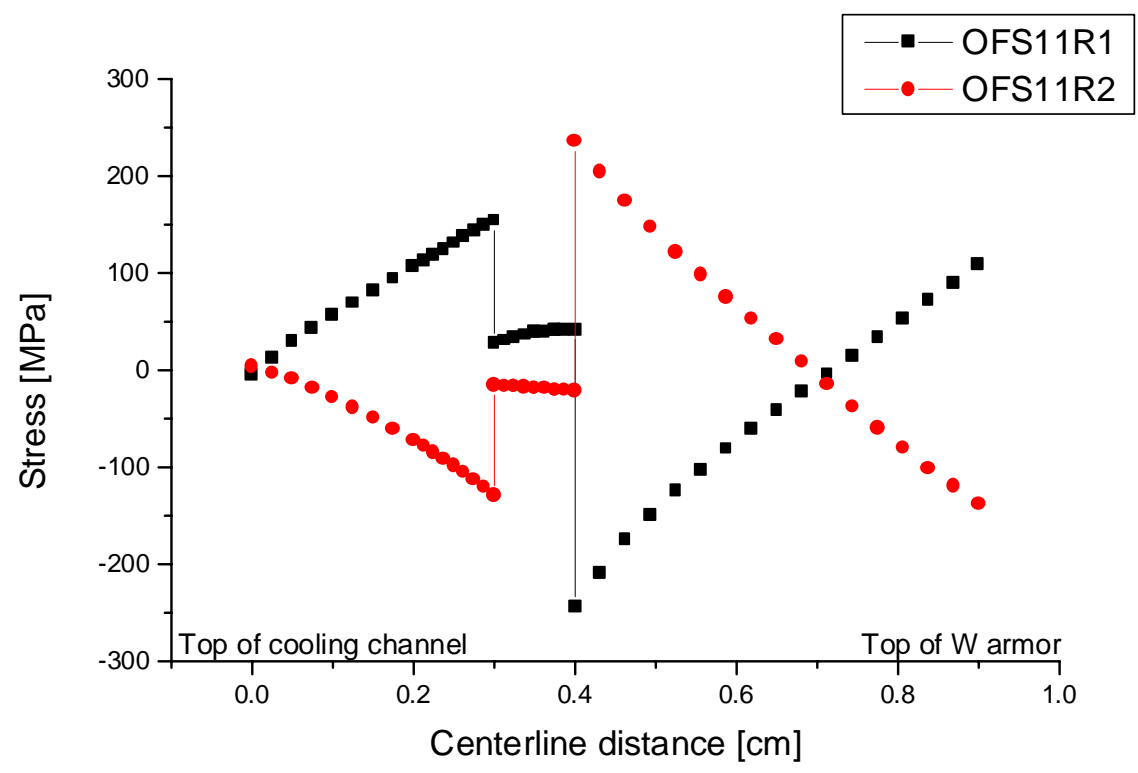

Figure 15 - S11 stress along centerline for the soft copper interlayer after cooling down (square) and with heat load applied (circle).

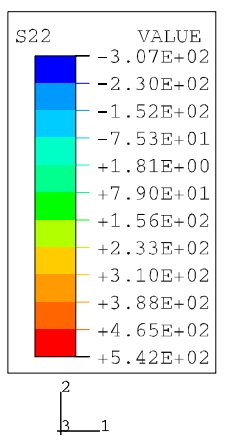

a)

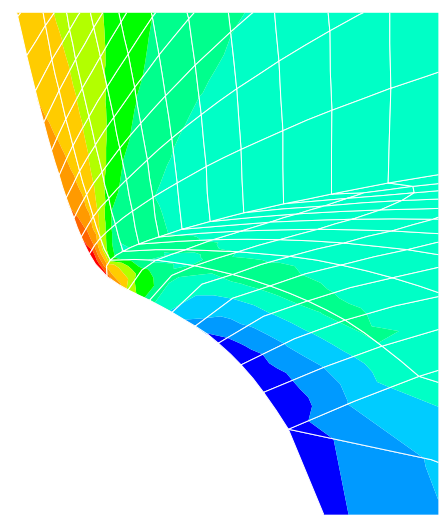

b)
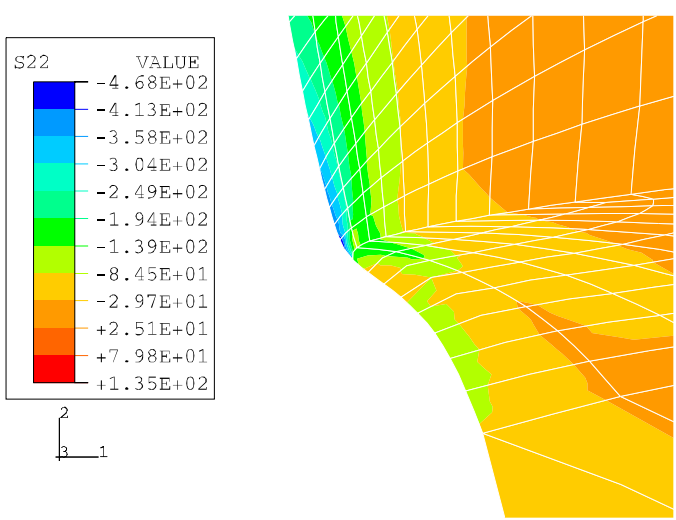

Figure 16 - S22 stress contour plots for the sharp interface architecture around the singular corner area. a) After cooling down, b) heat load applied. 
$\underline{\text { S22 stresses and plastic strain around the singular corner. }}$

A contour plot of the S22 stress for the sharp interface architecture is shown in Figure 16, distortion is amplified 25 times. Tensile stress is observed at the tungsten near the singular corner after cooling down. Applying the heat load results in compressive stress in this area. This behavior is observed using the different interlayers. Appendix A shows contour plots of S22 and other different variables for all the architectures.

The S22 stress and equivalent plastic strain along the left surface are plotted for the different architectures. Figure 17 shows S22 and PEEQ for the sharp interface. As shown in the contour plot, the stress at the $\mathrm{W}$ next to the interface and close to the free edge is tensile after cooling down and becomes compressive as a result of the heat load applied.

The maximum stress is for the sharp interface, with a value of $542 \mathrm{MPa}$. Using the soft copper (OFHC) interlayer resulted in the largest reduction in stress with $365 \mathrm{MPa}$ (Figure 18a). Figure $18 \mathrm{~b}$ shows the equivalent plastic strain along the left surface of this architecture. The OFHC next to the interface with $\mathrm{W}$ reaches a PEEQ of $12.4 \%$ after cooling down. Applying the heat load results in compressive S22 stress of $-357 \mathrm{MPa}$. A further increase in plastic strain to a value of $18.8 \%$ around this area is observed.

Using the 2 layered $1 \mathrm{~mm}$ linear material as an interlayer resulted in a maximum stress of 473 MPa after cooling down, as seen in Figure 19. The plastic strain for this architecture is maximum next to the $\mathrm{CuCrZr}$ interface and has a value of $4.3 \%$ after cooling down, increasing to $4.4 \%$ after applying the heat load. S22 and PEEQ for the 4 layered linear material are shown in Figure 20. The maximum S22 stress is $422 \mathrm{MPa}$ and a plastic strain of $7.6 \%$ is observed again at the interlayer next to the interface with the CuCrZr. Application of the heat load does not significantly alter the plastic strain distribution.

For the 4 layered $\mathrm{Cu}$ rich $1 \mathrm{~mm}$ interface (Figure 21), a stress of $390 \mathrm{MPa}$ is obtained and the plastic strain has a value of $10.4 \%$ again next to the joint with the $\mathrm{CuCrZr}$. Once again no change is observed in the plastic strain distribution after applying the heat load. Using the $\mathrm{W}$ rich interlayer gives a stress of $509 \mathrm{MPa}$ and plastic strain of $8 \%$ (Figure 22).

The effect of increasing the interlayer thickness to $2 \mathrm{~mm}$ but using 4 gradients with the same compositions as in the $1 \mathrm{~mm} \mathrm{Cu}$ rich case is shown in Figure 23. A stress of $372 \mathrm{MPa}$ is obtained for this architecture, the peak of equivalent plastic strain is reduced to $7.9 \%$, and no further accumulation of plastic strain after the heat load is observed. Using the same $\mathrm{Cu}$ rich composition gradient but thickness of $4 \mathrm{~mm}$ results also in a maximum stress of $372 \mathrm{MPa}$, as seen in Figure 24; the equivalent plastic strain peak is reduced to $5.6 \%$.

A reduction in S22 compressive stress at the $\mathrm{CuCrZr}$ next to the joint is observed as the thickness is increased. For the $1 \mathrm{~mm}$ case $-314 \mathrm{MPa}$ is obtained, $-294 \mathrm{MPa}$ for the $2 \mathrm{~mm}$, and $-250 \mathrm{MPa}$ for the $4 \mathrm{~mm}$. Figure 25 shows S22 contour plots for the $1 \mathrm{~mm}, 2 \mathrm{~mm}$ and $4 \mathrm{~mm} \mathrm{Cu}$ rich graded architectures. 

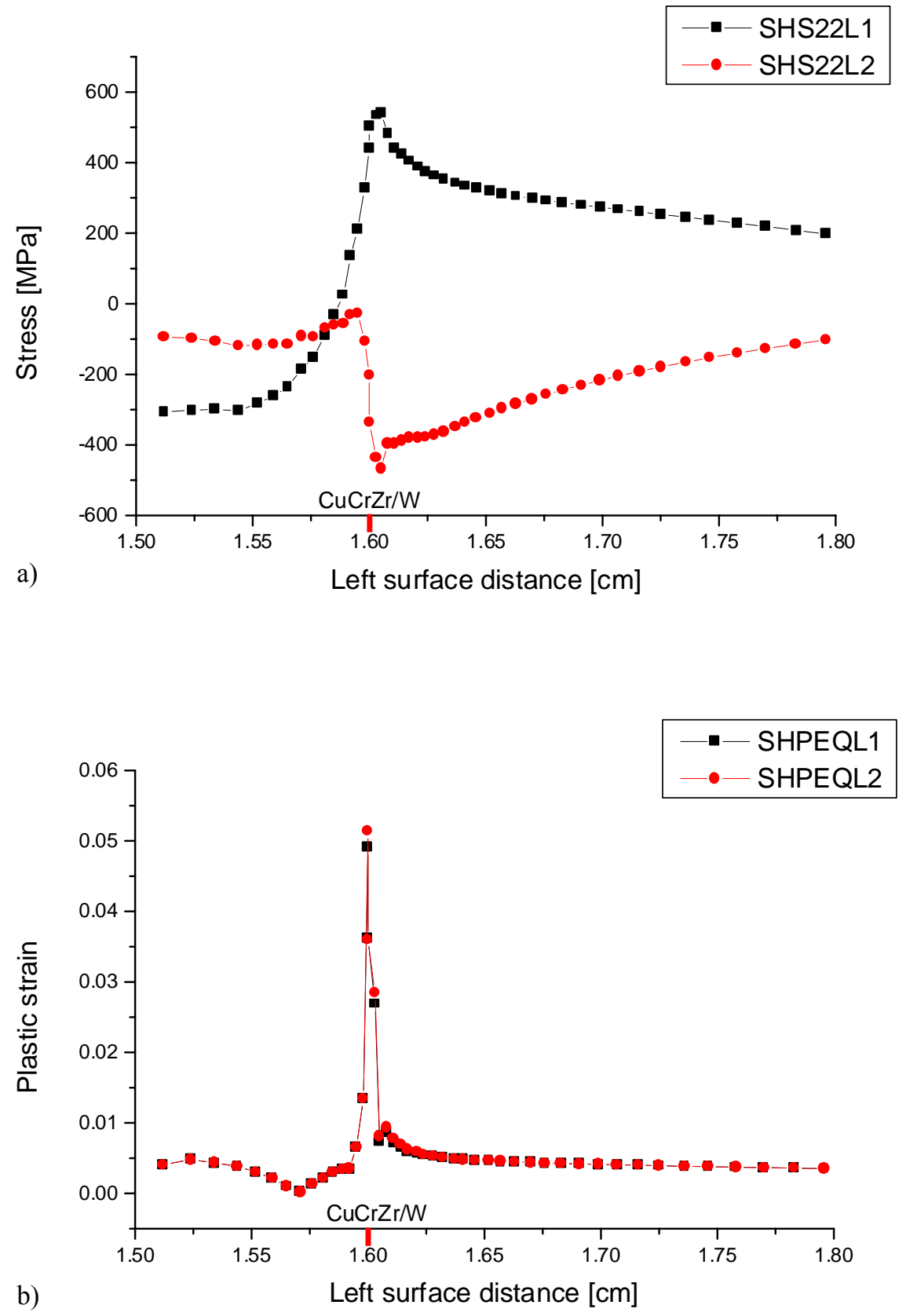

Figure 17 - a) S22 stress. b) Equivalent plastic strain. Sharp interface arhitecture along left free edge. Plots show the 2 load steps. 

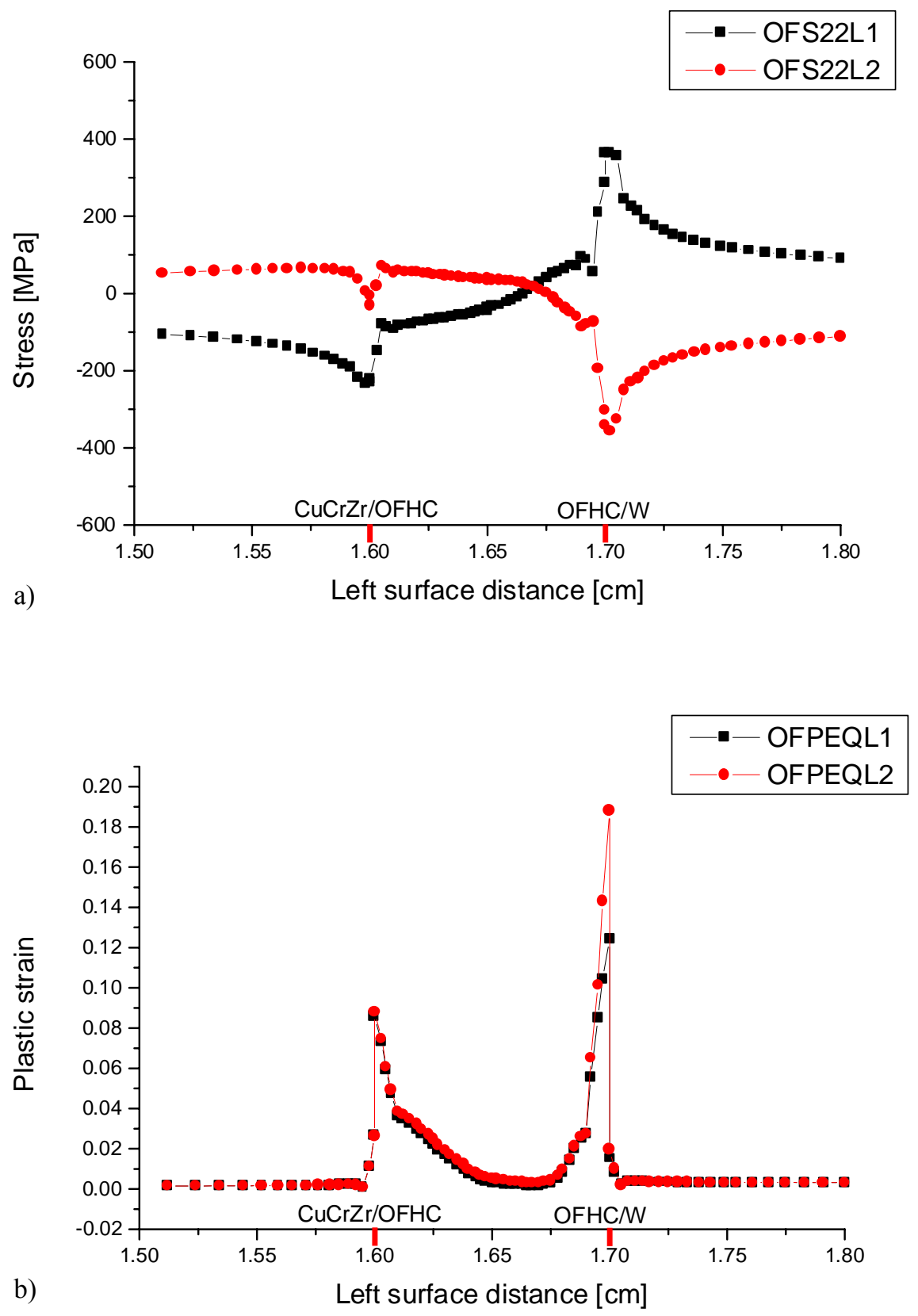

Figure 18 - a) S22 stress. b) Equivalent plastic strain. OFHC Cu interface arhitecture along left free edge. Plots show the 2 load steps. 

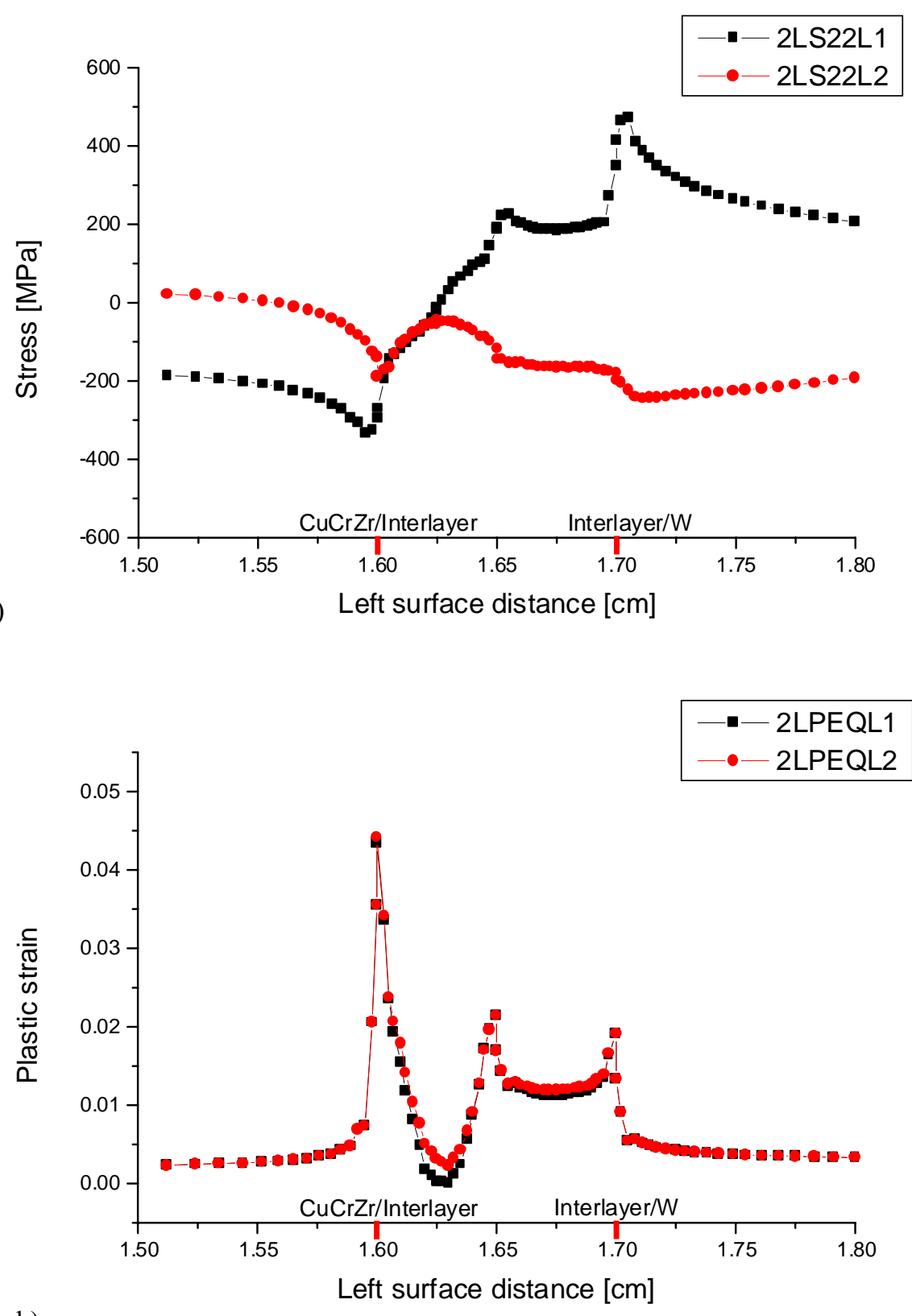

b)

Figure 19 - a) S22 stress. b) Equivalent plastic strain. $1 \mathrm{~mm} 2$ layered linear architecture along left free edge. Plots show the 2 load steps. 

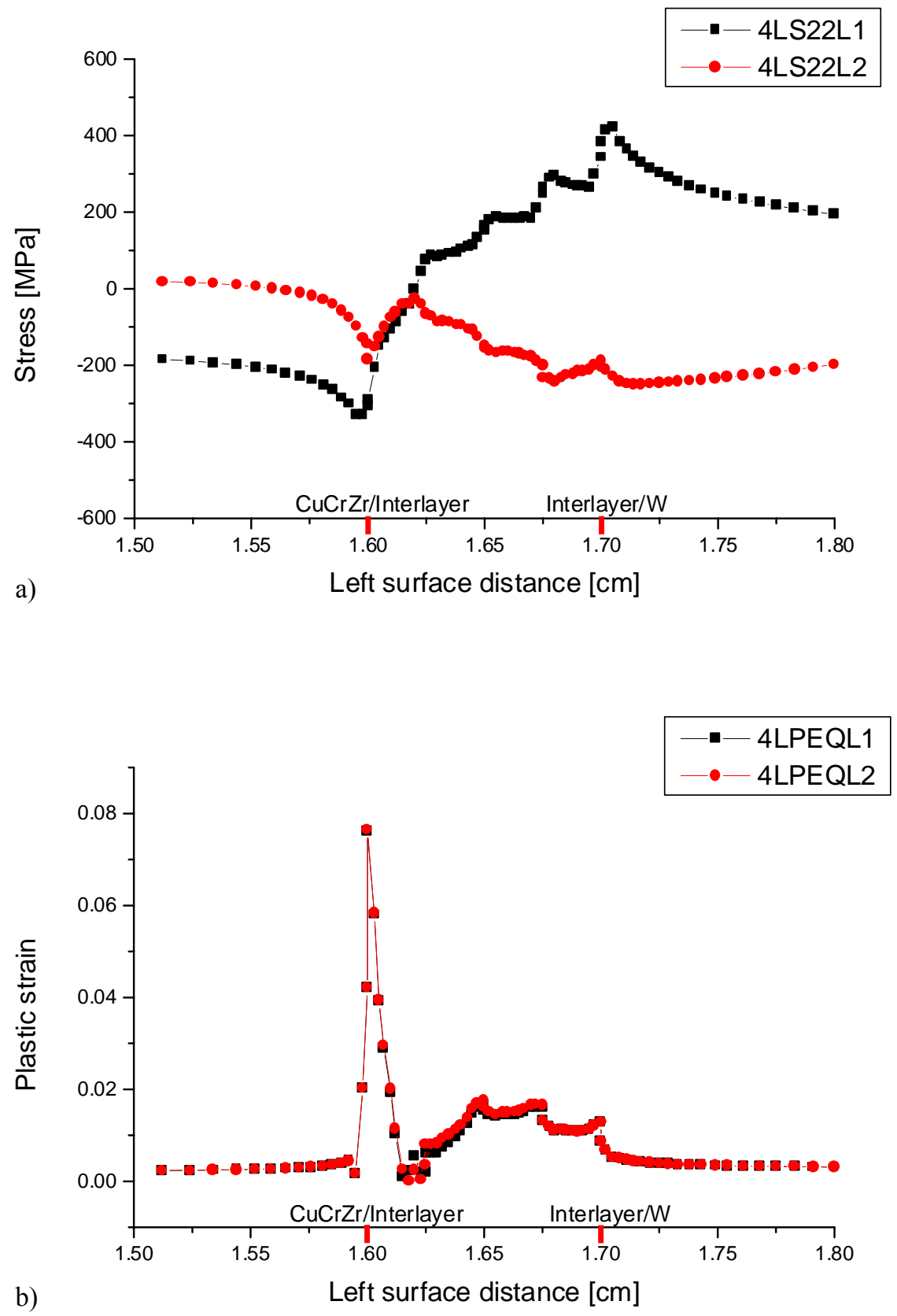

Figure 20 - a) S22 stress. b) Equivalent plastic strain. $1 \mathrm{~mm} 4$ layered linear architecture along left free edge. Plots show the 2 load steps. 


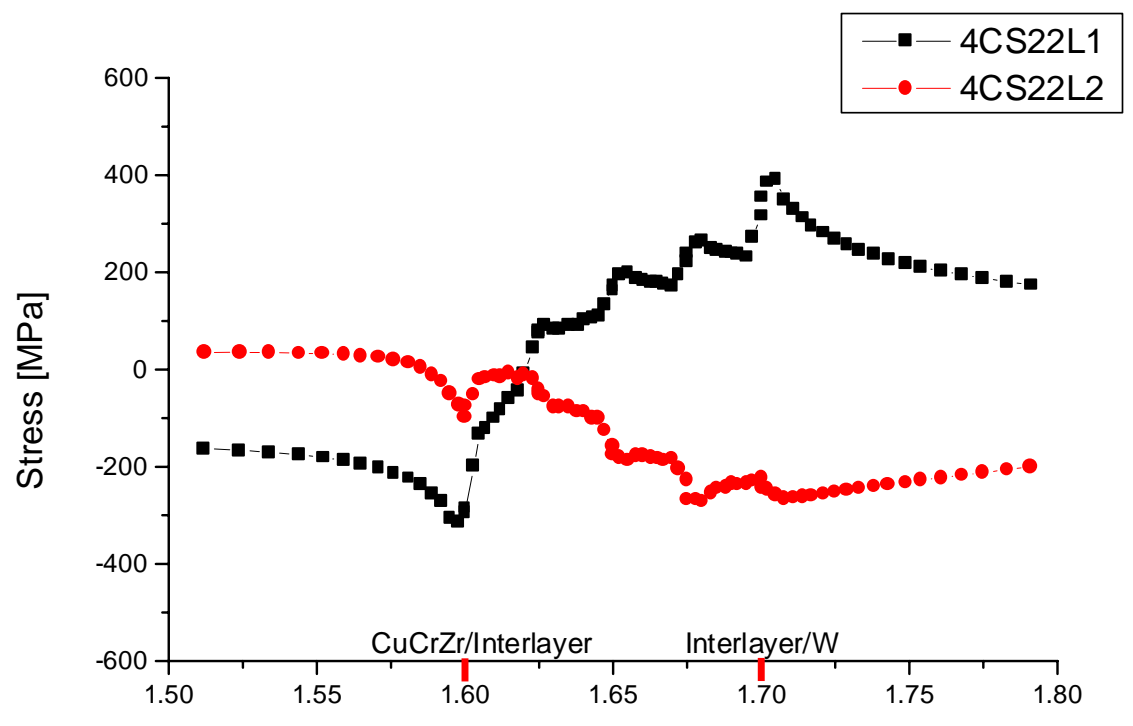

a)

Left surface distance [cm]

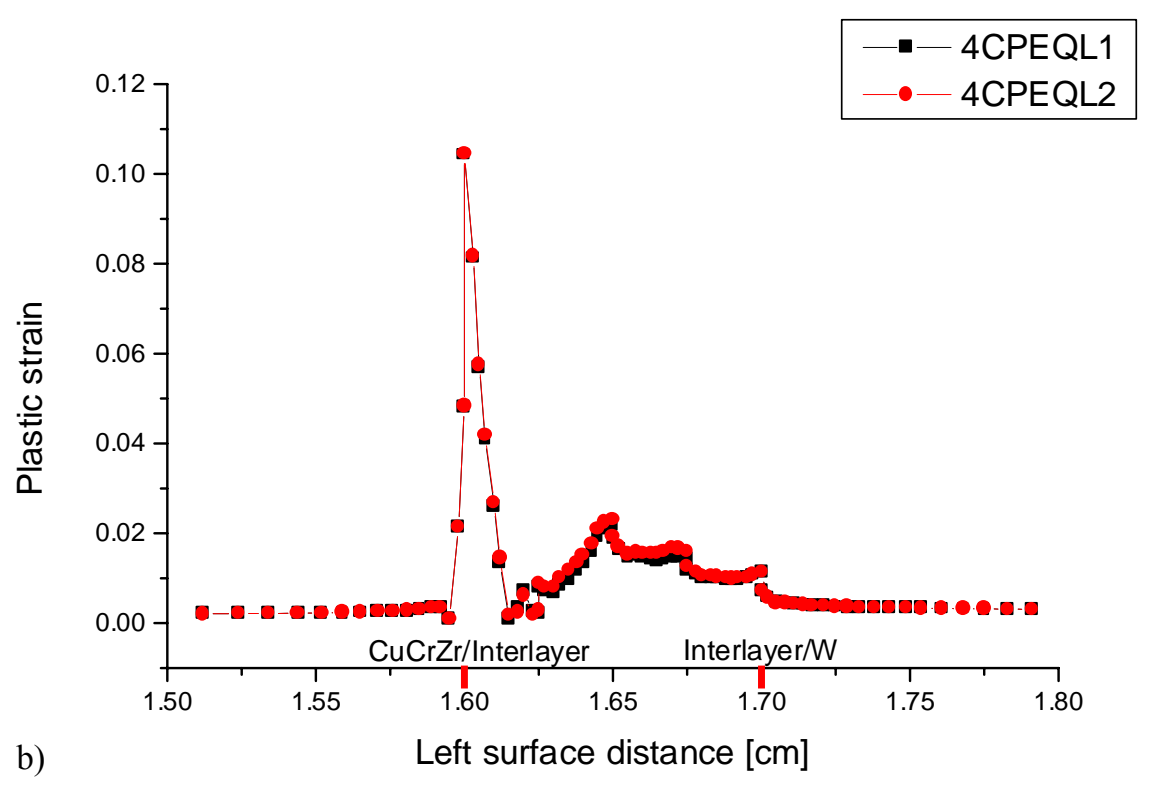

Figure 21 - a) S22 stress. b) Equivalent plastic strain. $1 \mathrm{~mm} 4$ layered $\mathrm{Cu}$ rich architecture along left free edge. Plots show the 2 load steps. 

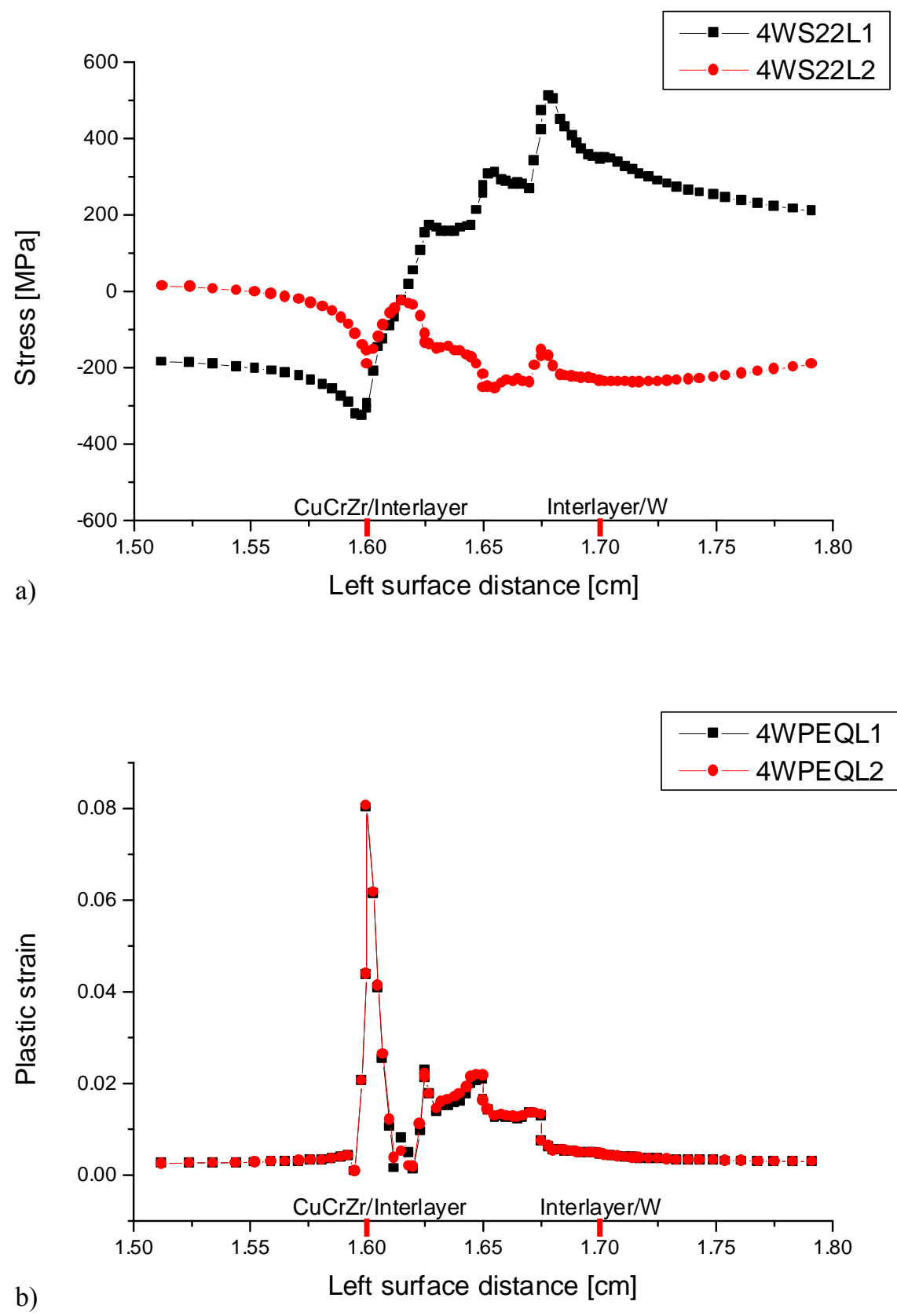

Figure 22 - a) S22 stress. b) Equivalent plastic strain. $1 \mathrm{~mm} 4$ layered W rich architecture along left free edge. Plots show the 2 load steps. 


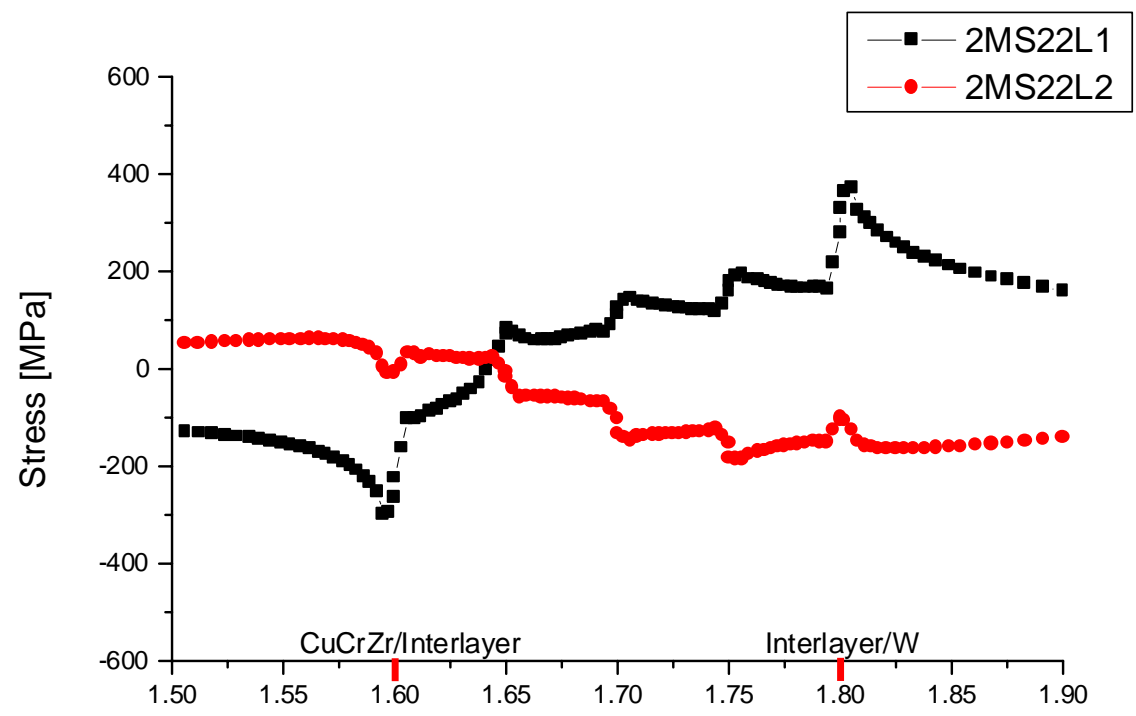

a)

Left surface distance [cm]

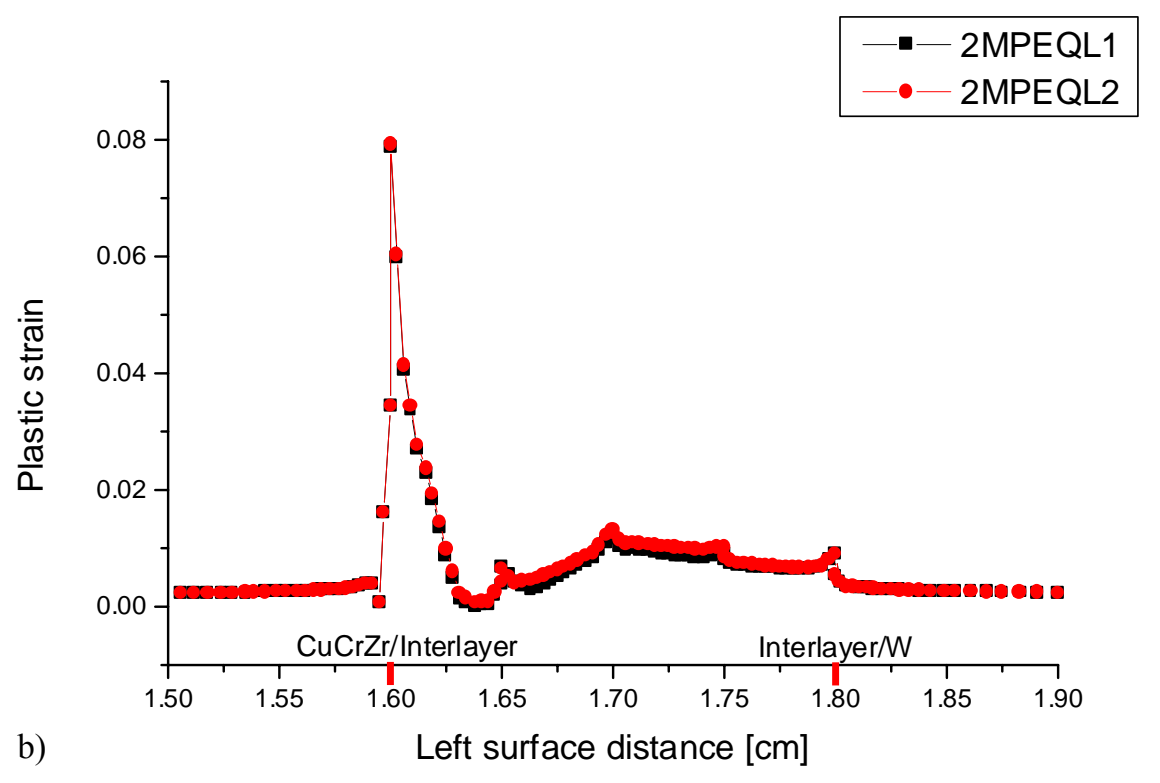

Figure 23 - a) S22 stress. b) Equivalent plastic strain. $2 \mathrm{~mm} 4$ layered $\mathrm{Cu}$ rich architecture along left free edge. Plots show the 2 load steps. 


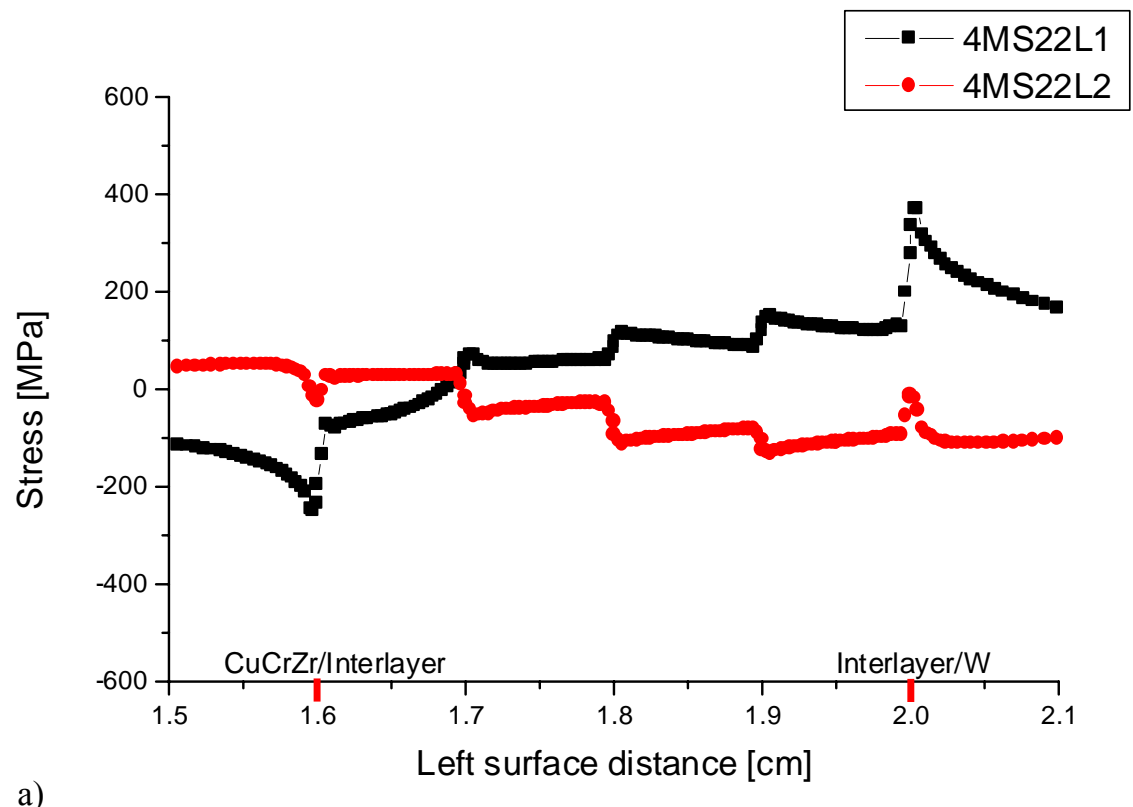

a)

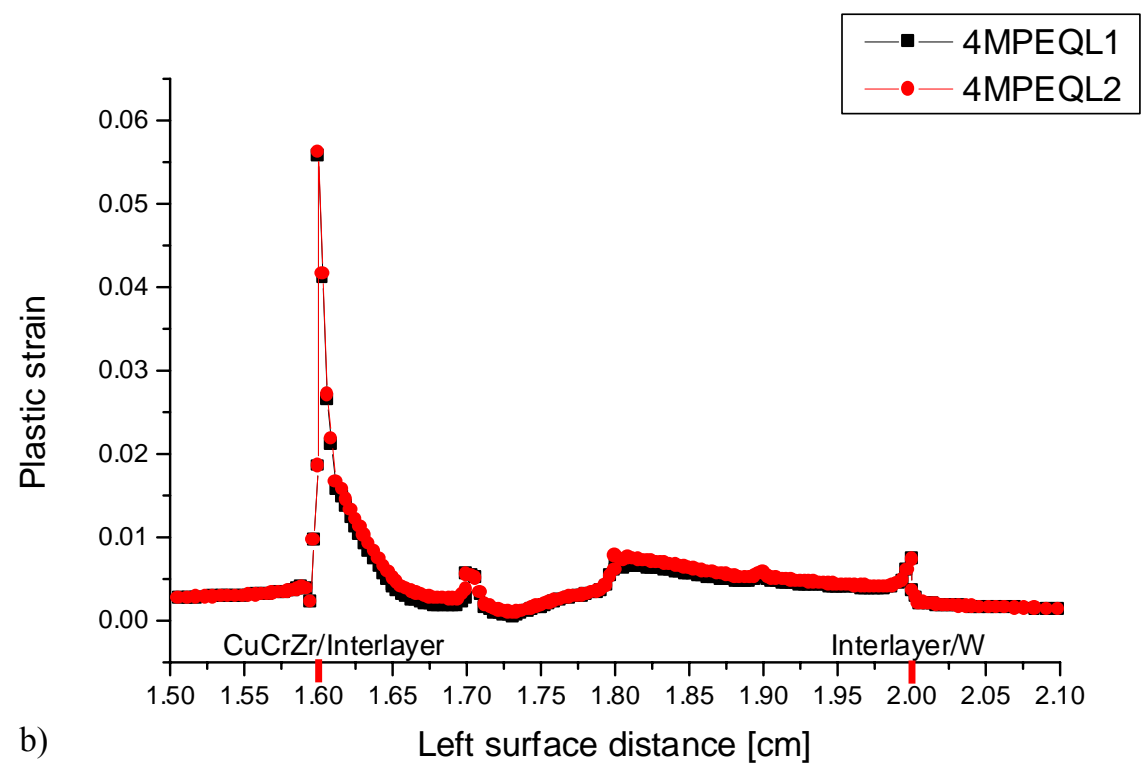

Figure 24 - a) S22 stress. b) Equivalent plastic strain. $4 \mathrm{~mm} 4$ layered $\mathrm{Cu}$ rich architecture along left free edge. Plots show the 2 load steps. 


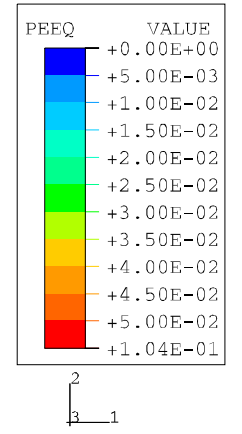

a)

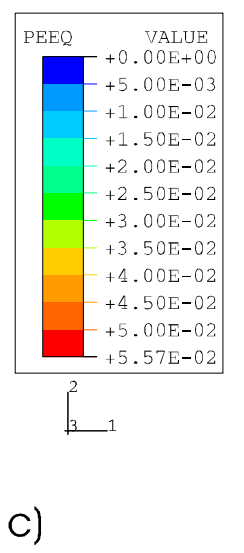

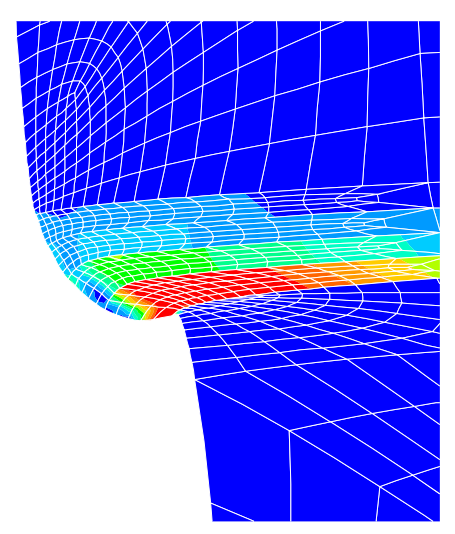

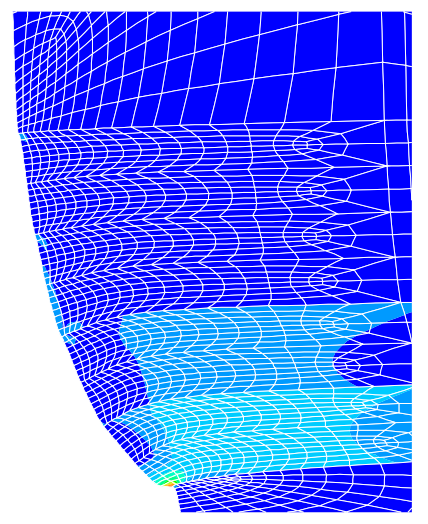

Figure 25 - Equivalent plastic strain after cooling down. a) $1 \mathrm{~mm} \mathrm{Cu}$ rich, b) $2 \mathrm{~mm} \mathrm{Cu}$ rich, c) 4 $\mathrm{mm} \mathrm{Cu}$ rich.

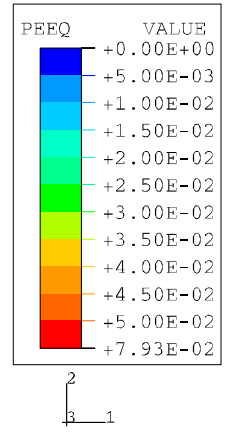

b)

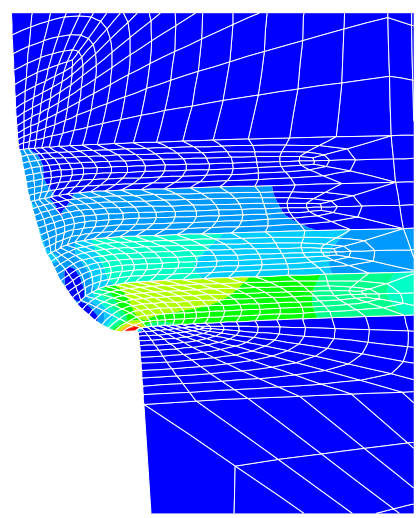


Table 5 shows a comparison of the S22 peak stresses for the different architectures, the maximum effective plastic strain is also compared. The stress reduction using $1 \mathrm{~mm} \mathrm{OFHC} \mathrm{Cu}$ interface and the $2 \mathrm{~mm} \mathrm{Cu}$ rich is very similar. But the plastic strain peak is lower for the $2 \mathrm{~mm}$ and $4 \mathrm{~mm} \mathrm{Cu}$ rich interlayers.

Table 5 - Peak S22 stress and plastic strain comparison for the different architectures.

\begin{tabular}{|l|c|c|c|c|c|c|c|c|}
\hline & SH & OF & 2L & 4L & 4C & 4W & $2 \mathrm{~mm}$ & $4 \mathrm{~mm}$ \\
\hline Peak S22 Stress (MPa) & 542 & 365 & 473 & 422 & 390 & 509 & 372 & 372 \\
\hline \% Peak Plastic Strain & & $18.8 \%$ & $4.3 \%$ & $7.6 \%$ & $10.4 \%$ & $8.0 \%$ & $7.9 \%$ & $5.6 \%$ \\
\hline \% Reduction in S22 Stress & & $33 \%$ & $13 \%$ & $22 \%$ & $28 \%$ & $6 \%$ & $31 \%$ & $31 \%$ \\
\hline
\end{tabular}

Figure 26 shows a comparison of plastic strain at the interface along the left surface for the $1 \mathrm{~mm}$ OFHC $\mathrm{Cu}$ and $1 \mathrm{~mm} \mathrm{Cu}$ rich architectures, the high plastic strain localized next to the W interface observed on the OFHC Cu interface is drastically reduced by using a graded material.

A brief model was constructed to examine the effect of the cooling channel. Figure 27 presents the stresses after cooling down from $550{ }^{\circ} \mathrm{C}$ to $25^{\circ} \mathrm{C}$ along the left surface for both geometries, with and without channel. As can be seen in Figure 27, the presence of the channel perturbs the stress fields, but does not significantly affect the magnitudes or shape of the stress distribution.

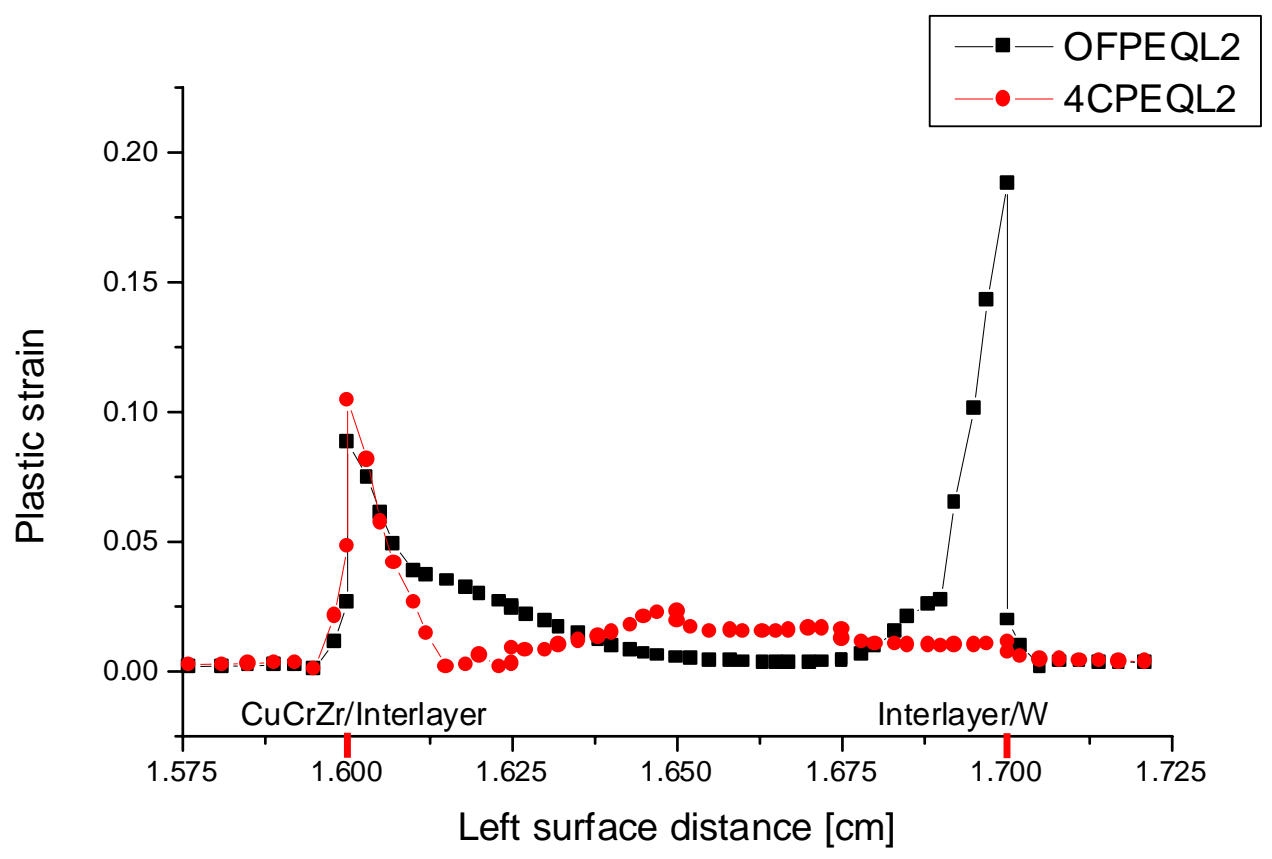

Figure 26 - Comparison of equivalent plastic strain for the $1 \mathrm{~mm} \mathrm{OFHC} \mathrm{Cu}$ and 4 layered $\mathrm{Cu}$ rich architectures after applying heat load of $5 \mathrm{MW} / \mathrm{m}^{2}$. 


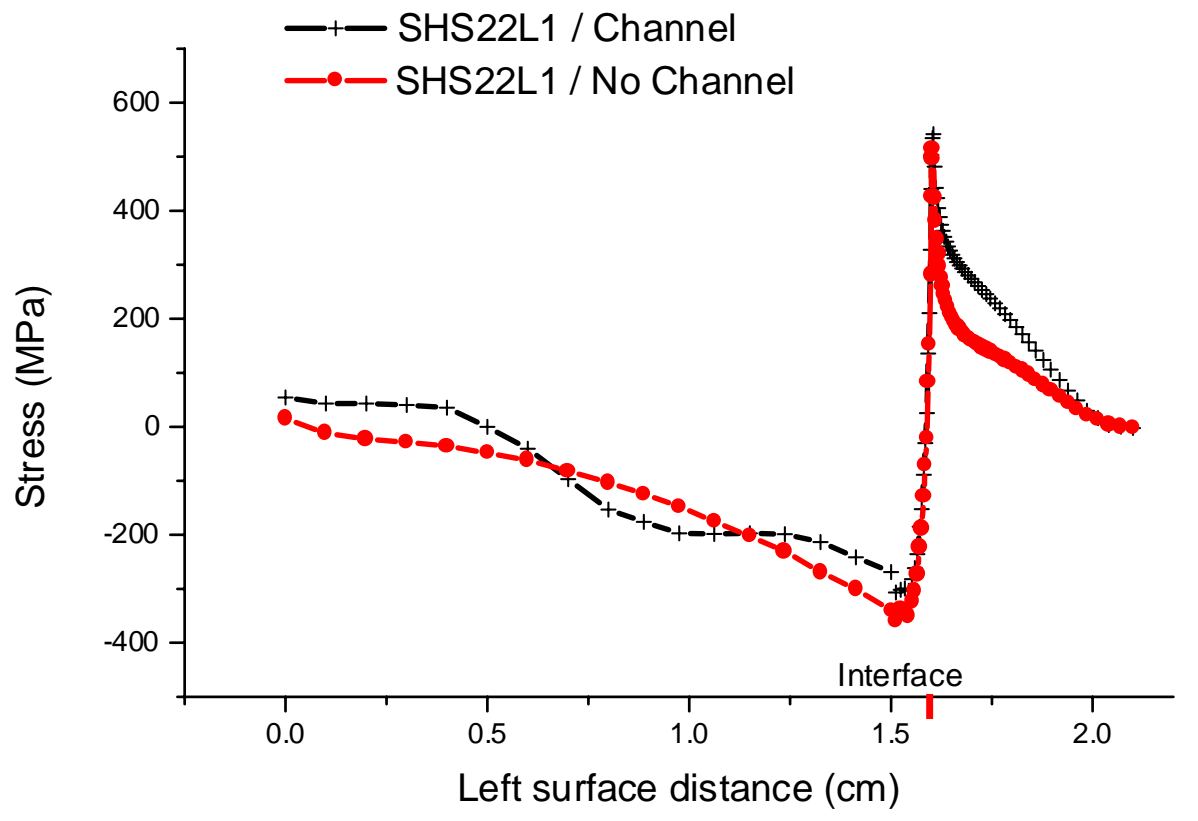

Figure 27 - S22 stress comparisson for sharp interface architecture with and without channel after cooling down.

\subsection{Discussion of Results}

The S11 stress distribution after cooling down is tensile in the CuCrZr and compressive in the $\mathrm{W}$ next to the interface due to the larger CTE of the heat sink material. The bending effect produces tensile stress at the top of the $\mathrm{W}$ armor. The maximum S11 stress at the top of the $\mathrm{W}$ occurs with the sharp interface, with a value of $271 \mathrm{MPa}$. The maximum reduction in S11 after cooling down occurs with the OFHC architecture with a value of $108 \mathrm{MPa}$. The $1 \mathrm{~mm} \mathrm{Cu}$ rich shows a S11 value of $173 \mathrm{MPa}$ at the top of the $\mathrm{W}$.

Using any of the different materials analyzed as an interlayer (Figure 5) to make the $\mathrm{W} / \mathrm{CuCrZr}$ joint results in bending reduction comparing to the sharp interface. This decrease in bending reduces the S11 stress at the $\mathrm{W}$ armor top. The bending reduction can be observed in the different contour plots (Figure 13 and appendix A). The stress component may produce failure in the form of a crack propagating perpendicular to the interface and with origin at the top of the hard material after cooling down. The stress S22 at the singular corner is larger than S11 at the armor top for all the structures. Based on this, a fracture originating at the singular corner is more likely than anywhere else in the structure, thus S22 stresses around this area must be analyzed in more detail.

The S11 stresses obtained when the temperature is reduced from $550{ }^{\circ} \mathrm{C}$ to $25{ }^{\circ} \mathrm{C}$ are similar to the pattern of stresses predicted by the analytical theory [18,19]. Tensile and compressive S11 stresses are found next to the joint at the higher and lower CTE materials respectively and change in sign of the S11 stresses due to bending is observed. 
Because no external loads are applied at the surfaces in this model the stress perpendicular to any surface must be equal to zero. This condition is not achieved at the singular corner area, but a trend to zero in S11 is observed as we approach this region in almost all the geometries, with exception of the soft OFHC Cu interlayer and the $2 \mathrm{~mm}$ and $4 \mathrm{~mm}$ interlayer thick architectures. Therefore, some error may be present in the stress and strain results around the singular corner. Nevertheless, the same element size is used around this critical area on the different meshes and the results obtained are used on a comparative basis to find the architecture which gives the maximum stress reduction.

A reversal in stresses is observed after applying the heat load. Now tensile S11 stress is observed in the $\mathrm{W}$ next to the interface and compressive stress in the $\mathrm{CuCrZr}$ or interlayer. S22 also changes from tensile to compressive around the singular corner.

The stress reversal observed may be explained as follows. Applying the heat load at the armor top and removing heat by water flowing through the cooling channel results in a temperature gradient, in which the high temperature at the top decreases gradually towards the channel. In presence of this temperature distribution, bending would be expected for the tungsten if it is not constrained by the material to which is joined. The larger thermal expansion coefficient of the $\mathrm{CuCrZr}$ or the material joined to the armor on the other architectures, induces tensile S11 stress in the tungsten next to the joint and also limits the bending on the armor generating a clamping effect around the singular corner with compressive S22 stress.

After cooling down, the maximum stress reduction is observed using the OFHC $1 \mathrm{~mm}$ interlayer. The larger plastic strain is observed using the OFHC. Applying the heat load results in further increase of the plastic strain.

Larger S22 stresses than those observed using the OFHC are present using the different graded interlayer architectures, but the strain peak is decreased. The larger strain distribution, observed in the copper rich graded interlayer, does not change after applying the heat load. This could indicate an advantage of using the graded interlayer versus the OFHC, since the increase in plastic strain after cooling down may suggest the possibility of failure under cyclic load. The plastic strain increases from $12.4 \%$ to $18.8 \%$ by applying the heat load when using the $1 \mathrm{~mm}$ $\mathrm{OFHC} \mathrm{Cu}$ as a interlayer.

A redistribution of the plastic strain is observed removing the plastic strain peak from the area next to the $\mathrm{W}$ interface by using a graded interlayer and the magnitude of this peak is decreased. Increasing the thickness of the $\mathrm{Cu}$ rich graded interlayer from $1 \mathrm{~mm}$ to $2 \mathrm{~mm}$ results in $\mathrm{S} 22$ stress reduction and plastic strain reduction is also observed. Increasing the thickness to $4 \mathrm{~mm}$ has no effect on the tensile S22 stress in the singular corner but the plastic strain is further decreased. No increase in plastic strain is observed for these architectures after the heat load of $5 \mathrm{MW} / \mathrm{m}^{2}$ is applied, contrary to what is observed for the OFHC $\mathrm{Cu}$ interlayer. This could be an advantage to using the graded architecture.

A difference in the S22 stress distribution was obtained for the analysis without channel after cooling down. However, the stresses were not significantly altered. In particular, the magnitude of the critical S22 stress at the singular corner was not affected. 


\subsection{MECHANICAL AND PHYSICAL PROPERTIES OF VPS DEPOSITS}

During this stage of the investigation, vacuum plasma spray forming techniques were used to produce both copper and tungsten deposits for physical and mechanical properties testing.

\subsection{VPS Copper Evaluation}

To evaluate the effect oxygen content had on the mechanical and physical properties of VPS copper deposits, two copper feedstock powders were evaluated. Both powders were a -325 mesh particle size, and both were originally a high conductivity, oxygen free grade packaged in inert atmosphere containers. However, one set of powders was opened and exposed to atmosphere for a minimum of one week prior to vacuum plasma spraying. The other set of powder was not opened until just prior to loading in the powder feeder for spraying. Visual inspection of the two powders showed the unexposed powder had a more pinkish tint to the powder as compared to the exposed powder, which had a darker pink tint. Oxygen content of the two powders (Lo and Hi oxygen contents) are listed in Table 6 . Note the Hi oxy powder was the one exposed to atmosphere a minimum of one week prior to use.

Table 6 - Copper feedstock powders used during this investigation.

\begin{tabular}{|c|c|c|}
\hline ID & Description & Oxygen Content (ppm) \\
\hline Lo oxy & Low oxygen copper powder, -325 mesh & 928 \\
\hline Hi oxy & High oxygen copper powder, -325 mesh & 1896 \\
\hline
\end{tabular}

During vacuum plasma spray forming of the copper deposits, $75 \mathrm{~mm}$ x $150 \mathrm{~mm}$ steel substrates were used. A typical set of parameters used to produce the deposits is listed in Table 7. Prior to spraying, the chamber was evacuated a minimum of twice and backfilled with high purity argon. The plasma gun was then started and used to preheat the substrate. To determine the effect of substrate temperature on the properties of the deposits, two different preheat temperatures were evaluated. A thermocouple was attached to each substrate to accurately measure the preheat temperature. After obtaining the desired preheat temperature, powder was fed to the plasma nozzle, where it was melted and accelerated toward the substrate. A rectilinear pattern was used during spraying and all movement was computer numerically controlled. The copper was typically deposited to a thickness of $\sim 6 \mathrm{~mm}$ to enable subsequent machining of samples for mechanical and physical properties testing. Once the deposit had cooled to room temperature, it was removed from the chamber.

Table 7 - Parameters for VPS copper deposition.

\begin{tabular}{|c|c|}
\hline Parameter & Value \\
\hline Primary Gas, Ar $(\mathrm{slm})$ & 123 \\
\hline Secondary Gas, $\mathrm{H}_{2}(\mathrm{slm})$ & 3.5 \\
\hline Powder Gas, $\mathrm{Ar}(\mathrm{slm})$ & 5 \\
\hline Powder Feed Rate $(\mathrm{rpm})$ & 2.50 \\
\hline Amps $(\mathrm{A})$ & 100 \\
\hline Volts $(\mathrm{V})$ & 58 \\
\hline Stand-off $(\mathrm{cm})$ & 18 \\
\hline Pressure (torr) & 35 \\
\hline Pre-heat Temperature $\left({ }^{\circ} \mathrm{C}\right)$ & 800 and 1100 \\
\hline
\end{tabular}


A photomicrograph of a VPS copper deposit in the as-sprayed condition is shown in Figure 28. Note a recrystallized structure was produced as opposed to a splat-like structure, which is typical for conventional plasma sprayed deposits.

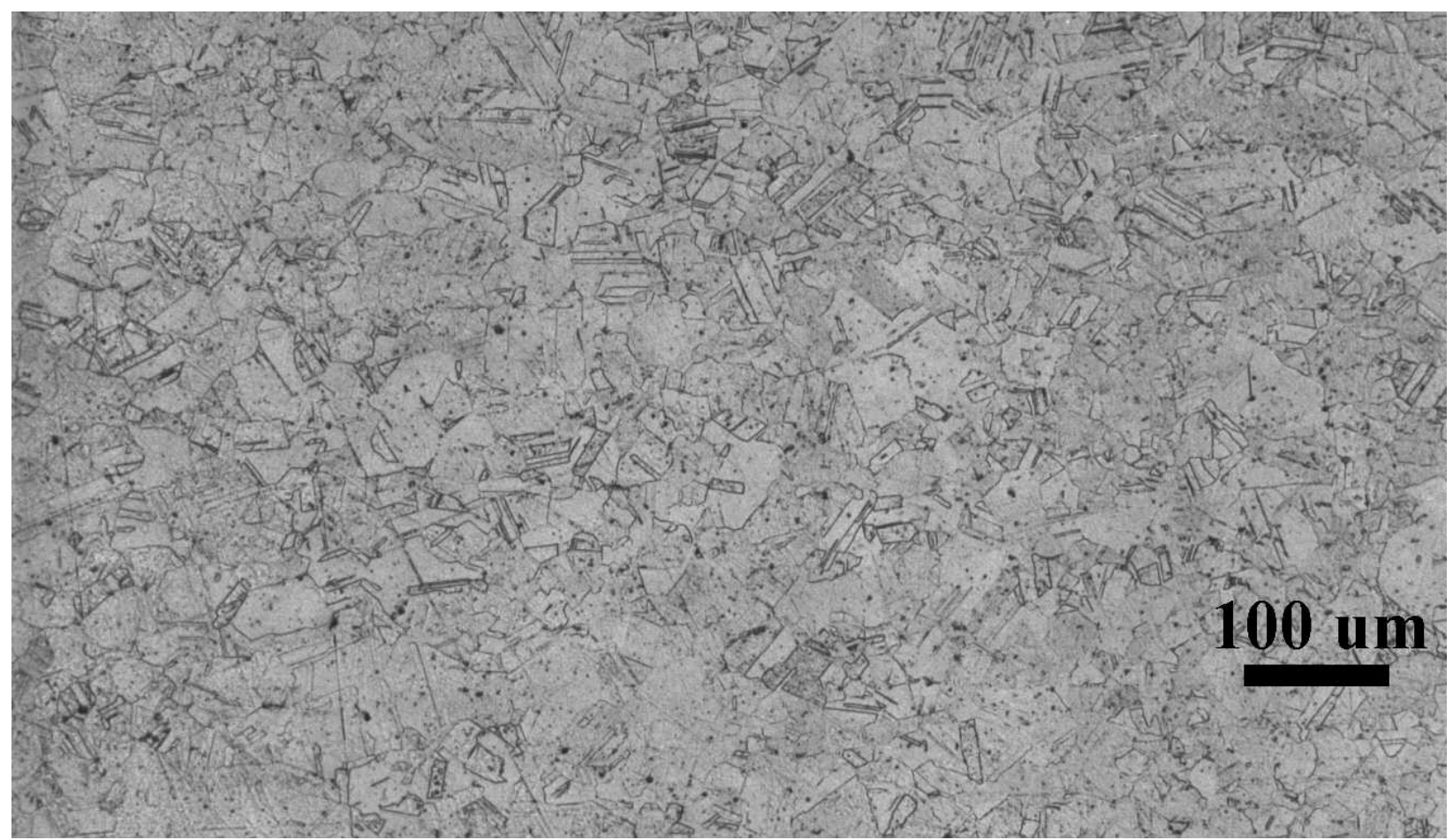

Figure 28 - Photomicrograph showing VPS copper deposit in the as-sprayed condition.

\subsubsection{Post-Spray Heat Treatment}

Three post-spray heat treatments were evaluated to determine their effects on the properties of the VPS formed copper deposits. A list of the heat treatments that were used is given in Table 8 .

Table 8 - Post-spray heat treatments evaluated for VPS copper.

\begin{tabular}{|c|c|}
\hline ID & Description \\
\hline VA & Vacuum anneal: 1) $750^{\circ} \mathrm{C}, 1 \mathrm{hr},<10^{-4}$ torr \\
& 2) $900^{\circ} \mathrm{C}, 1 \mathrm{hr},<10^{-4}$ torr \\
\hline HIP & Hot isostatic pressure: $900^{\circ} \mathrm{C}, 3 \mathrm{hr}, 20 \mathrm{ksi}$ \\
\hline VA + HIP & Two step heat treatment: 1$) \mathrm{VA}$ and 2) HIP \\
\hline
\end{tabular}

\subsubsection{Characterization}

To characterize the as-sprayed and heat treated VPS copper deposits, mechanical and physical properties were measured. Water immersion techniques were used to determine the density values of each material. Laser flash methods were used to measure thermal diffusivities from room temperature to $1000^{\circ} \mathrm{C}$. The measured densities and diffusivities along with theoretical specific heats were then used to calculate the thermal conductivity values. The mechanical properties were determined using standard, flat tensile bars. The tensile tests were conducted at both room temperature and $300^{\circ} \mathrm{C}$. 


\subsubsection{VPS Copper Mechanical Properties Results and Discussion}

The results of the mechanical properties tests are shown in Table 9. Listed in the table are the ultimate tensile strength, yield strength, and elongation for each of the four conditions, i.e., 1) As-Sprayed, 2) Vacuum Annealed, 3) Vacuum Anneal and HIP, and 4) HIP. Room temperature and elevated temperature results are listed for both the Hi and Lo oxy feedstock powders and the two different preheat temperatures. Figures $29-37$ plot the tensile results for the different conditions as a function of feedstock powder and preheat temperature to determine the effects of oxygen in the starting powder and substrate temperature on the tensile properties.

Figure 29 plots the room temperature yield strength for the different sample conditions as a function of feedstock powder and preheat temperature. This figure shows of the as-sprayed samples the Hi oxy, 800 sample had the highest yield strength (14, $200 \mathrm{psi})$, while the three heat treated samples had yield strengths that varied from 10,300-11,000 psi. Vacuum annealing resulted in an increase in the room temperature yield strength for both of the Hi oxy samples. In contrast, vacuum annealing resulted in little change of the yield strength for both of the Lo oxy samples. For the vacuum anneal/HIP and HIP samples, the Hi oxy, 800 samples saw a significant reduction in yield strength, while the Hi oxy, 1100 sample saw a significant increase as compared to the as-sprayed condition. For comparison, the Lo oxy samples experienced a slight decrease in yield strength from the vacuum anneal/HIP treatment and little change in the yield strength for the samples in the HIP condition as compared to the as-sprayed yield strength. In general, the Lo oxy feedstock powder produced samples with more consistent yield strength values independent of the sample condition and preheat temperature. In contrast, the Hi oxy feedstock material resulted in higher yield strength values with significant variation depending on heat treatment condition and preheat temperature.

Figure 30 plots the room temperature ultimate tensile strength for the different sample conditions as a function of feedstock powder and preheat temperature. This figure shows both feedstock powder and preheat temperature had little effect on the room temperature ultimate tensile strength of VPS copper. Similarly, the post-spray heat treatments had little effect on the room temperature tensile strength.

Figure 31 plots the elevated temperature ultimate tensile strength for the different sample conditions as a function of feedstock powder and preheat temperature. The figure shows the elevated ultimate tensile strength for the Lo oxy feedstock powders were consistently around $20,000 \mathrm{psi}$ independent of preheat temperature or sample condition. In contrast, the Hi oxy feedstock powder showed a significant increase in elevated temperature ultimate tensile strength as a result of post-spray heat treating.

Figure 32 plots both the room temperature and elevated temperature ultimate tensile strengths for the Lo oxy feedstock powder. Again, this figure shows there is little dependence of the tensile strength on preheat temperature or sample condition for the Lo oxy feedstock powder. Figure 33 plots both the room temperature and elevated temperature ultimate tensile strengths for the Hi oxy feedstock powder. Unlike the Lo oxy feedstock powder, the Hi oxy feedstock powder does show some dependency of the ultimate tensile strength on post-spray heat treatment condition as compared to the as-sprayed condition, particularly for the elevated temperature ultimate tensile strength. 
Table 9 - Tensile test results for vacuum plasma sprayed copper deposits.

\begin{tabular}{|c|c|c|c|c|c|c|c|c|c|}
\hline \multirow{2}{*}{ Condition } & \multirow{2}{*}{ Property } & \multicolumn{4}{|c|}{ Room Temperature } & \multicolumn{4}{|c|}{ Elevated Temperature $\left(300^{\circ} \mathrm{C}\right)$} \\
\hline & & Hi oxy, 800 & Hi oxy, 1100 & Lo oxy, 800 & Lo oxy, 1100 & Hi oxy, 800 & Hi oxy, 1100 & Lo oxy, 800 & Lo oxy, 1100 \\
\hline \multirow{3}{*}{ 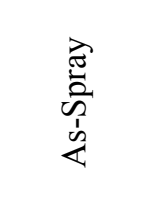 } & Tensile Strength (psi) & 30,133 & 30,800 & 30,025 & 30,767 & 17,167 & 15,700 & 20,150 & 19,500 \\
\hline & Yield Strength (psi) & 14,200 & 10,300 & 10,575 & 11,000 & - & - & - & - \\
\hline & Elongation (\%) & 40 & 43 & 51 & 49 & 17 & 18 & 45 & 43 \\
\hline \multirow{3}{*}{ 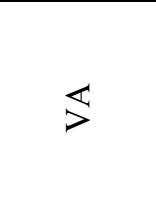 } & Tensile Strength (psi) & 32,367 & 32,500 & 31,150 & 32,267 & 20,833 & 18,367 & 21,400 & 19,233 \\
\hline & Yield Strength (psi) & 15,000 & 13,478 & 10,550 & 10,800 & - & - & - & - \\
\hline & Elongation (\%) & 45 & 43 & 52 & 50 & 33 & 27 & 47 & 53 \\
\hline \multirow{3}{*}{ 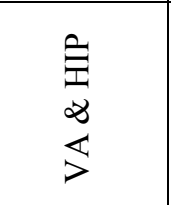 } & Tensile Strength (psi) & 30,700 & 33,633 & 30,025 & 29,367 & 19,633 & 24,067 & 20,450 & 19,133 \\
\hline & Yield Strength (psi) & 11,500 & 14,467 & 8,815 & 9,042 & - & - & - & - \\
\hline & Elongation (\%) & 48 & 49 & 54 & 55 & 31 & 44 & 51 & 53 \\
\hline \multirow{3}{*}{ 主 } & Tensile Strength (psi) & 30,967 & 33,867 & 31,575 & 31,833 & 18,500 & 21,300 & 19,500 & 20,000 \\
\hline & Yield Strength (psi) & 11,300 & 14,700 & 10,075 & 10,967 & - & - & - & - \\
\hline & Elongation (\%) & 52 & 53 & 56 & 56 & 24 & 37 & 54 & 55 \\
\hline
\end{tabular}




\section{VPS Copper Yield Strength (RT)}

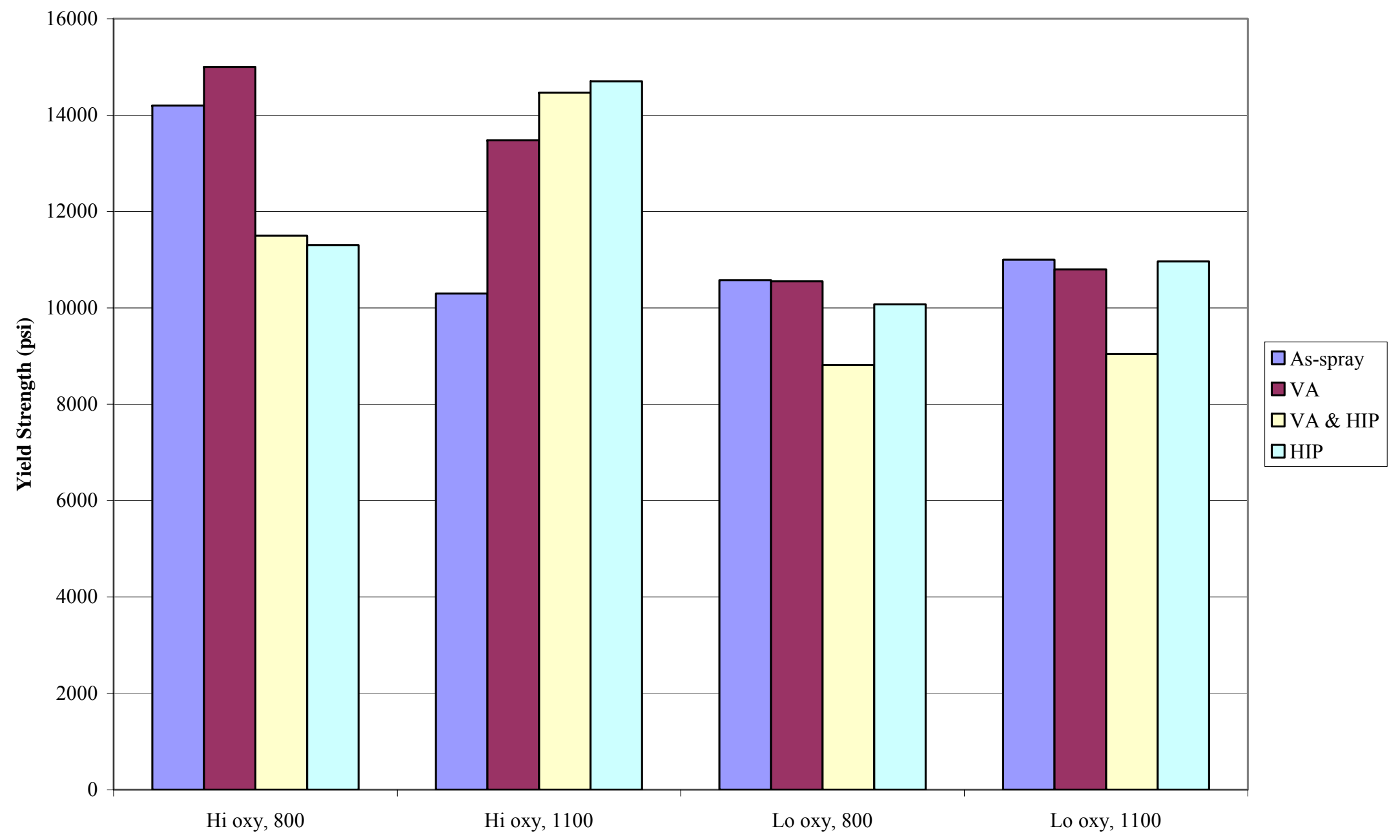

Figure 29 - Plot showing room temperature yield strength of VPS copper in different conditions as a function of feedstock powder and preheat temperature. 


\section{VPS Copper Tensile Strength (RT)}

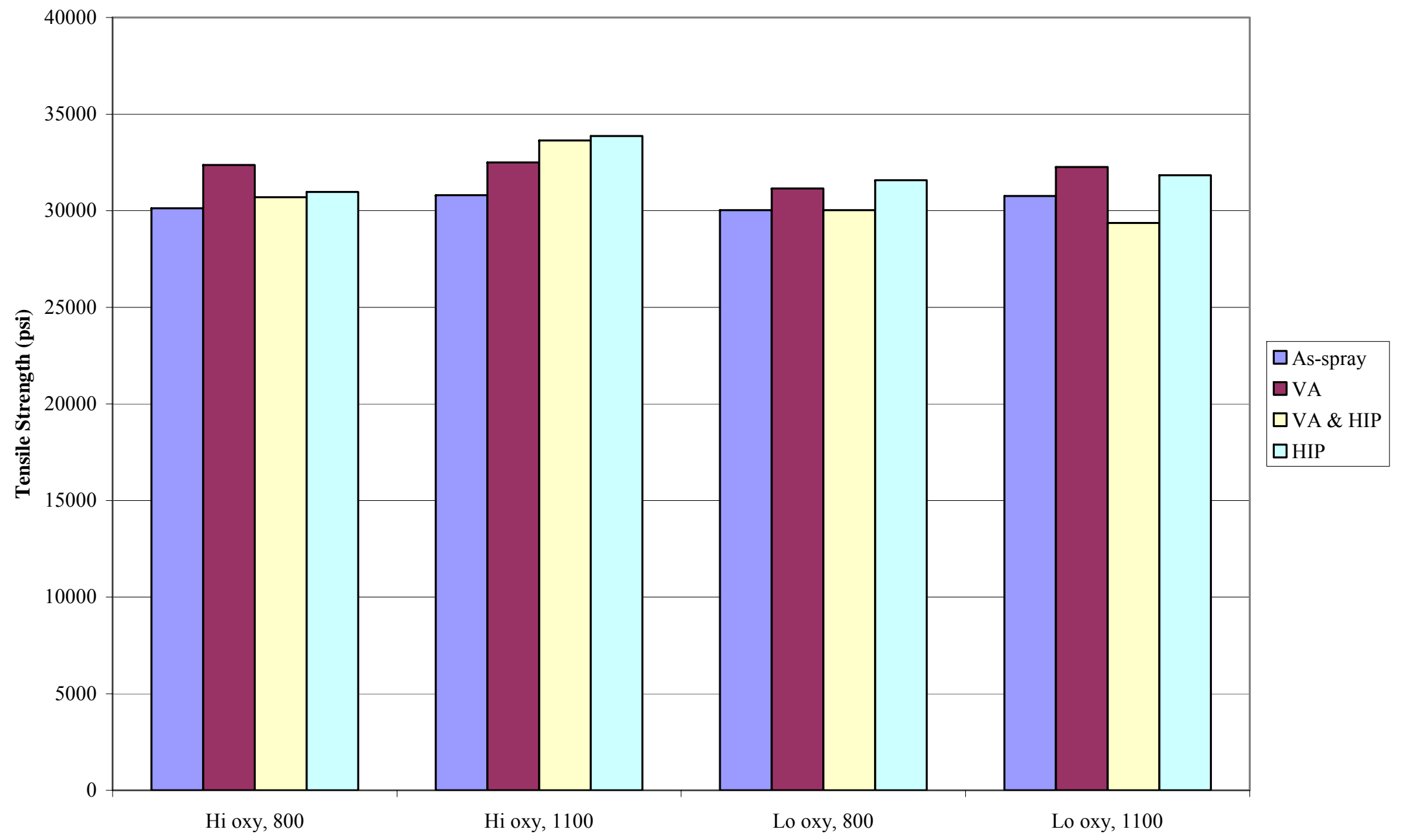

Figure 30 - Plot showing room temperature ultimate tensile strength of VPS copper in different conditions as a function of feedstock powder and preheat temperature. 


\section{VPS Copper Tensile Strength (300C)}

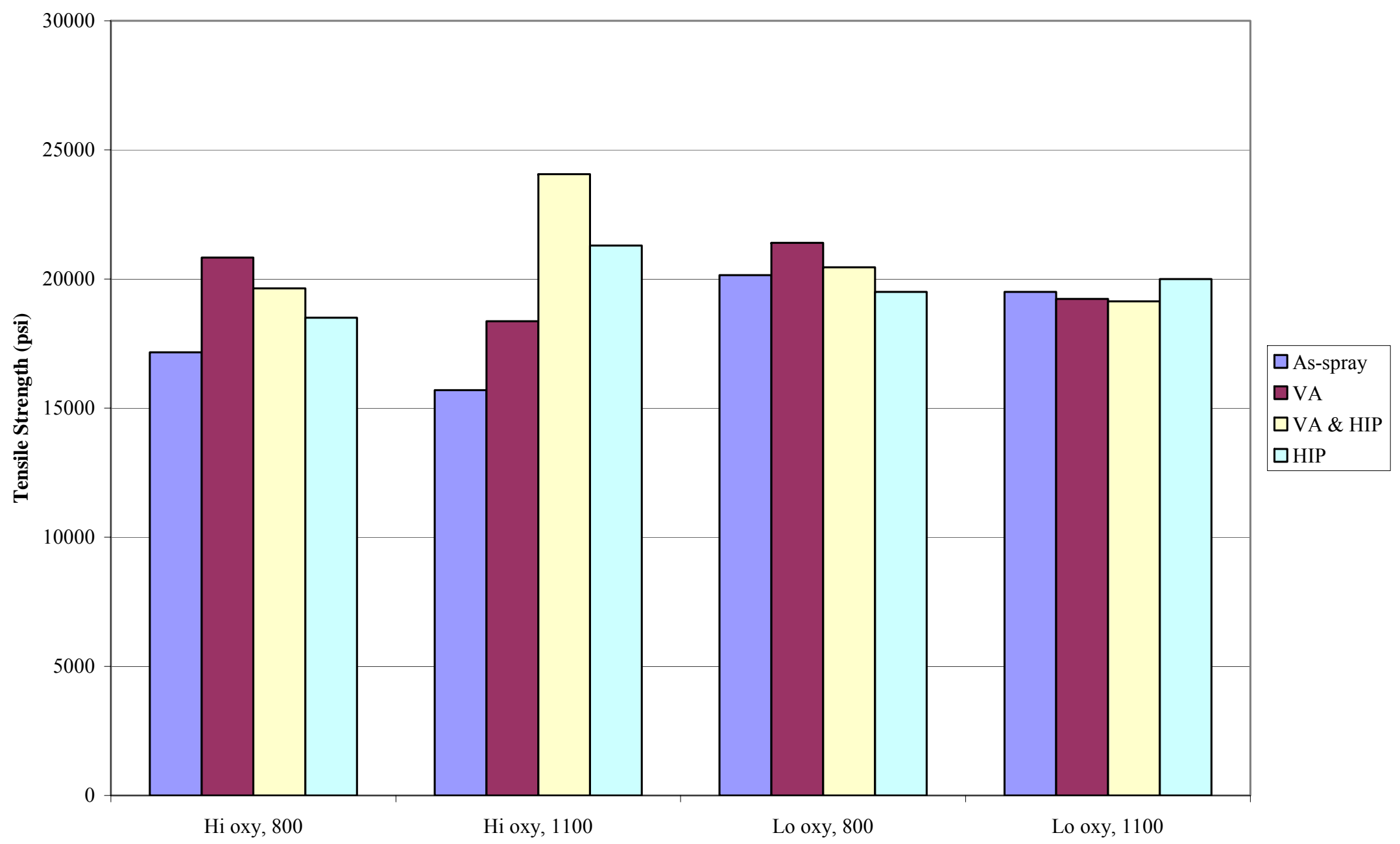

Figure 31 - Plot showing elevated temperature ultimate tensile strength of VPS copper in different conditions as a function of feedstock powder and preheat temperature. 


\section{VPS Cu Tensile Strength (Lo oxy)}

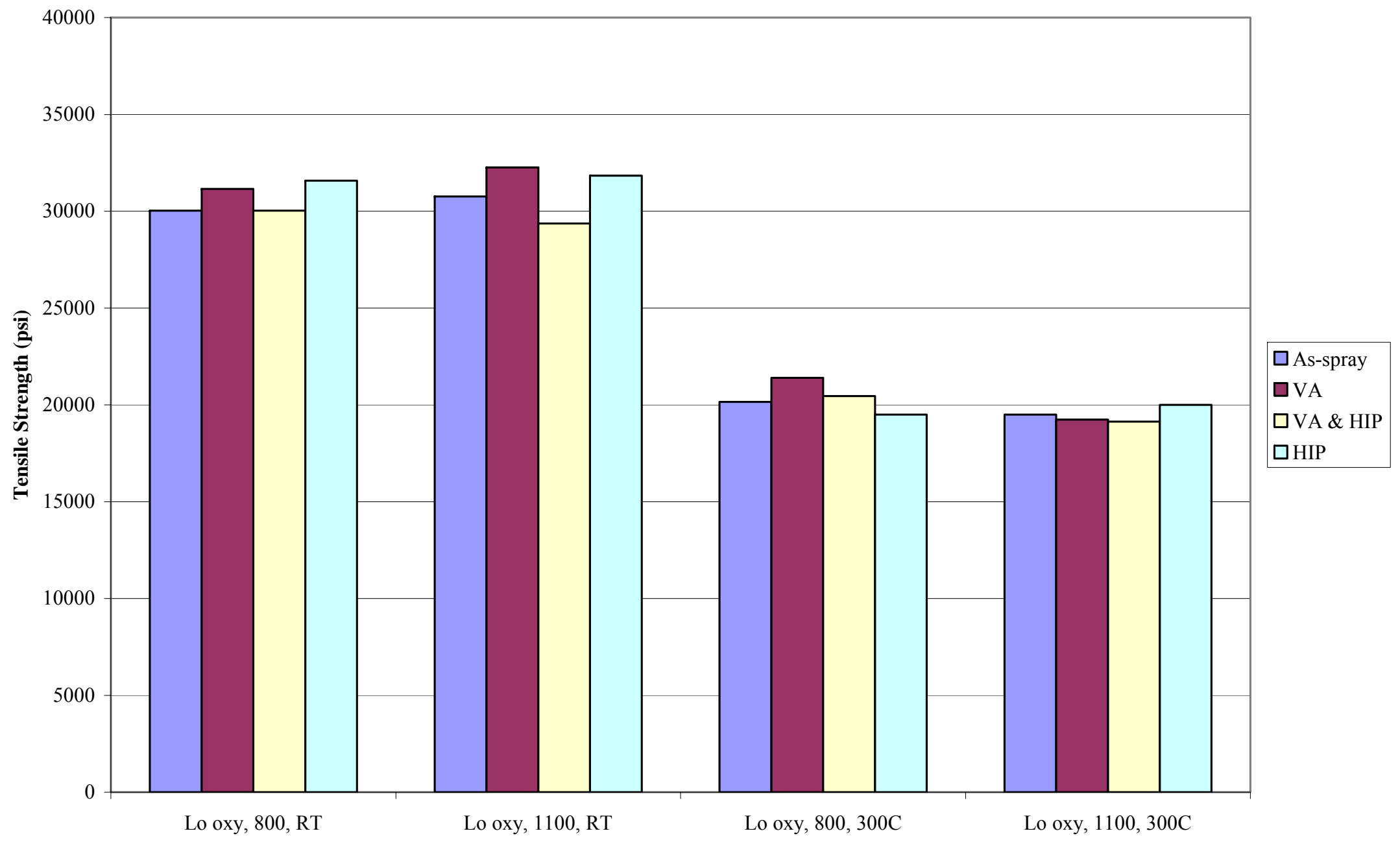

Figure 32 - Plot showing room temperature and elevated temperature ultimate tensile strength of VPS copper in different conditions produced with Lo oxy feedstock powder as a function of preheat temperature. 


\section{VPS Copper Tensile Strength (Hi oxy)}

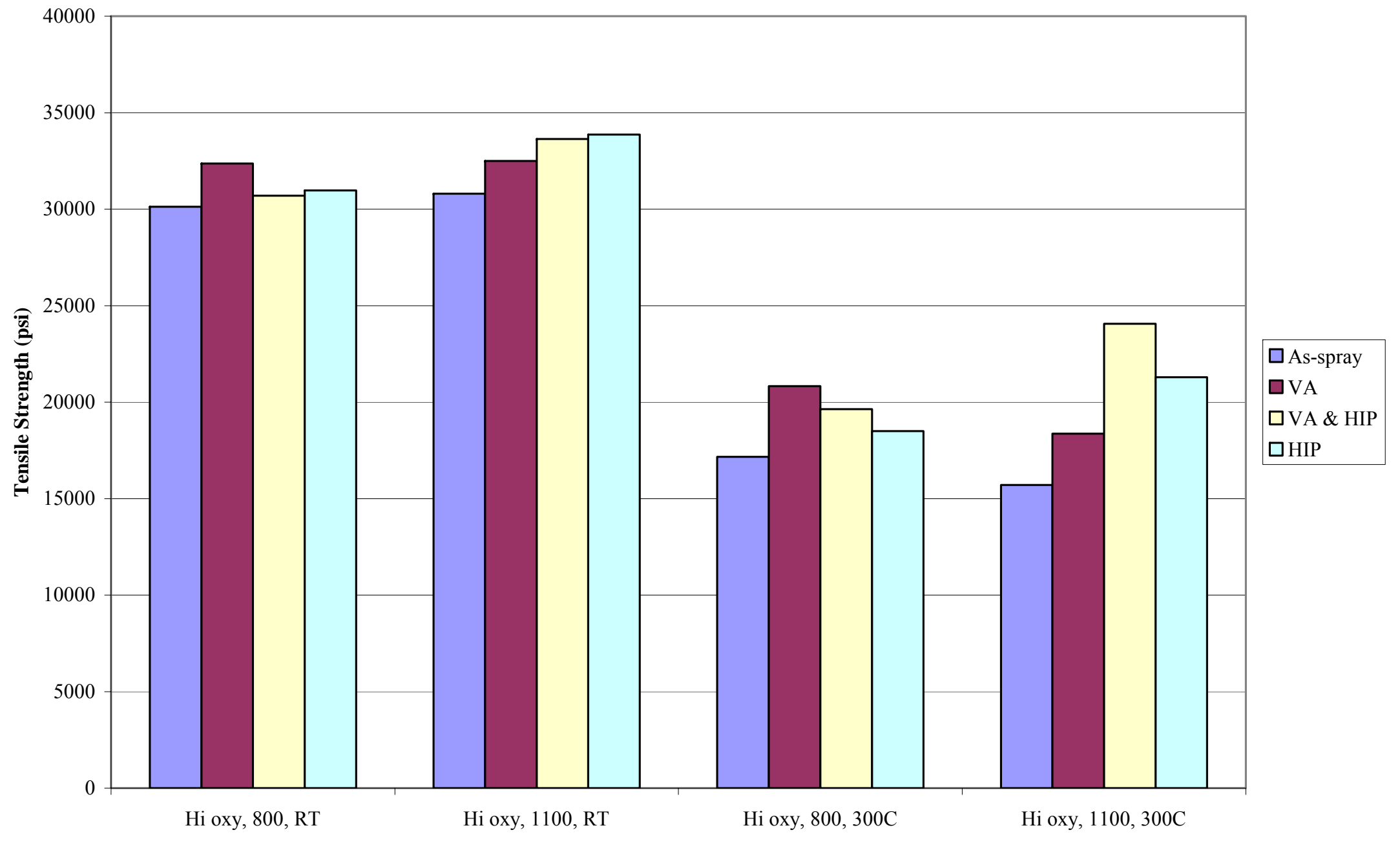

Figure 33 - Plot showing room temperature and elevated temperature ultimate tensile strength of VPS copper in different conditions produced using Hi oxy feedstock powder as a function of preheat temperature. 
Figures $34-37$ show the effect of feedstock powder and preheat temperature on the elongation of VPS copper deposits along with how post-spray heat treating effects elongation. Figure 34 plots the room temperature elongation for the different sample conditions as a function of feedstock powder and preheat temperature. Initial examine of the data shows little effect of preheat temperature on the elongation of the samples. However, oxygen content in the feedstock powder did show a significant effect on the elongation. For example, the Lo oxy feedstock powder samples had room temperature elongations that varied from $49-56 \%$. For comparison, the Hi oxy feedstock powder samples had room temperature elongations that varied from 40$53 \%$. Although all the post-spray heat treated samples had higher elongations as compared to their as-sprayed counterparts, the improvements in elongation were greater for the Hi oxy feedstock samples. However, even with this improvement from the post-spray heat treatments, the Lo oxy feedstock samples had the highest elongation values.

Figure 35 plots the elevated temperature elongation for the different sample conditions as a function of feedstock powder and preheat temperature. Similar to the room temperature elongation values, the samples produced with the Lo oxy feedstock powder had the highest elongation. The as-sprayed elevated temperature elongation values for the Lo oxy samples varied from $43-45 \%$. In contrast, the as-sprayed elevated temperature elongation values for the Hi oxy samples were significantly lower, i.e., $17-18 \%$. Post-spray heat treating did increase the elongation values for both the Lo and Hi oxy samples. However, even with post-spray heat treating, the samples produced with the Hi oxy feedstock powder had elevated temperature elongation values less than the as-sprayed elevated temperature elongation values for the samples produced with the Lo oxy feedstock powder.

Figure 36 plots the room and elevated temperature elongation values for the samples produced with the Lo oxy feedstock powder as a function of preheat temperature. The plot shows there is little effect of the preheat temperature on elongation. In addition, this figure shows with the proper post-spray heat treatment and using Lo oxy copper feedstock powder little degradation in percent elongation is possible from room temperature to elevated temperatures. In contrast, Figure 37 shows samples produced with Hi oxy copper feedstock powders are susceptible to significant reductions in elevated temperature percent elongations, even with post-spray heat treatments. 


\section{VPS Copper Elongation (RT)}

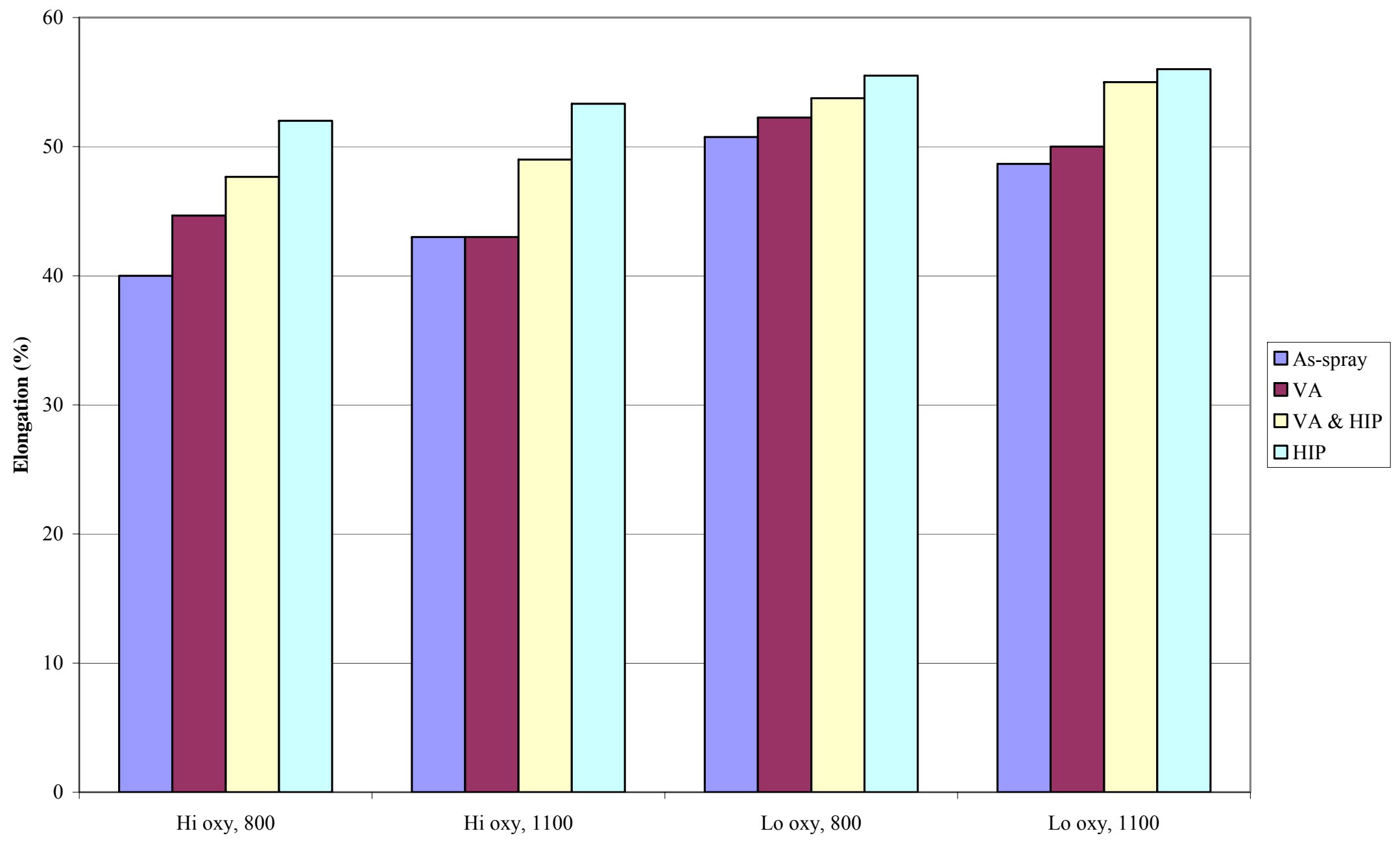

Figure 34 - Plot showing room temperature elongation of VPS copper in different conditions as a function of feedstock powder and preheat temperature. 


\section{VPS Copper Elongation (300C)}

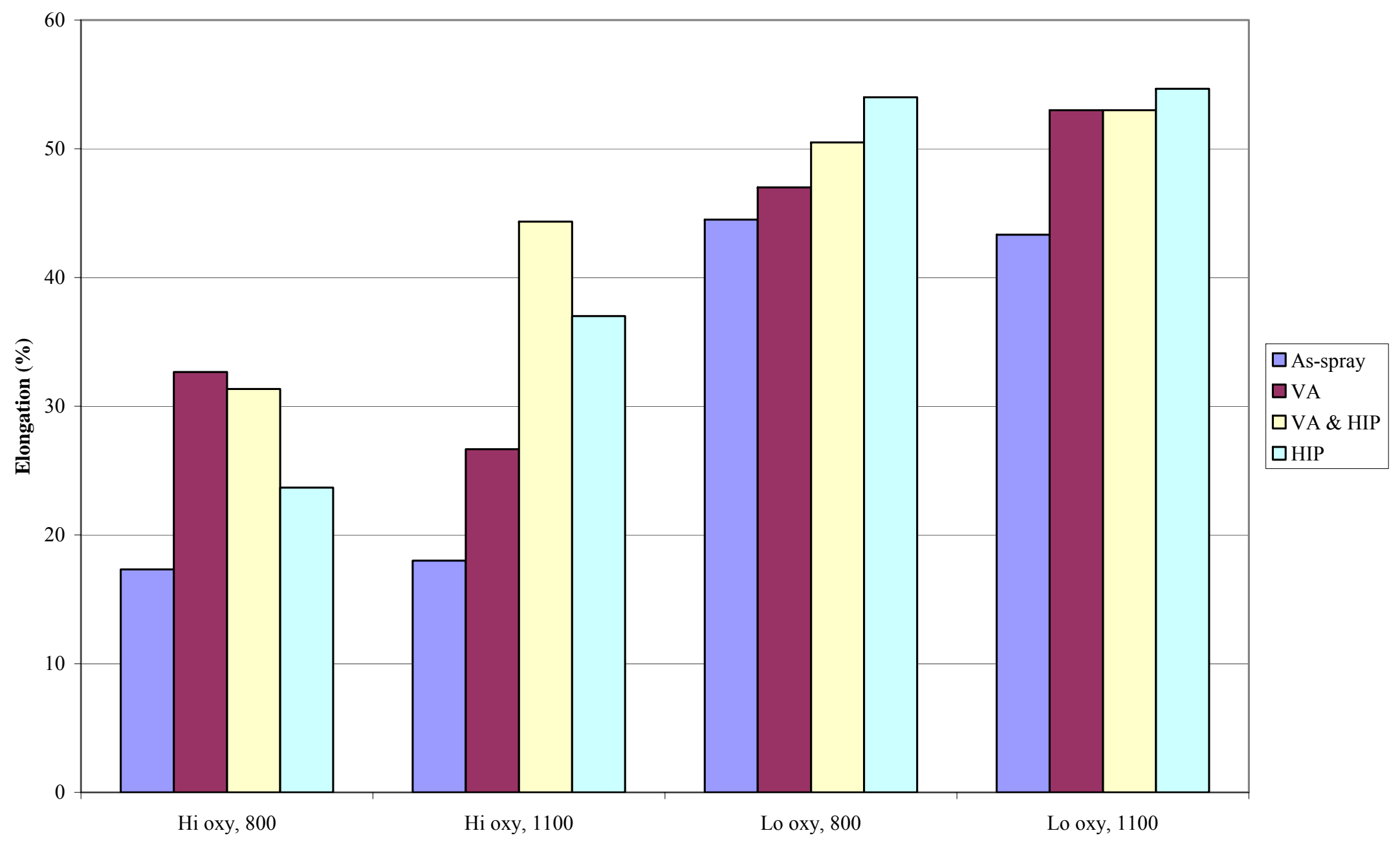

Figure 35 - Plot showing elevated temperature elongation of VPS copper in different conditions as a function of feedstock powder and preheat temperature. 
VPS Copper Elongation (Lo oxy)

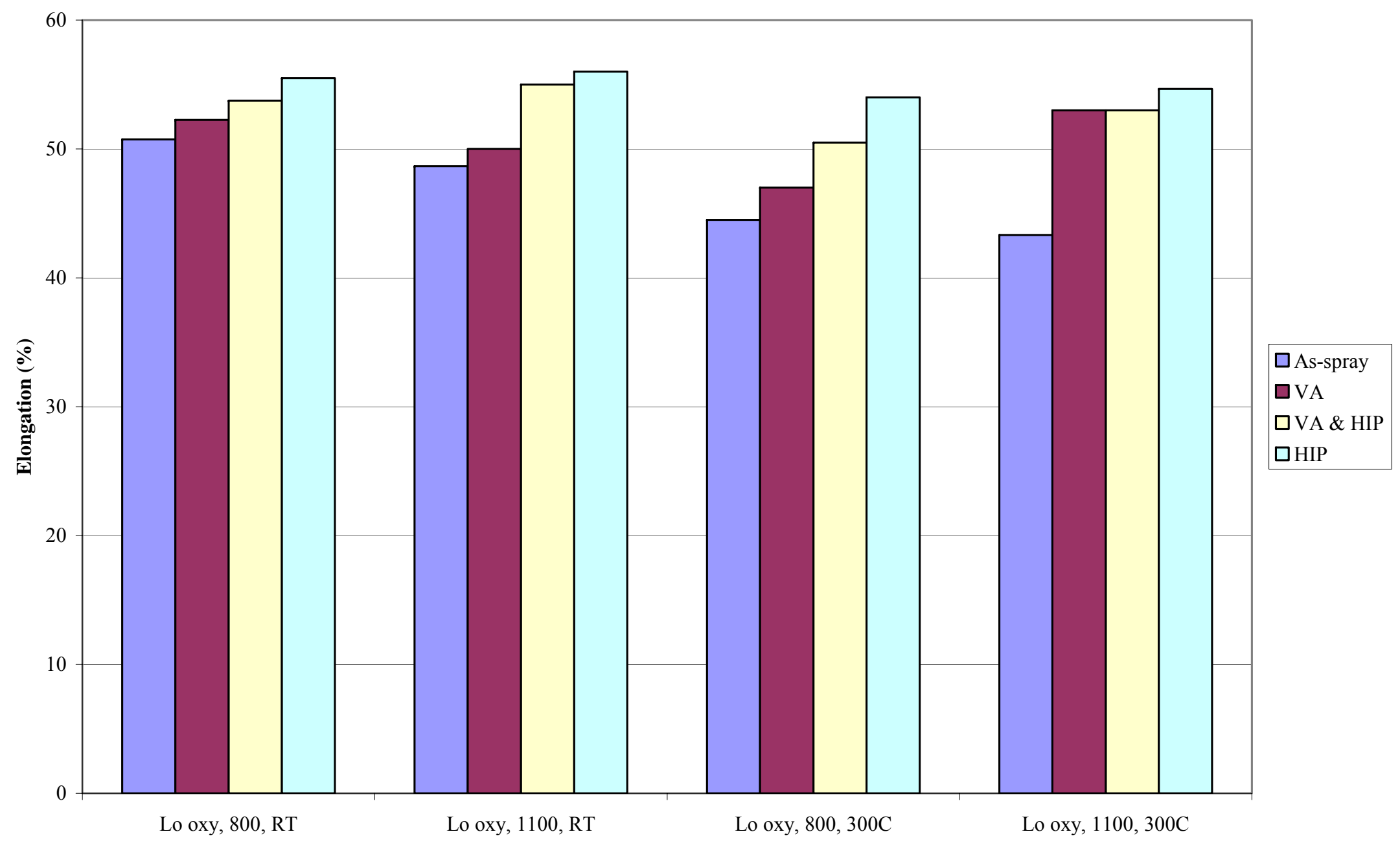

Figure 36 - Plot showing room temperature and elevated elongation of VPS copper in different conditions produced with Lo oxy feedstock powder as a function of preheat temperature. 


\section{VPS Copper Elongation (Hi oxy)}

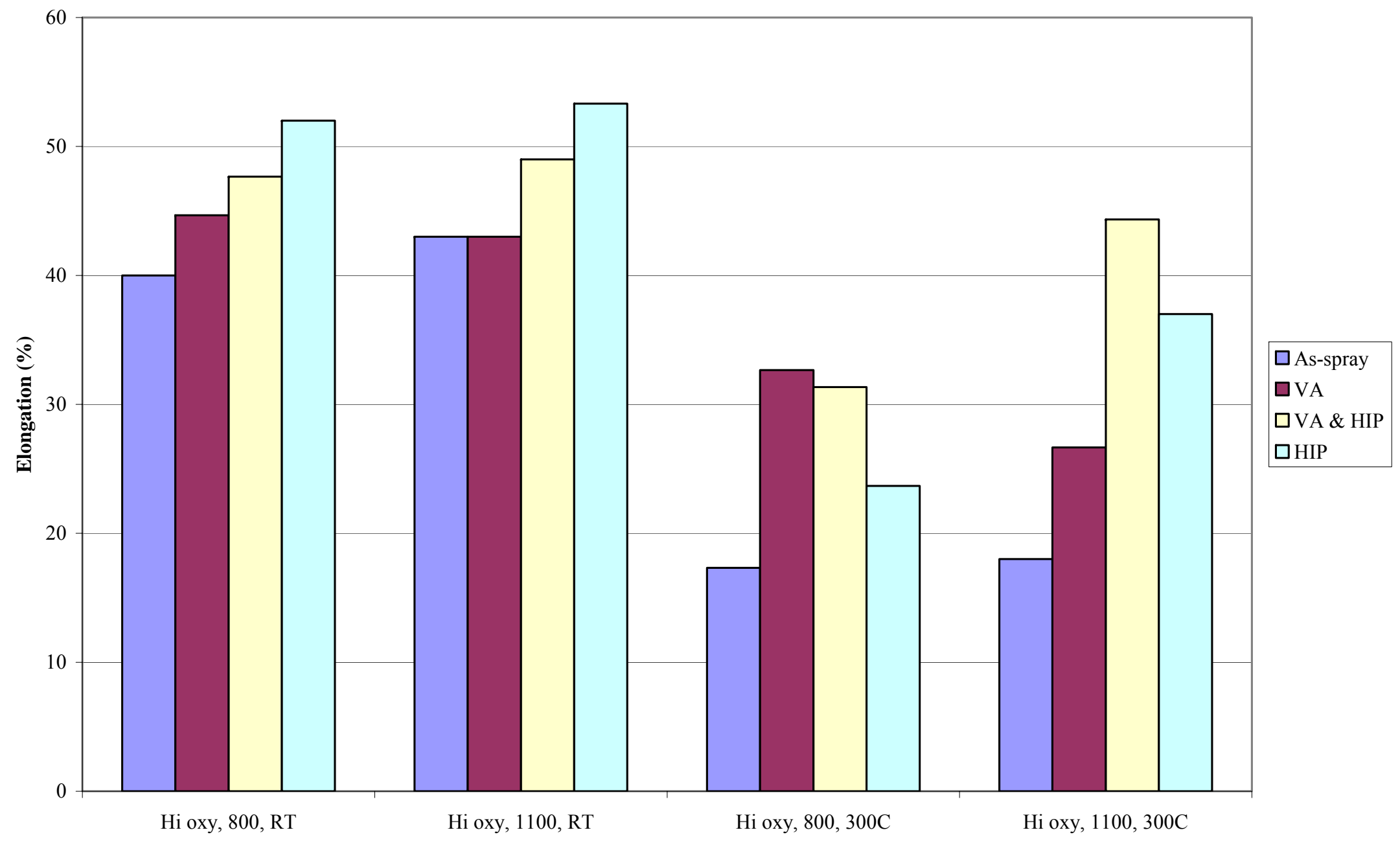

Figure 37 - Plot showing room temperature and elevated elongation of VPS copper in different conditions produced with Hi oxy feedstock powder as a function of preheat temperature. 


\subsubsection{VPS Copper Physical Properties Results and Discussion}

Table 10 lists the results of water immersion density measurements for VPS copper deposits produced with Lo oxy feedstock powder. Similar to the tensile results, two different preheat temperatures and three post-spray heat treatments were evaluated to determine their effect on density. Figure 38 shows a plot of the densities as a function of sample condition and preheat temperature. Note all the samples had a density greater than $97 \%$ of theoretical. However, the VA+HIP and HIP samples had density values of $99 \%$ of theoretical or greater. There was no significant difference between the densities for the two different preheat temperatures.

Table 10 - Density of VPS copper in as-sprayed and heat treated conditions.

\begin{tabular}{|c|c|c|}
\hline \multirow{2}{*}{ Condition } & \multicolumn{2}{|c|}{ Density $(\%$ of Theoretical $)$} \\
\cline { 2 - 3 } & $800^{\circ} \mathrm{C}$ Preheat & $1100^{\circ} \mathrm{C}$ Preheat \\
\hline As-Spray & 98.7 & 97.7 \\
\hline VA & 98.6 & 97.6 \\
\hline VA + HIP & 99.0 & 99.9 \\
\hline HIP & 100 & 99.8 \\
\hline
\end{tabular}

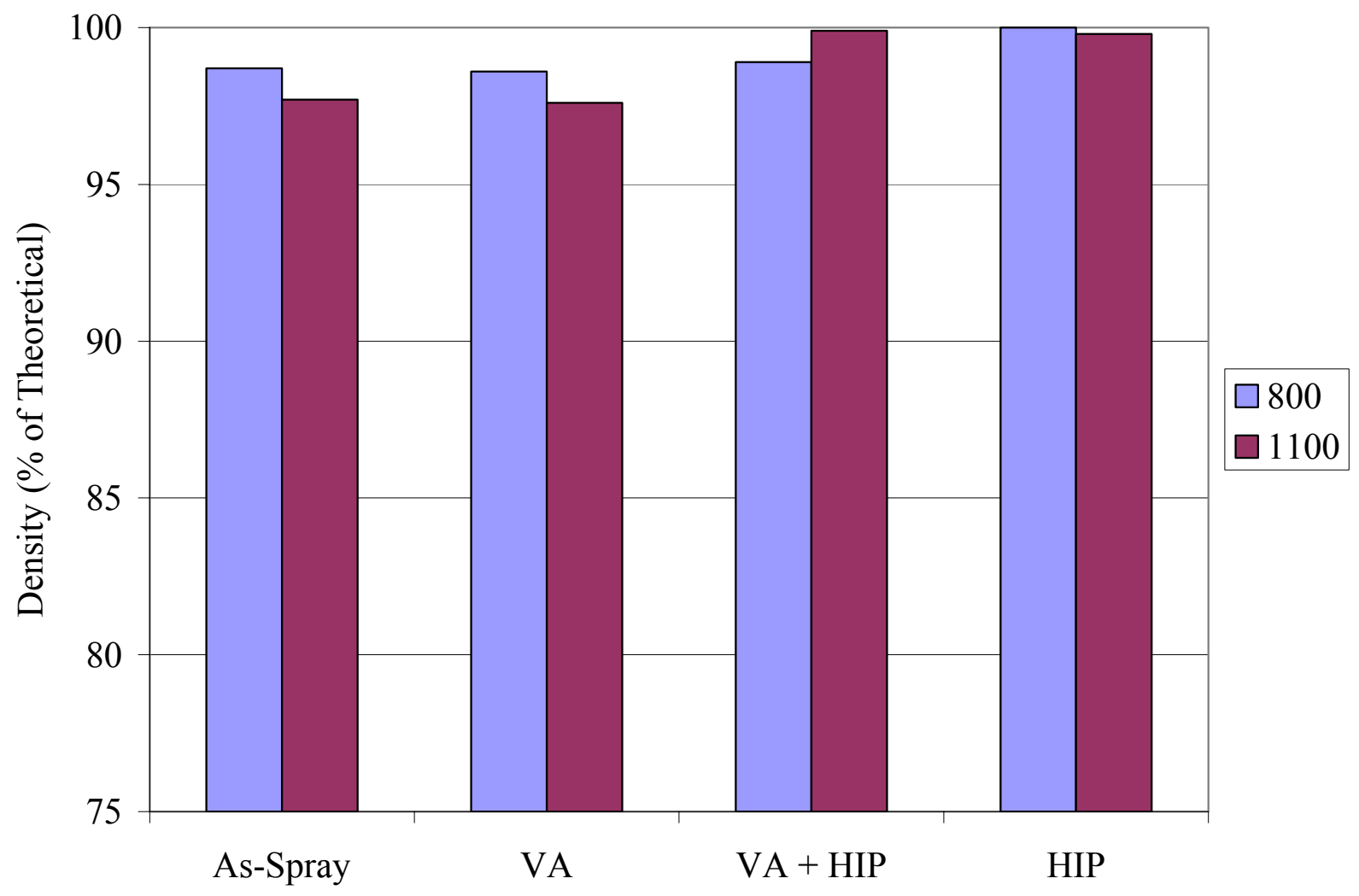

Figure 38 - Plot showing the densities of VPS Cu deposits in the as-sprayed and heat treated conditions. Note two samples with different preheat temperatures $\left(800\right.$ and $\left.1100^{\circ} \mathrm{C}\right)$ were tested for each condition. 
Figure 39 shows the VPS copper diffusivity results from the laser flash tests. Again, samples were tested in the as-sprayed, VA, VA+HIP, and HIP conditions, and two preheat temperatures were used to make the samples. This figure shows the room temperature diffusivity values ranges from $\sim 0.9$ to $1 \mathrm{~cm}^{2} / \mathrm{s}$. As the test temperature increased to $200^{\circ} \mathrm{C}$, the diffusivity values of the samples decreased. However from 200 to $300^{\circ} \mathrm{C}$, an $\sim 10 \%$ increase in the diffusivity value was noted for each sample. After $300^{\circ} \mathrm{C}$, the diffusivity values continued to decrease with increasing temperature. After the final measurement was made at $1000^{\circ} \mathrm{C}$ for each sample, the sample was allowed to cool to room temperature and another measurement was taken. This second room temperature measurement resulted in an $\sim 10 \%$ increase over the initial room temperature diffusivity measurement for each sample. It is believed this effect was due to residual stresses in the sample as a result of machining, and that after heating to $300^{\circ} \mathrm{C}$ the sample was annealed; thus, relieving the residual machining stresses. Regarding the effect of preheat temperature on the diffusivity, no significant difference was observed.

The density measurements and the diffusivities along with theoretical values for specific heat were then used to calculate the thermal conductivity of the VPS copper samples. These results are shown in Figure 40. Similar to the diffusivity results, the thermal conductivity values decrease with increasing temperature to $200^{\circ} \mathrm{C}$. From 200 to $300^{\circ} \mathrm{C}$ an $\sim 10 \%$ in thermal conductivity was observed for all samples. After $300^{\circ} \mathrm{C}$, the thermal conductivity of the VPS Cu deposits decreased as the test temperature increased. For comparison, the theoretical conductivity of pure copper was also plotted as a function of temperature. Using the annealed thermal conductivity values, the room temperature thermal conductivity of VPS copper is approximately $87 \%$ of the thermal conductivity of pure copper. At $300^{\circ} \mathrm{C}$, the thermal conductivity of VPS copper is approximately $92 \%$ of pure copper.

\subsection{Thermal Conductivity of VPS Tungsten}

Figure 41 shows the thermal conductivity of VPS tungsten in the as-sprayed and heat treated conditions. After high temperature heating, the VPS tungsten deposit has a thermal conductivity equivalent to that of wrought tungsten. However, in the as-sprayed condition, the room temperature thermal conductivity of VPS tungsten is approximately $85 \%$ of the thermal conductivity of wrought tungsten. Although these results show VPS tungsten after high temperature heat treating can achieve the equivalent thermal conductivity of wrought tungsten, deposition of the tungsten on the copper heat sink will preclude the use of high temperature heat treatments. Even using the copper heat treatments outlined in the previous sections will be insufficient to improve the thermal conductivity above that of the as-sprayed condition. 


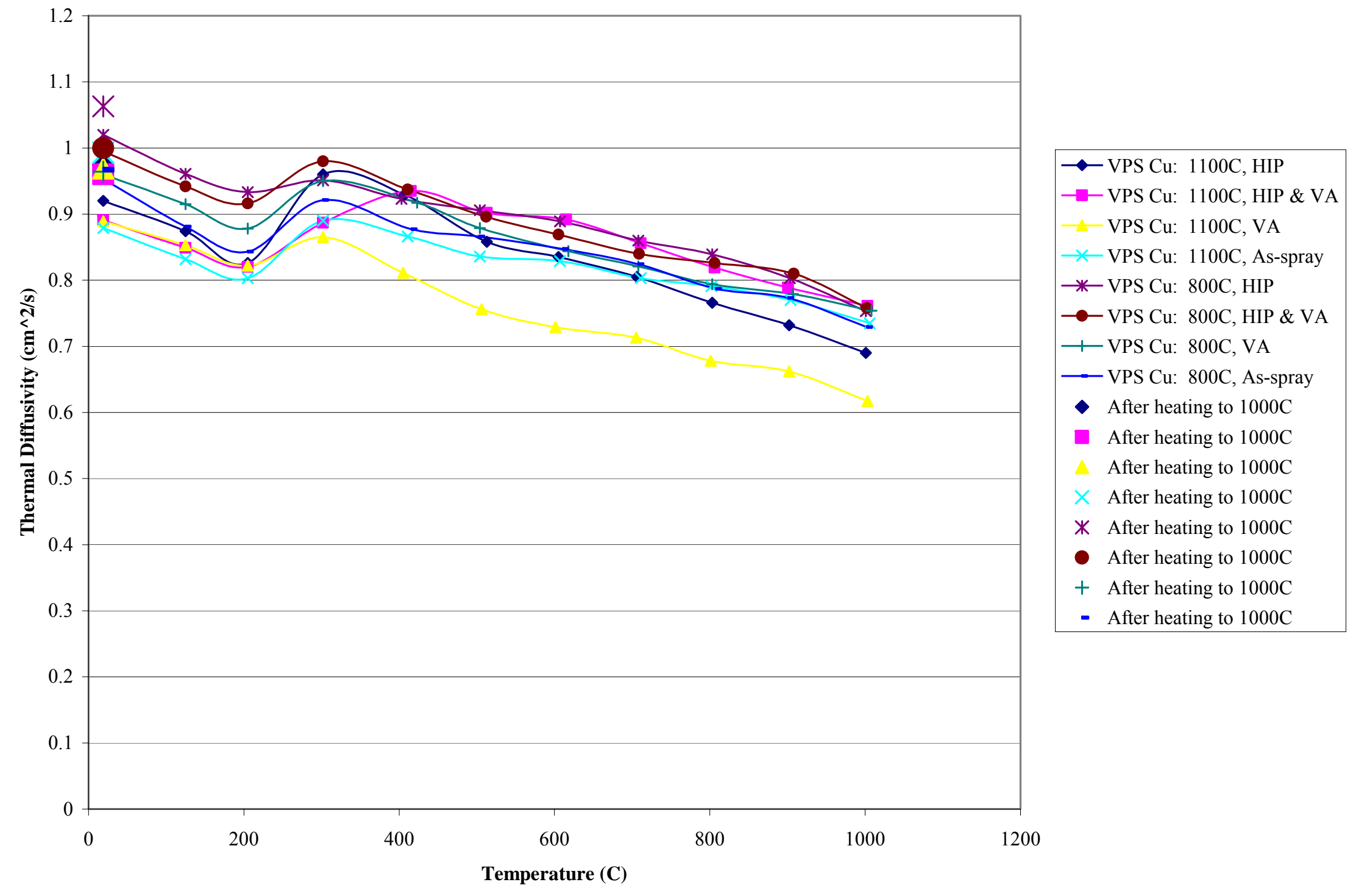

Figure 39 - VPS copper diffusivity as a function of temperature. 


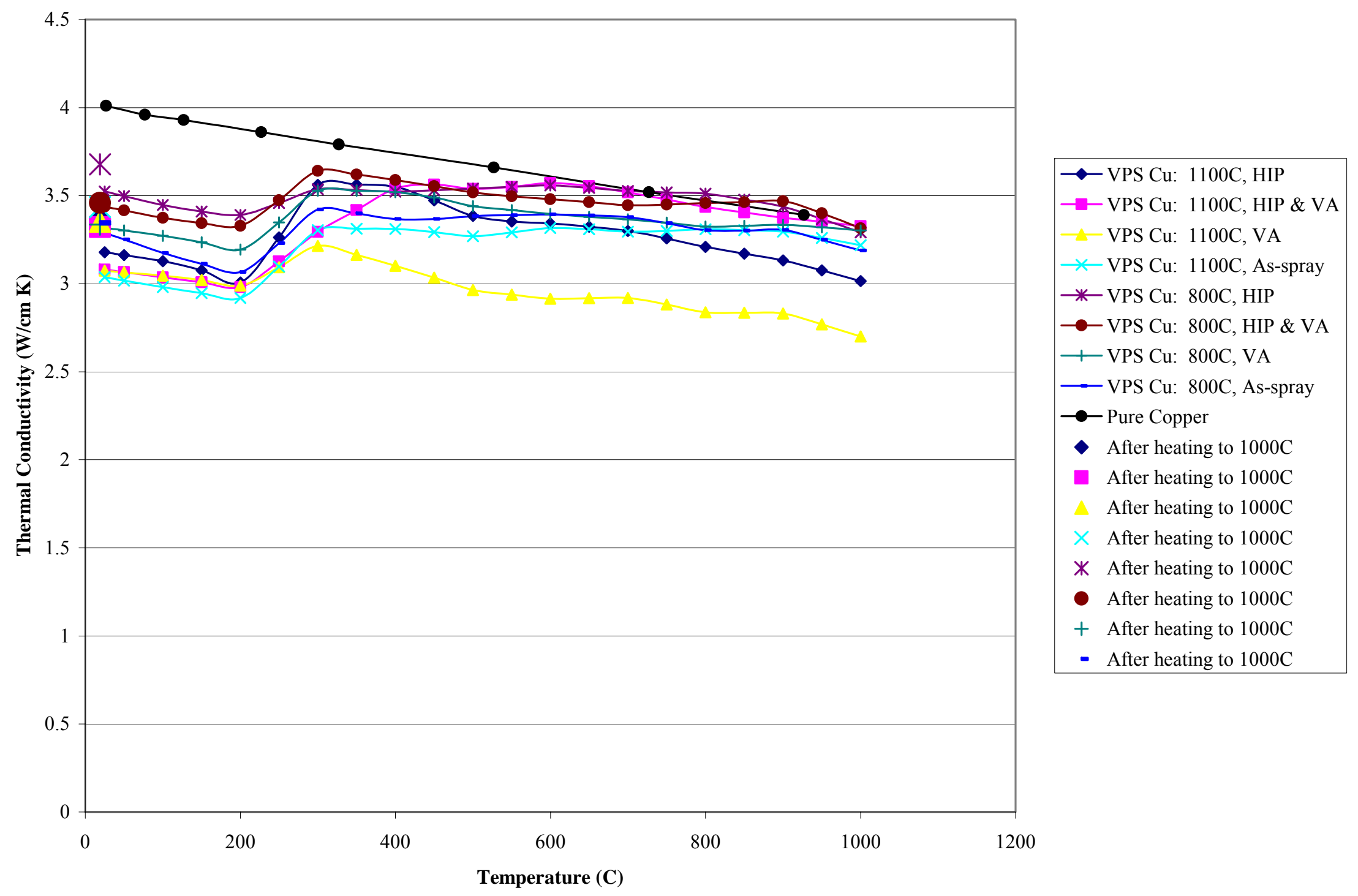

Figure 40 - VPS copper thermal conductivity as a function of temperature. 


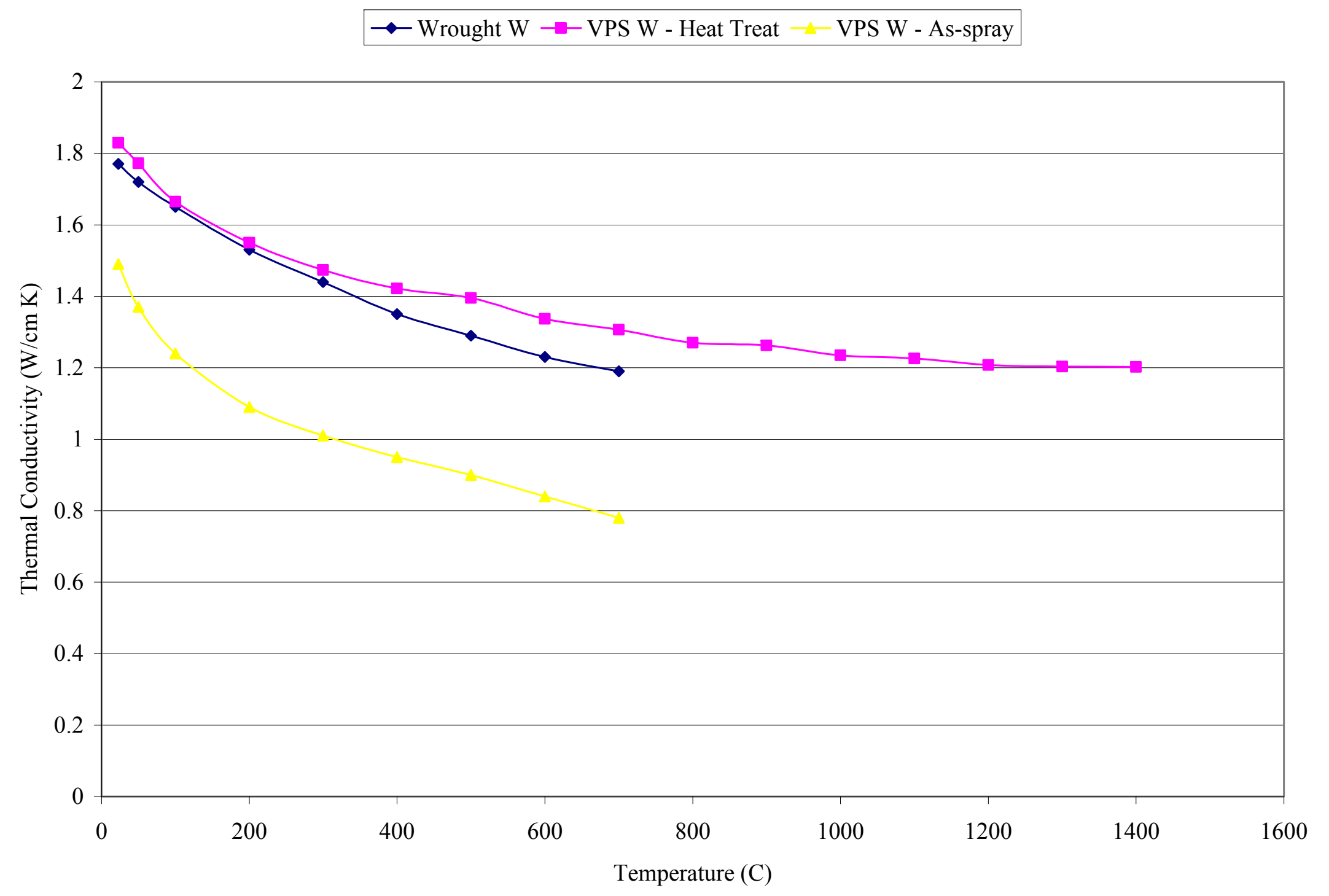

Figure 41 - Thermal conductivity of VPS tungsten in the as-sprayed and heat treated conditions as a function of temperature. 


\subsection{MEDIUM SCALE MOCKUP DEVELOPMENT}

During the Phase I investigation, small scale EBTS mockups were produced with $\sim 5 \mathrm{~mm}$ of VPS tungsten armor. A photograph of one of the EBTS mockups after trimming of the sides of the mockup using electric discharge machining (EDM) is shown in Figure 42. A $0.7 \mathrm{~mm}$ thick $\mathrm{Cu} / \mathrm{W}$ functional gradient was used to join the tungsten armor to the copper alloy heat sink (see Figure 43). A close-up view of the interface between the VPS deposited material and the heat sink reveals debonding of the armor from the heat sink (see Figure 44). The Phase I mockups were produced prior to the finite element modeling and process development efforts discussed in Sections 2 and 3 of this report. Therefore, based on the previous experience from the Phase I effort and the results of the modeling effort, a $2 \mathrm{~mm} \mathrm{Cu}$-rich gradient was selected for producing the medium scale mockups during the Phase II investigation.

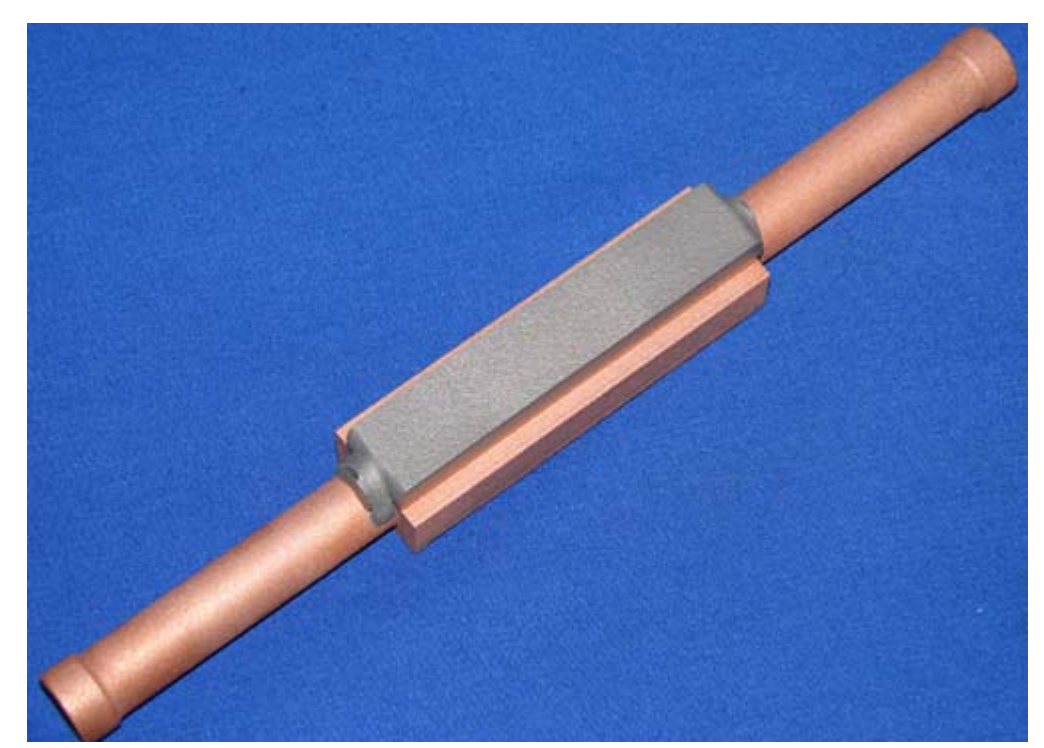

Figure 42 - Small scale EBTS mockup produced during the Phase I investigation.

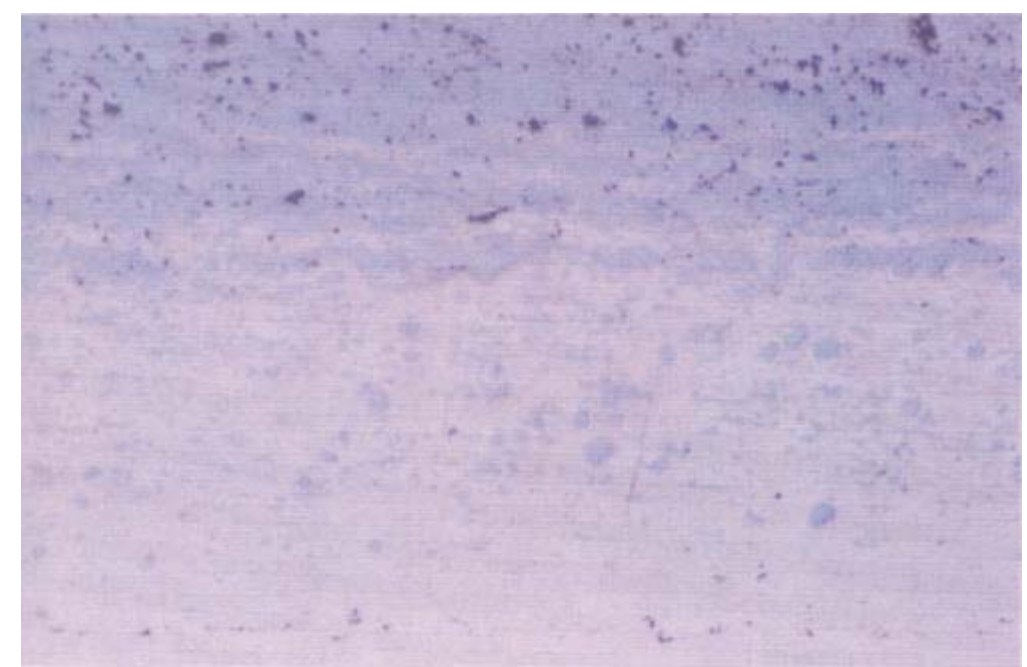

Figure 43- Non-optimized functional gradient used during the Phase I investigation. Gradient thickness was approximately $0.7 \mathrm{~mm}$. 


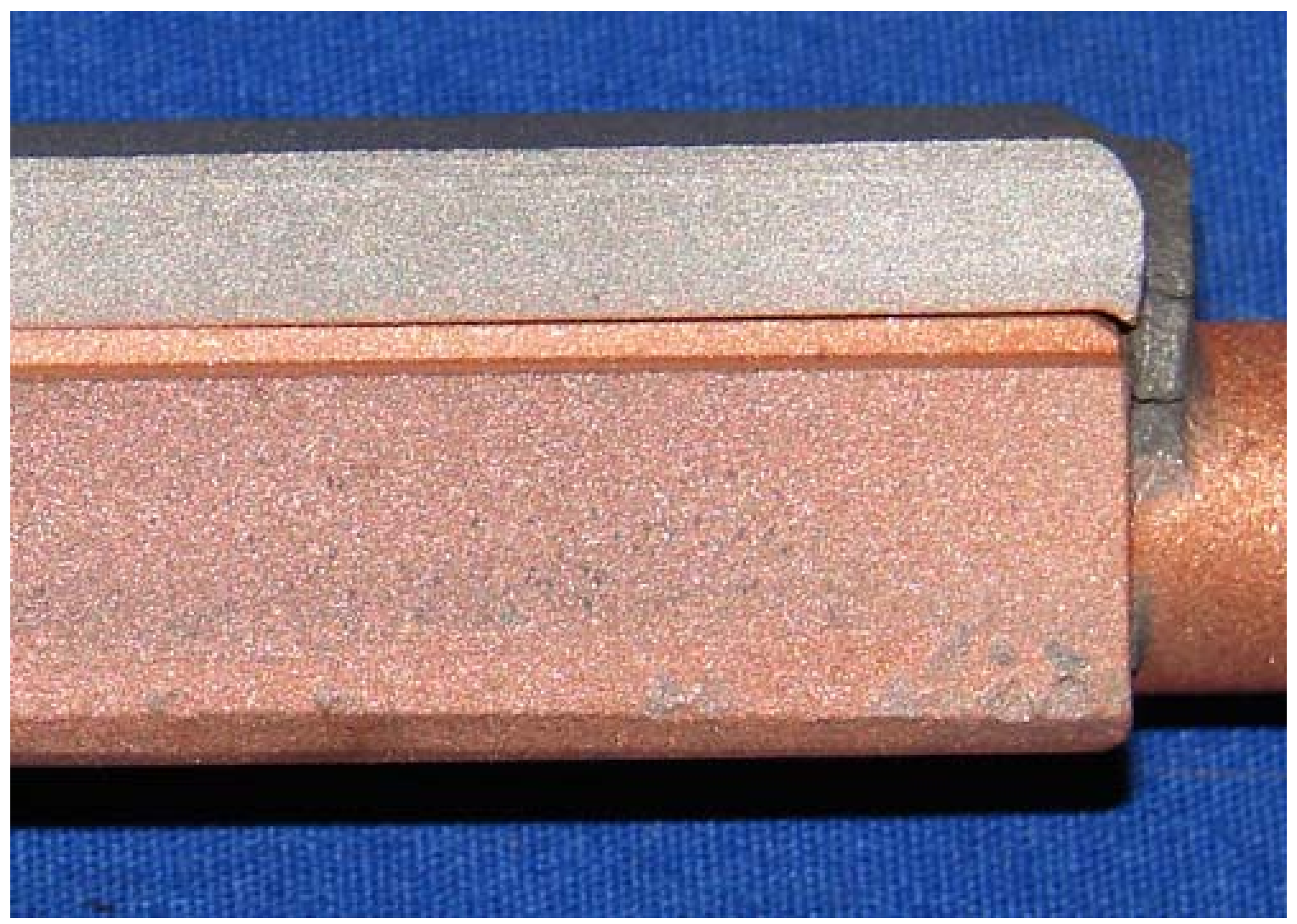

Figure 44 - Close-up view of the interface between the copper alloy heat sink and the VPS deposited material after electric discharge machining. Note the debond that was found at the interface.

\subsection{Medium Scale Mockup Design}

A drawing of the CuCrZr heat sink used for the medium scale mockups is shown in Figure 45. Note the medium scale heat sink has dual cooling channels as opposed to the EBTS mockup which is a single channel design. The overall length of the medium scale mockup is $400 \mathrm{~mm}$ and the width is $40 \mathrm{~mm}$ in the untrimmed condition. After deposition of the armor, the width is trimmed to $32 \mathrm{~mm}$. Similar to the EBTS mockup, the diameter of the cooling channels for the medium scale mockup is $10 \mathrm{~mm}$ each. 


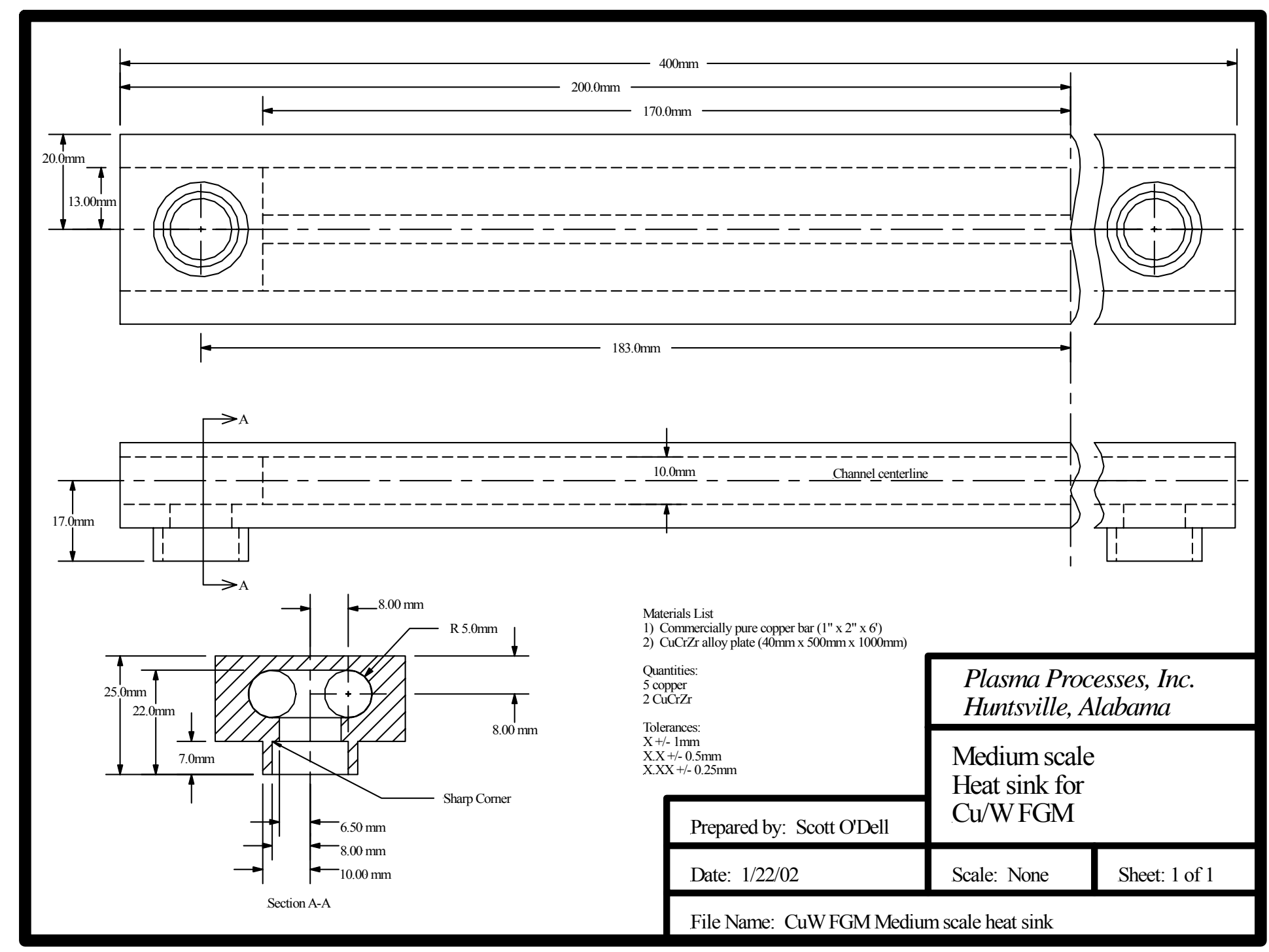

Figure 45 - Drawing of the medium scale, dual cooling channel $\mathrm{CuCrZr}$ heat sink. 


\subsection{VPS Joining and Armor Deposition}

During this initial stage of the investigation, vacuum plasma spray parameters were developed for producing dense deposits of $\mathrm{Cu} / \mathrm{W}$ FGMs and thick tungsten deposits. PPI's patented, parallel flow, bell contoured nozzles were used during this research to reduce overspray and produce dense deposits. The tungsten used during vacuum plasma spraying was $>99.6 \%$ pure tungsten crystalline powder with a mesh size of -325 . Oxygen free, high conductivity (OFHC) copper which was $99.9 \%$ pure, mesh size of -325 and had an oxygen content of les than 1000 parts per million (ppm) was used in the fabrication of the $\mathrm{Cu}$-rich functional gradient joint. In addition, a bondcoat comprised of an elemental blend of copper and nickel was used to promote diffusion between the deposited material and the copper alloy substrate. As a result, a metallurgical joint was created as compared to a mechanical joint, which is typical for most thermal sprayed coatings. A typical spray procedure used to produce the $\mathrm{Cu} / \mathrm{W}$ functional gradient joint and tungsten armor is showing in Appendix B.

Figure 46 shows an overall view of one of the medium scale mockups after VPS deposition. This figure shows the plasma gun used during spraying and the attached power cables. Two powder feed liners are also shown in the photograph. These lines allow feeding of the powder directly into the nozzle for optimum melting. Both the part and the gun moved during deposition with the gun moving in a direction perpendicular to the direction of the part. As a result, a rectilinear pattern was used during plasma spraying.

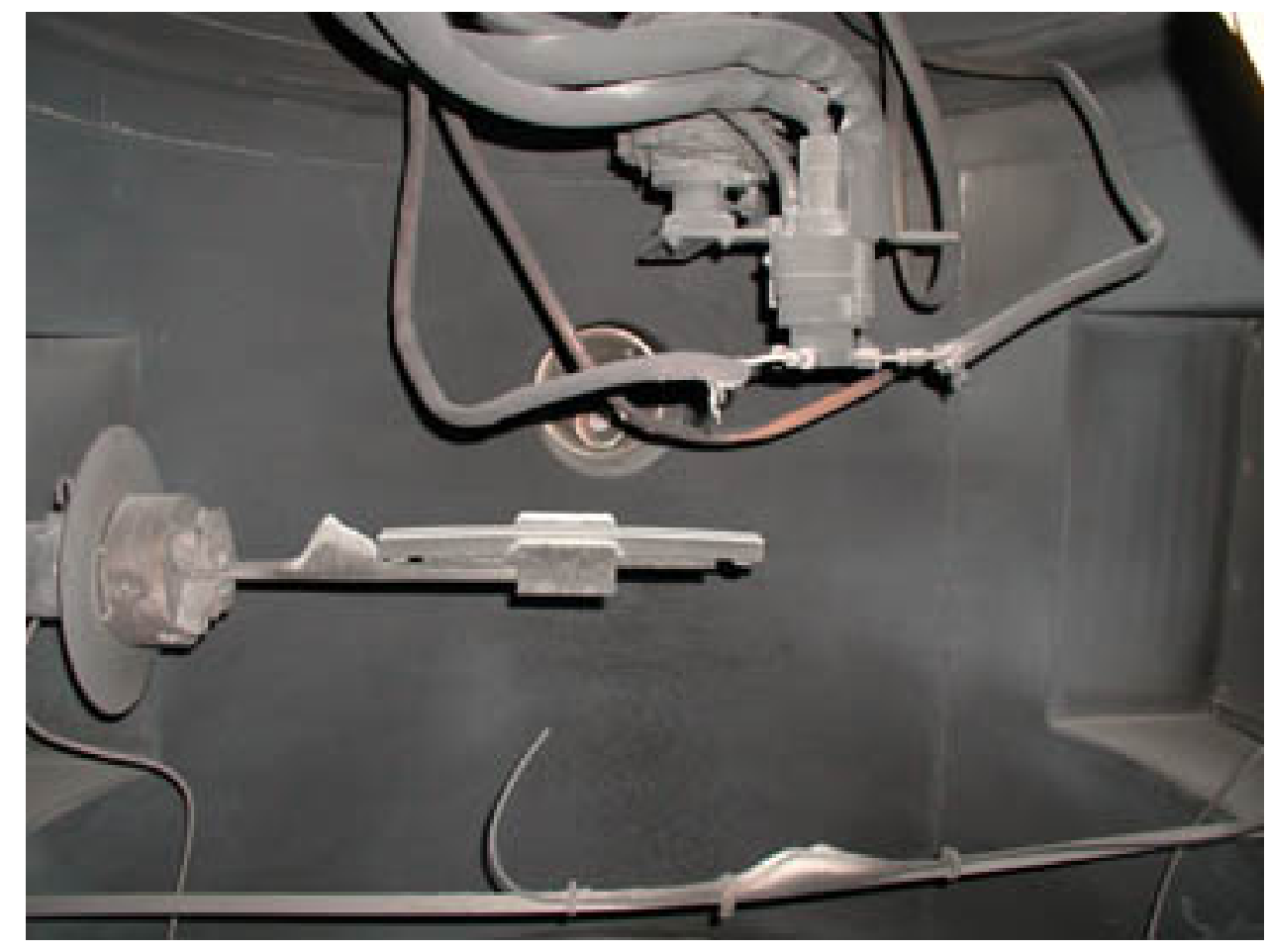

Figure 46 - Photograph showing a medium scale mockup inside the vacuum plasma spray chamber after deposition. 
Prior to spraying, the gun without powder being fed was used to preheat the copper heat sink. Figure 47 shows a copper alloy heat sink during preheat. Note without powder flowing, the plasma flame has a bright pink tint. Figure 48 shows the flame as powder is being fed. Note the flame color changed to bright green, which is typical for copper. Bright orange copper particles can be seen coming from the flame near its tip, just prior to depositing on the copper alloy heat sink. After cooling to room temperature, the coated heat sink was removed from the chamber. Electric discharge machining techniques were then used to trim the sides of the mockup and to cut castellations. Thermocouple holes were then drilled into the copper heat sink. Drawings showing the EDM cuts and hole positions are shown in Figures 49 and 50, respectively.

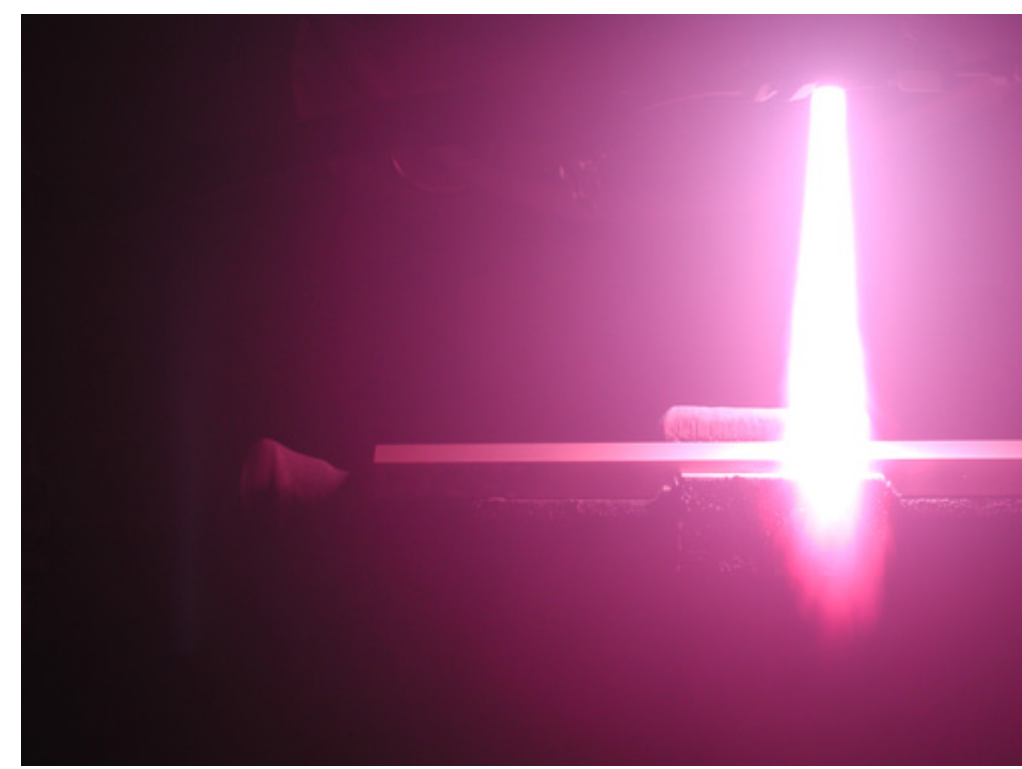

Figure 47 - Photograph showing plasma gun being used to preheat the $\mathrm{CuCrZr}$ heat sink.

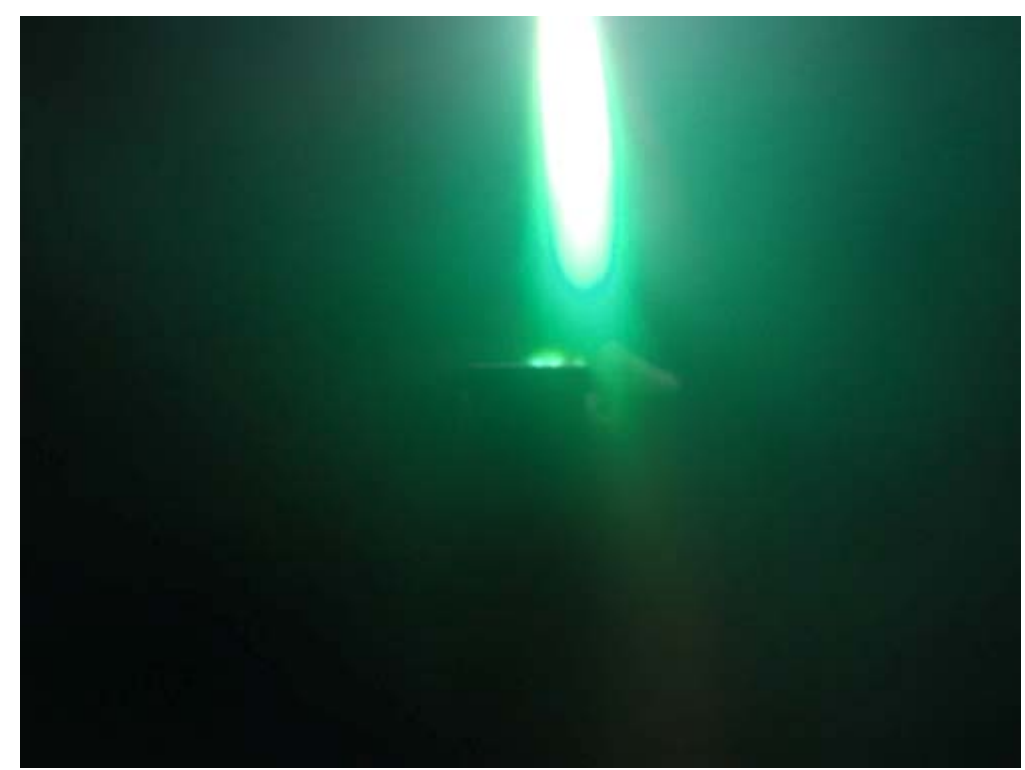

Figure 48 - Photograph showing deposition of Cu-rich gradient on the $\mathrm{CuCrZr}$ heat sink. 


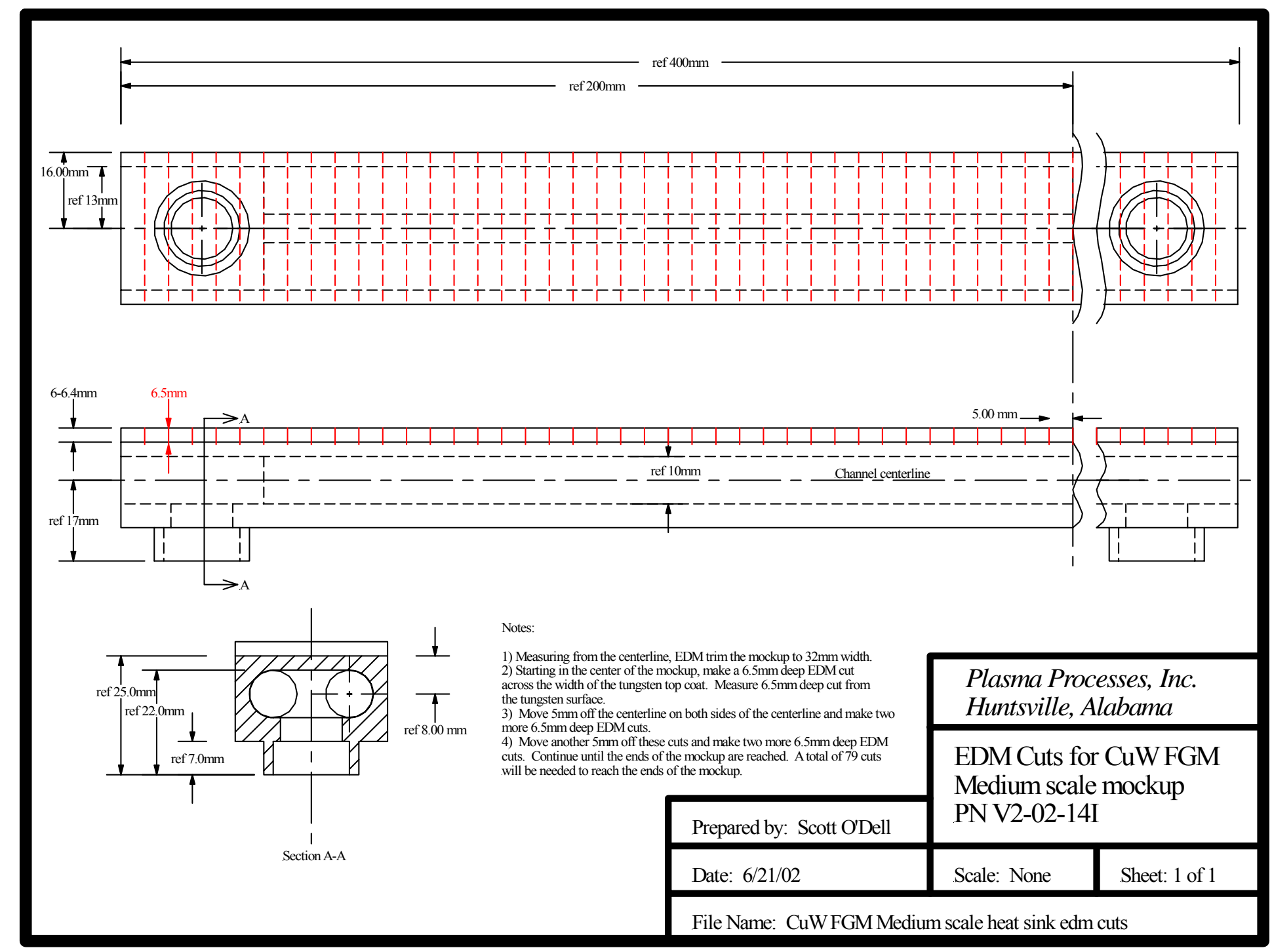

Figure 49 - Drawing showing medium scale mockup after EDM trimming of sides and tile breaks. 


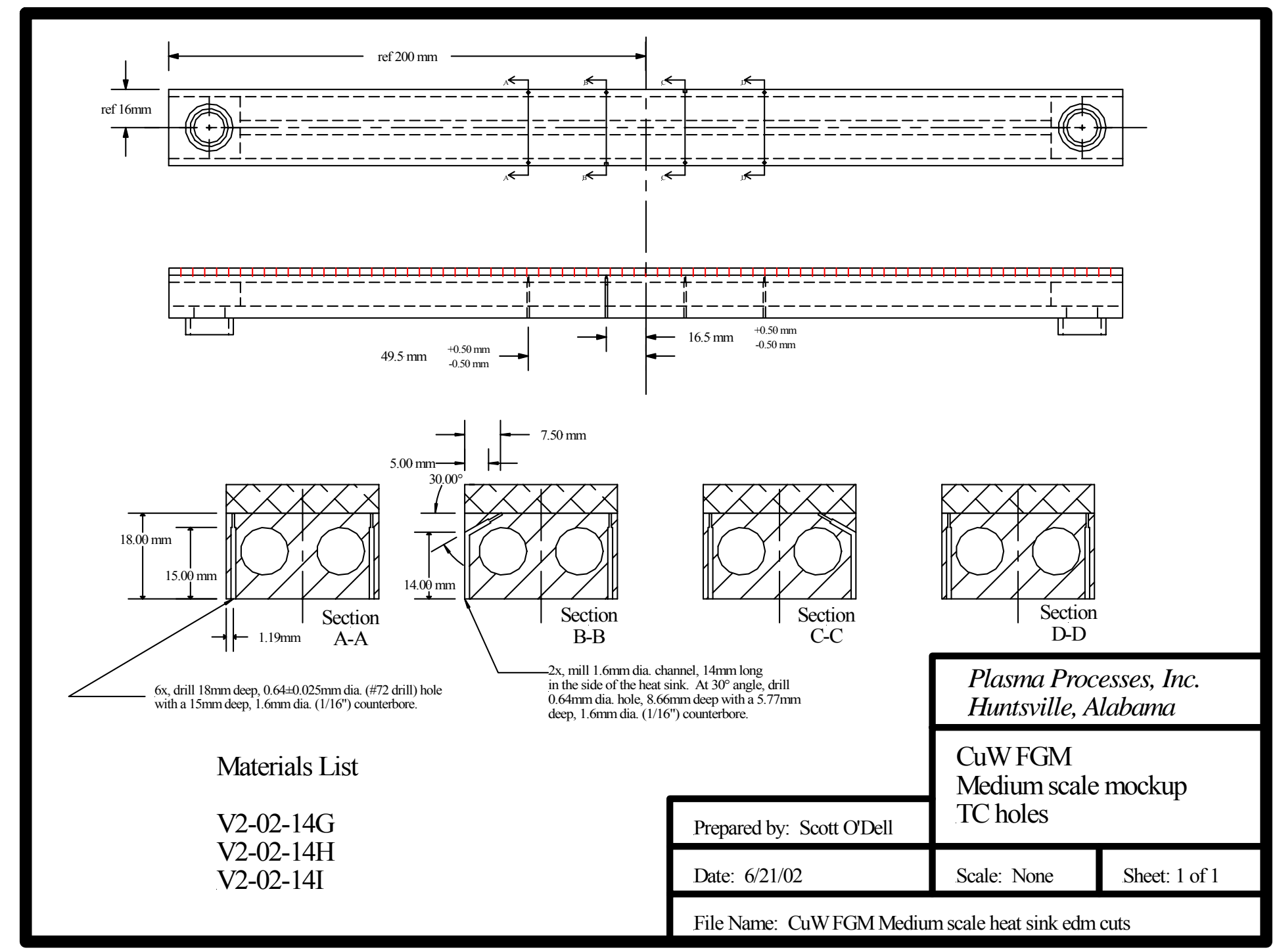

Figure 50 - Drawing of medium scale mockup showing thermocouple hole positions. 
Table 11 lists the three medium scale mockups produced during this investigation with a brief description for each mockup. Each of the three mockups used a $\mathrm{Cu}-\mathrm{Ni}$ bondcoat and a $2 \mathrm{~mm}$ thick $\mathrm{Cu}$-rich gradient. The tungsten armor thickness for the first mockup (V2-02-14G) was approximately $3 \mathrm{~mm}$. Excellent bonding was observed with a slight convex bow in the heat sink due to residual tensile stresses in the deposited material. These residual tensile stresses resulted in compressive stresses in the heat sink, which caused the slight bending.

For the final two medium scale mockups, a tungsten armor thickness of 5mm was achieved. Again, tensile stresses in the deposited material resulted in compressive stresses in the heat sinks causing the mockups to have convex bows. The bending in the $5 \mathrm{~mm}$ thick samples was slightly more than the bending in the $3 \mathrm{~mm}$ thick tungsten deposit. This was expected due to the increase in residual stress with increasing coating thickness. Of particular interest was the fact that no debonding of the VPS deposited material was observed for any of the mockups even with the bending.

Table 11 - Description of medium scale mockups after VPS deposition.

\begin{tabular}{|c|c|c|c|c|c|c|}
\hline \multirow[b]{2}{*}{ ID \# } & \multicolumn{2}{|c|}{ Heat Sink } & \multicolumn{3}{|c|}{ Vacuum Plasma Spray Material } & \multirow[b]{2}{*}{ Comments } \\
\hline & Material & Type & Bondcoat & Gradient & $\begin{array}{l}\text { Tungsten } \\
\text { Armor }\end{array}$ & \\
\hline V2-02-14G & $\mathrm{CuCrZr}$ & $\begin{array}{c}\text { Dual } \\
\text { channel }\end{array}$ & $\mathrm{Cu}-\mathrm{Ni}$ & $\begin{array}{l}\text { Cu-rich } \\
2 \mathrm{~mm}\end{array}$ & $\sim 3 \mathrm{~mm}$ & $\begin{array}{l}\text { Excellent bonding; Slight } \\
\text { bow in heat sink }\end{array}$ \\
\hline V2-02-14H & $\mathrm{CuCrZr}$ & $\begin{array}{c}\text { Dual } \\
\text { channel }\end{array}$ & $\mathrm{Cu}-\mathrm{Ni}$ & $\begin{array}{l}\text { Cu-rich } \\
2 \mathrm{~mm}\end{array}$ & $\sim 5 \mathrm{~mm}$ & $\begin{array}{l}\text { Excellent bonding; Slight } \\
\text { bow in heat sink }\end{array}$ \\
\hline V2-02-14I & $\mathrm{CuCrZr}$ & $\begin{array}{c}\text { Dual } \\
\text { channel }\end{array}$ & $\mathrm{Cu}-\mathrm{Ni}$ & $\begin{array}{l}\mathrm{Cu}-\mathrm{rich} \\
2 \mathrm{~mm}\end{array}$ & $\sim 5 \mathrm{~mm}$ & $\begin{array}{l}\text { Excellent bonding; Slight } \\
\text { bow in heat sink }\end{array}$ \\
\hline
\end{tabular}

Figure 51 shows a top view of the three mockups. Note the mockups are shown after machining of the tile breaks and trimming to a $32 \mathrm{~mm}$ width for each mockup. A brief description of the mockups after machining is given in Table 12. Machining of the castellations in V2-02-14G ( $3 \mathrm{~mm}$ thick tungsten armor) resulted in an elimination of the residual stresses from fabrication, and as a result, the bend that was present after fabrication was removed (see Figure 52). Similar reduction in stresses were observed for the thicker armored mockups (V2-02-14H and V2-0214I); however, a slight bend was still present in both mockups after castellating. Figure 53 shows a side view of the three mockups, where the slight bows in the two thick armored mockups are visible. 
Table 12 - Description of the medium scale mockups after machining of castellations.

\begin{tabular}{|c|c|c|c|}
\hline \multirow{2}{*}{ ID \# } & \multicolumn{2}{|c|}{ Armor Description } & \multirow{2}{*}{ Comments } \\
\hline & Thickness & Tile Width & \\
\hline V2-02-14G & $\sim 3 \mathrm{~mm}$ & $5 \mathrm{~mm}$ & $\begin{array}{l}\text { Castellation of the armor resulted in complete } \\
\text { elimination of the bow in the mockup that was } \\
\text { present after initial fabrication. }\end{array}$ \\
\hline V2-02-14H & $\sim 5 \mathrm{~mm}$ & $5 \mathrm{~mm}$ & $\begin{array}{l}\text { Castellation of the armor reduced the amount of } \\
\text { bend in the mockup, but did not eliminate it } \\
\text { completely. }\end{array}$ \\
\hline V2-02-14I & $\sim 5 \mathrm{~mm}$ & $5 \mathrm{~mm}$ & $\begin{array}{l}\text { Castellation of the armor reduced the amount of } \\
\text { bend in the mockup, but did not eliminate it } \\
\text { completely. }\end{array}$ \\
\hline
\end{tabular}

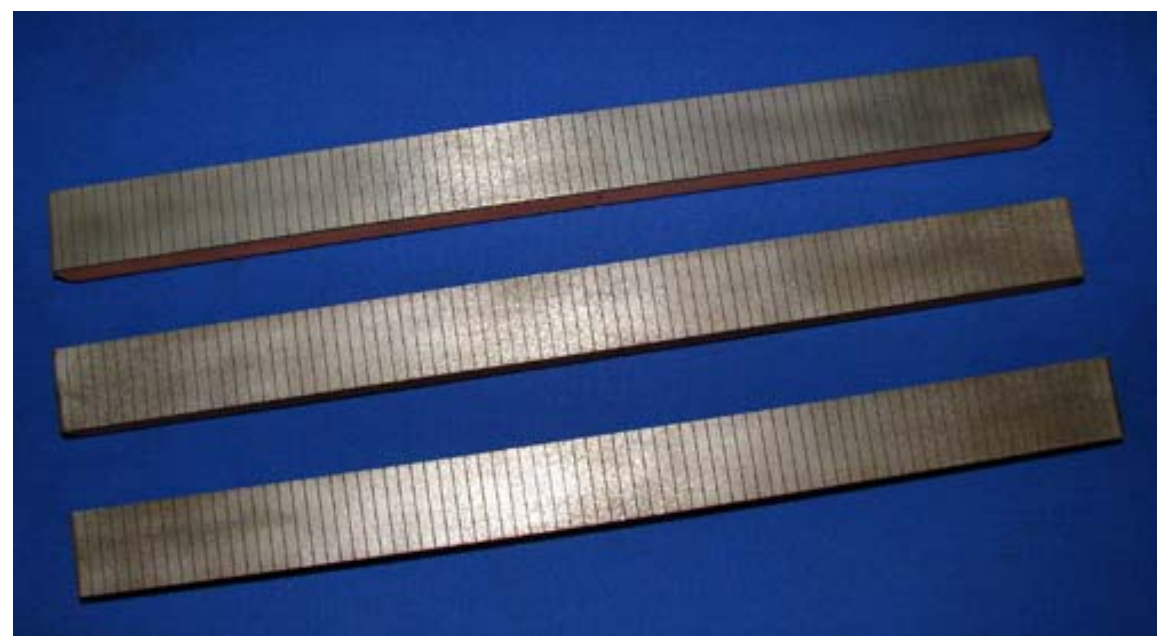

Figure 51 - Top view of the three medium scale mockups after machining of tile breaks.

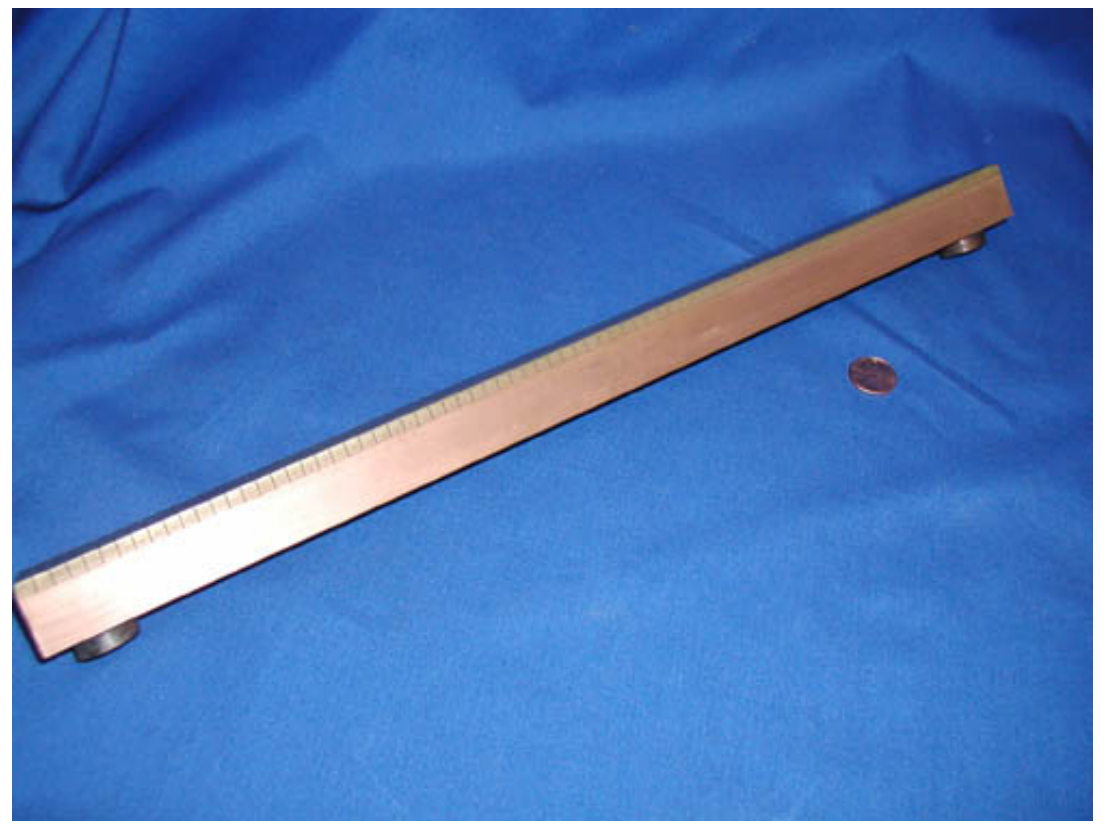

Figure 52 - Side view of sample V2-02-14G showing the elimination of the bow in the mockup as a result of machining the tile breaks. 


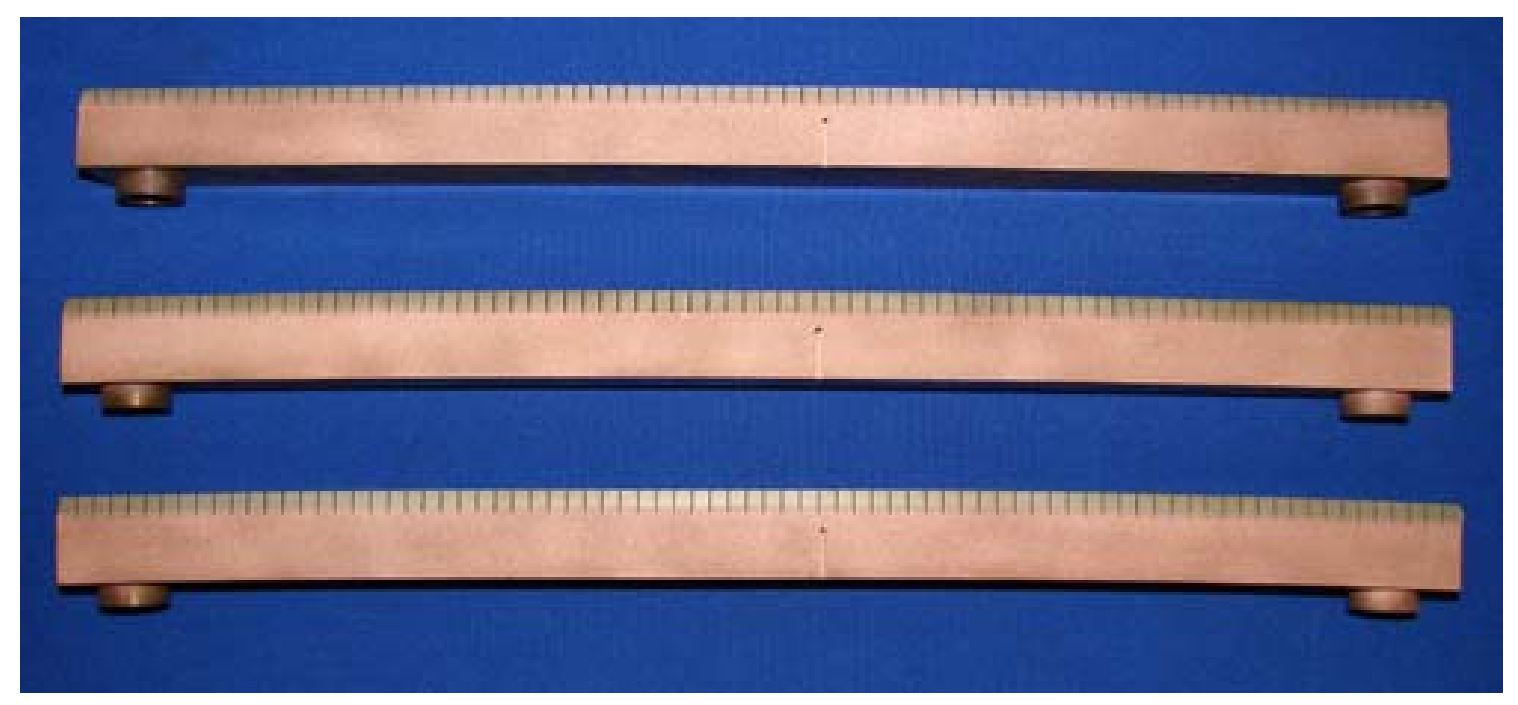

Figure 53 - Photograph showing the side view of all three mockups after machining of tile breaks. Samples from top to bottom are V2-02-14G, V2-02-14H, and V2-02-14I. Note even after machining of the tile breaks, the mockups with the thick tungsten armor (V2-02-14H and V2-02-14I) still have a small amount of bow in the heat sinks.

A close-up view of the castellated tungsten armor for mockup V2-02-14G is shown in Figure 54. This figure shows how the tile breaks extend just into the copper alloy heat sink. A photomicrograph showing the functional gradient joint formed between the heat sink and the tungsten armor is shown in Figure 55. Note the excellent bond achieved using the $\mathrm{Cu}-\mathrm{Ni}$ bonding agent, and the gradual transition of the functional gradient material from a $\mathrm{Cu}-\mathrm{rich}$ region to pure tungsten armor.

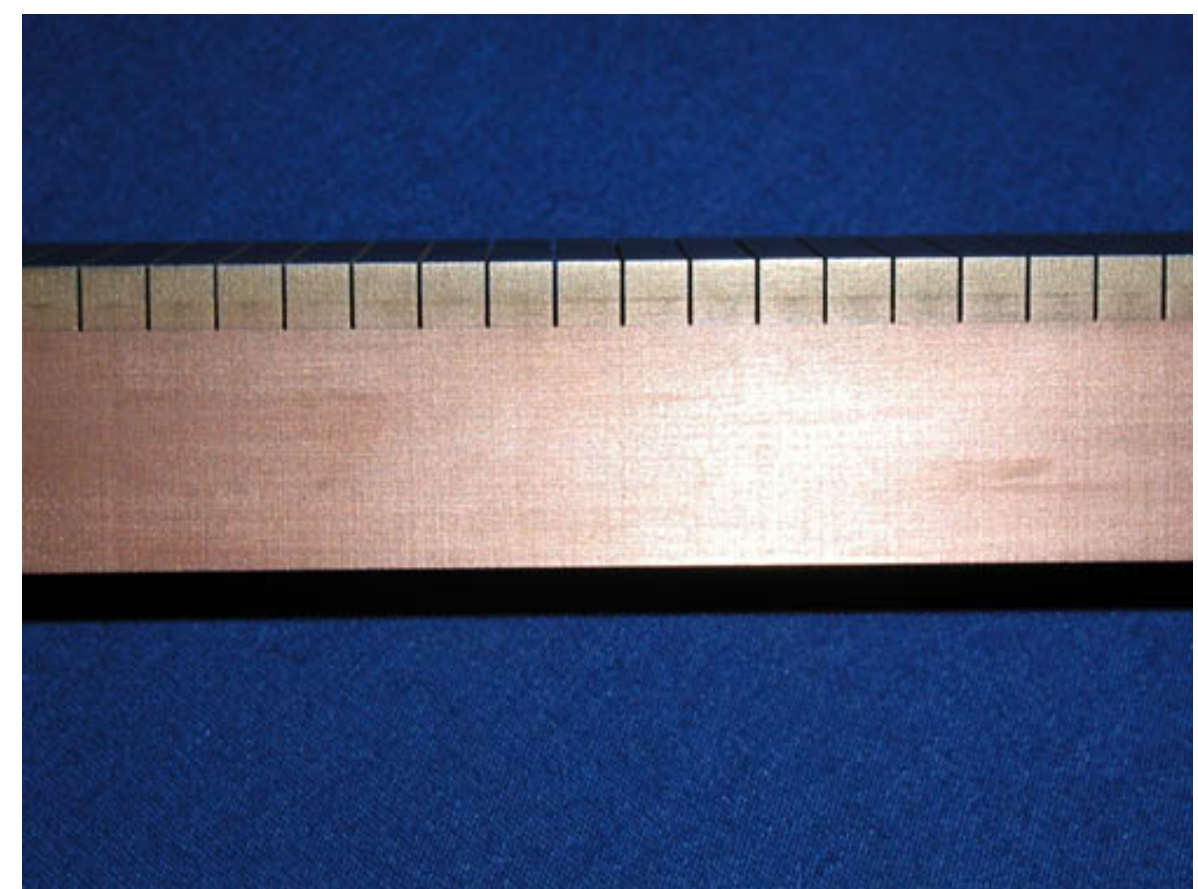

Figure 54 - Close-up view of castellated tungsten armor for sample V2-02-14G. 


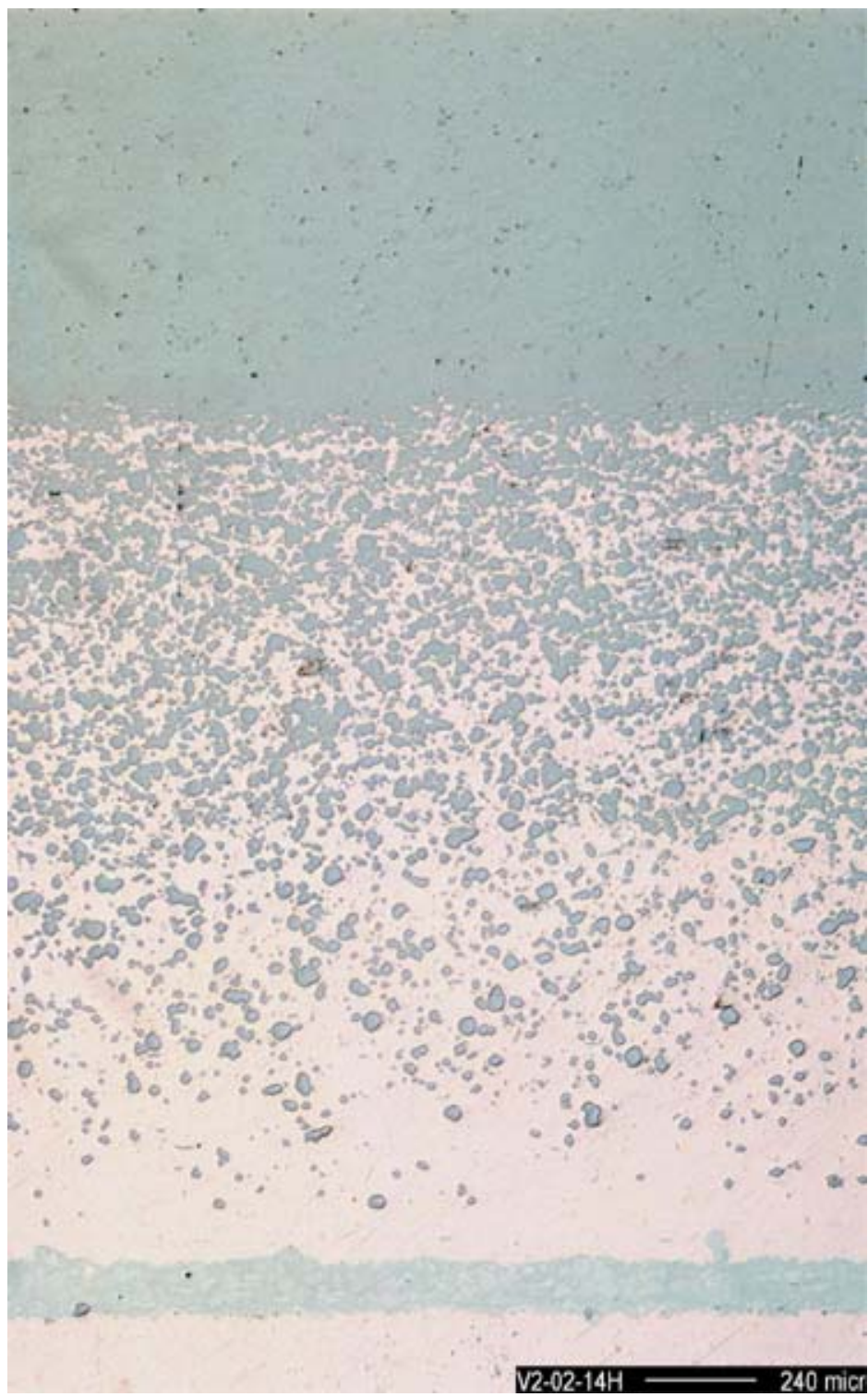

$\sqrt{2-02-14 H}$
Figure 55 - Photomicrograph showing excellent bond achieved between the $\mathrm{CuCrZr}$ heat sink and the VPS deposited material using the bondcoat and the $\mathrm{Cu}$-rich functional gradient joint. 


\subsection{SUMMARY}

Innovative VPS forming techniques were developed that enabled the fabrication of medium scale copper alloy heat sinks armored with thick tungsten deposits, i.e., $3-5 \mathrm{~mm}$. This effort has demonstrate the viability of VPS forming techniques employing functional gradient materials for joining low expansion tungsten armor to high expansion copper alloy heat sinks for use as plasma facing components. Fittings will be attached to the three medium scale mockups and they will be delivered to SNLs for high heat flux testing. Below is a brief summary of the Phase II effort.

- A finite element model was developed and several different gradient architectures were evaluated.

- It was determined a $2 \mathrm{~mm} \mathrm{Cu}$-rich gradient was the optimum gradient architecture.

- The mechanical and physical properties of VPS copper and tungsten were determined to optimize the fabrication techniques.

- The effect of oxygen content in the feedstock powder on the mechanical properties of VPS copper deposits was determined.

- Regarding ultimate tensile and yield strengths, the samples produced with the low oxygen feedstock powder showed more consistent results independent of the sample condition. In contrast, the samples produced with the high oxygen feedstock powder showed noticeable improvements in tensile and yield strength after heat treating.

- Regarding elongation, the samples produced with the low oxygen copper feedstock powder were significantly more ductile than the samples produced with the high oxygen feedstock powder. Samples in the heat treated conditions did show improvements, but were still inferior to the results for the low oxygen feedstock powder.

- Densities of the VPS copper deposits range between $97.6-100 \%$ of theoretical depending on sample condition. The highest densities were achieved for samples that were processed using a hot isostatic pressure (HIP) heat treatment.

- Thermophysical properties testing showed VPS copper has a thermal conductivity of $\sim 87 \%$ of pure copper at room temperature and $\sim 92 \%$ of pure copper at $300^{\circ} \mathrm{C}$.

- Thermophysical properties testing showed VPS tungsten after high temperature heat treating can have a thermal conductivity equivalent to wrought tungsten. However, the thermal conductivity of VPS tungsten in the as-sprayed condition is $\sim 85 \%$ of wrought tungsten at room temperature.

- A VPS procedure was developed to produce $2 \mathrm{~mm} \mathrm{Cu}$-rich gradients for joining tungsten armor to $\mathrm{CuCrZr}$ heat sinks.

- A Cu-Ni bondcoat was employed to enhanced the development of a metallurgical bond between the VPS deposited material and the copper alloy heat sink.

- Dual cooling channel, medium scale mockups were produced with VPS tungsten armor. One mockup was produced with $3 \mathrm{~mm}$ thick tungsten armor and two mockups were produced with $5 \mathrm{~mm}$ thick tungsten armor.

- Residual stresses in the deposited material caused slight bowing of the copper heat sinks after deposition. However, no debonding was observed for any of the three mockups.

- Electric discharge machining techniques were developed for castellation of the armor after spraying. Machining of the tile breaks in the $3 \mathrm{~mm}$ thick tungsten armor was 
sufficient to eliminate the residual fabrication stresses, which resulted in elimination of the bow in the heat sink.

- Although machining of the tile breaks in the samples with $5 \mathrm{~mm}$ thick armor did relieve some residual stresses from fabrication, a slight amount of bow was still present in each mockup.

- Metallographic examination of the interface between the $\mathrm{CuCrZr}$ heat sink and the VPS deposited material showed excellent bonding was achieved.

\subsection{REFERENCES}

1. R. E. Nygren. 1997. A comparison of stresses in armor joints with and without interlayers. Sandia National Laboratories Report SAND97-1495C.

2. R. E. Nygren. 1997. Stress analysis of $\mathrm{W}$ armor with and without interlayers. Sandia National Laboratories Draft of Technical Note.

3. R. E. Nygren. 1997. ABAQUS Input Files. Albuquerque N.M

4. Heat Transfer and Thermal Stress Analysis with ABAQUS. 1997. Hibbitt, Karlsson \& Sorensen, INC.

5. ABAQUS/Standard User's Manual Version 5.6. 1996. Hibbitt, Karlsson \& Sorensen, INC.

6. P.J. Karditsas, M.J. Baptiste. Thermal and Structural Properties of Fusion related Materials. Fusion Energy Research Program UCSD, San Diego CA.

7. Plasma Processes. Thermal Spray Coatings and Design Services. 1997. Age hardened Elbrodur G properties table.

8. R. L. Williamson, B. H. Rabin, and J. T. Drake. 1993. Finite element analysis of thermal residual stresses at graded ceramic-metal interfaces. Part I. Model description and geometrical effects. J. Appl. Phys. 74 : 1310-1320.

9. J. T. Drake, R. L. Williamson, and B. H. Rabin. 1993. Finite element analysis of thermal residual stresses at graded ceramic-metal interfaces. Part II. Interface optimization for residual stress reduction. J. Appl. Phys. 74 : 1321-1326.

10. H.A. Bruck and B.H. Rabin. 1997. Evaluating microstructural and damage effects in rule-of-mixtures predictions of the mechanical properties of $\mathrm{Ni}_{-} \mathrm{Al}_{2} \mathrm{O}_{3}$ composites for use in modeling functionally graded materials. Submitted to J. Mater. Sci.

11. S. Suresh and A. Mortensen. 1997. Functionally graded metals and metal-ceramic composites: Part 2 Thermomechanical behavior. Int. Mater. Rev. 42 : 85-116. 
12. W. D. Kingery, H. K. Bowen and D. R. Uhlmann. 1976. Introduction to Ceramics. New York. John Wiley \& Sons.

13. K.K. Chawla. 1993. Ceramic Matrix Composites. New York. Chapman \& Hall.

14. R.D. Torres, G.G.W. Mustoe, I.E. Reimanis and J.J. Moore. 1996. Evaluation of residual stresses developed in a functionally graded material using the finite element technique. Processing and fabrication of advanced materials IV. 431-438.

15. Dieter. 1976. Mechanical Metallurgy. New York. McGraw-Hill.

16. M. Case and Klod Kokini. 1993. Thermally induced initiation of interface edge cracks in multilayer ceramic thermal barrier coatings. Ceramic coatings ASME 44 : 149-162.

17. Y.-D. Lee and F. Erdogan. 1994/1995. Residual/thermal stresses in FGM and laminated thermal barrier coatings. International Journal of Fracture 69 : 145-165.

18. J. H. Lau. 1993. Thermal stress and strain in microelectronics packaging. New York: Van Nostrand Reinnhold.

19. C. H. Hsueh and A.G. Evans. 1985. Residual Stresses in Metal/Ceramic Bonded Strips. J. Am. Ceram. Soc. 68 : 241-248. 
Appendix A: Thermal Residual Stresses and Performance in Cu-W Graded Layers. 

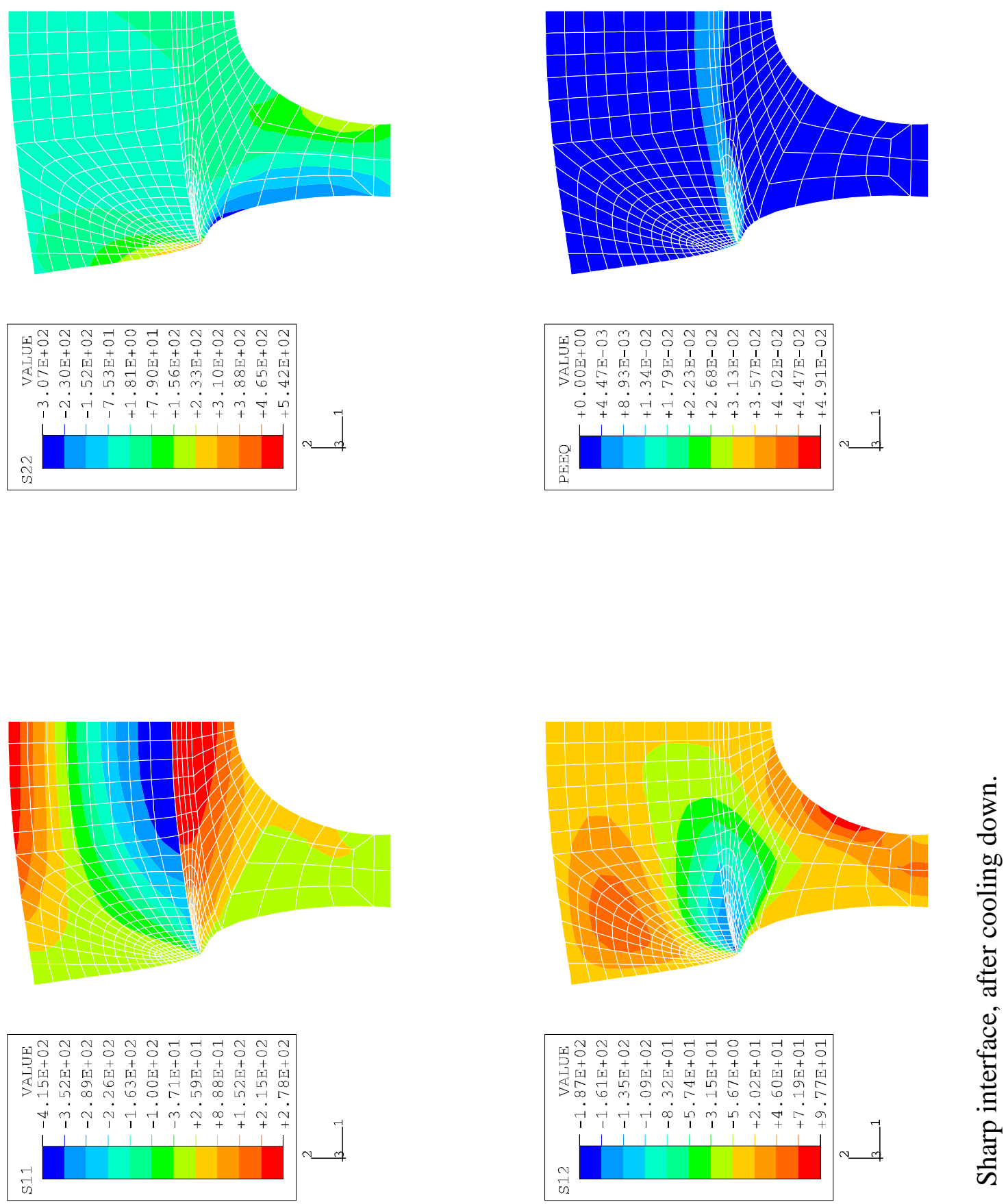

نَّ 

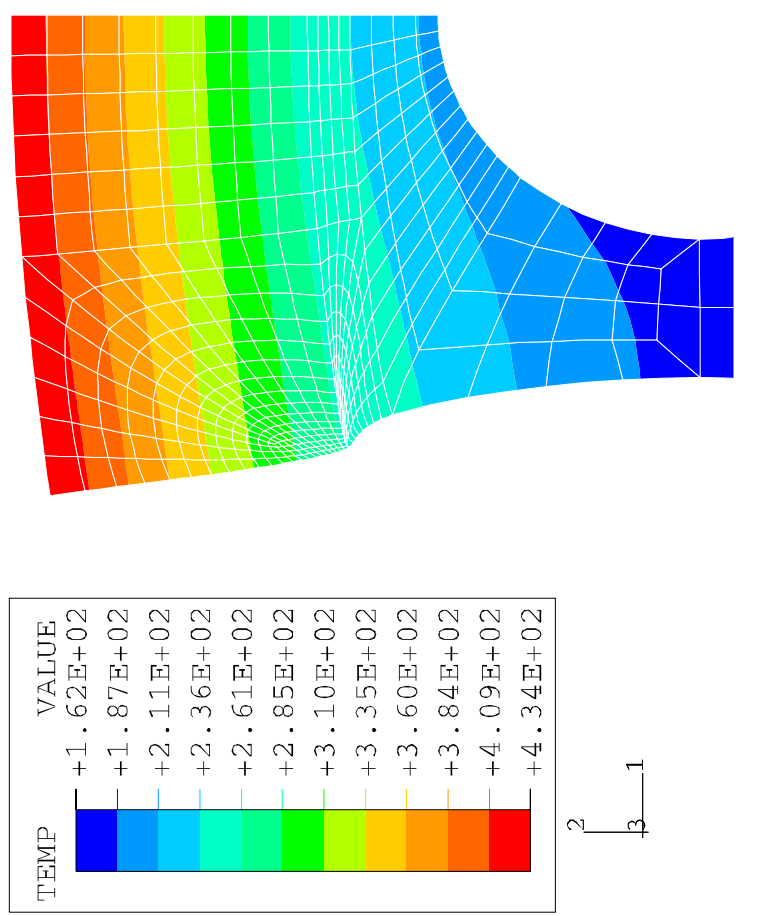

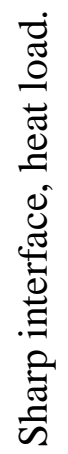



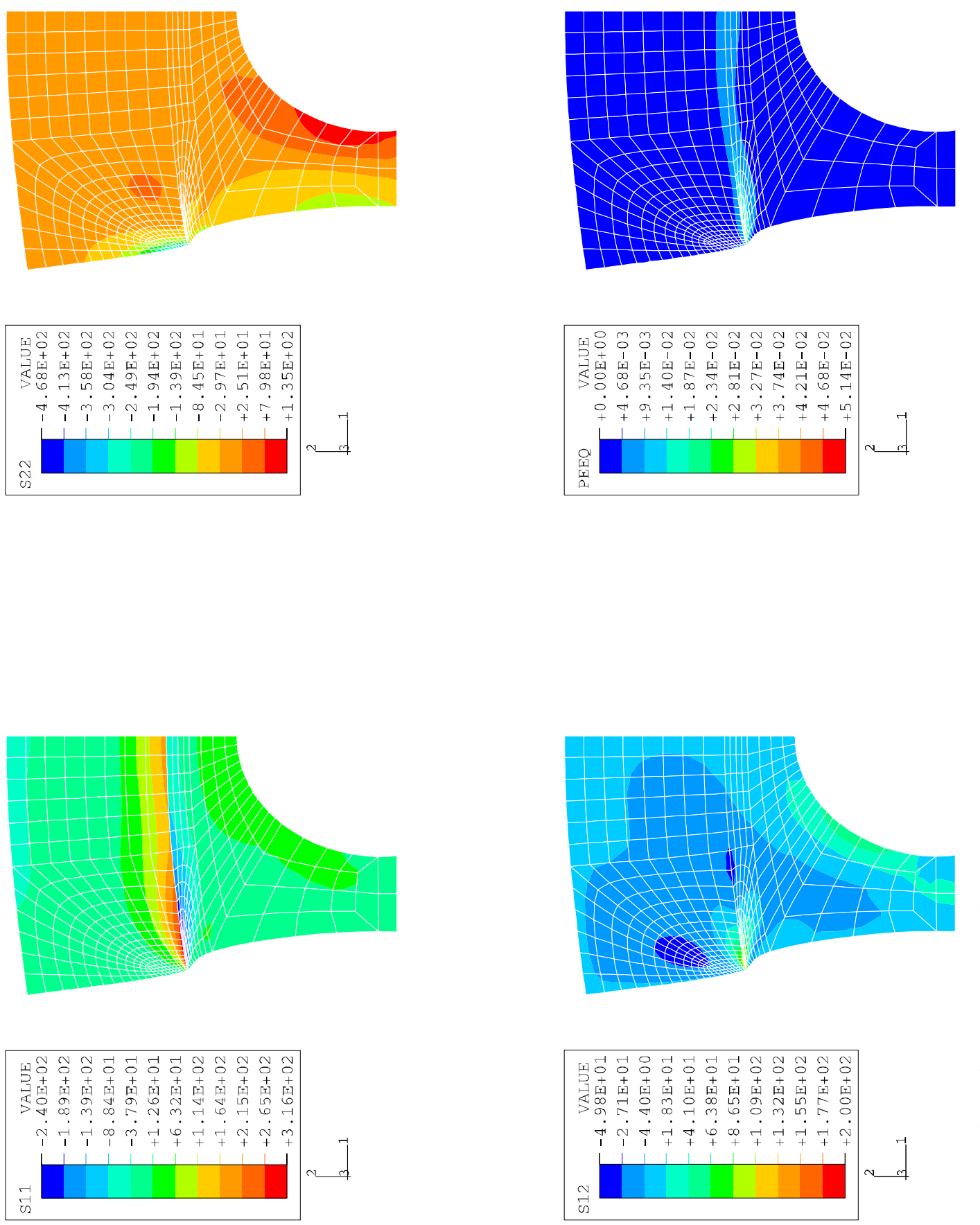

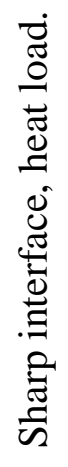



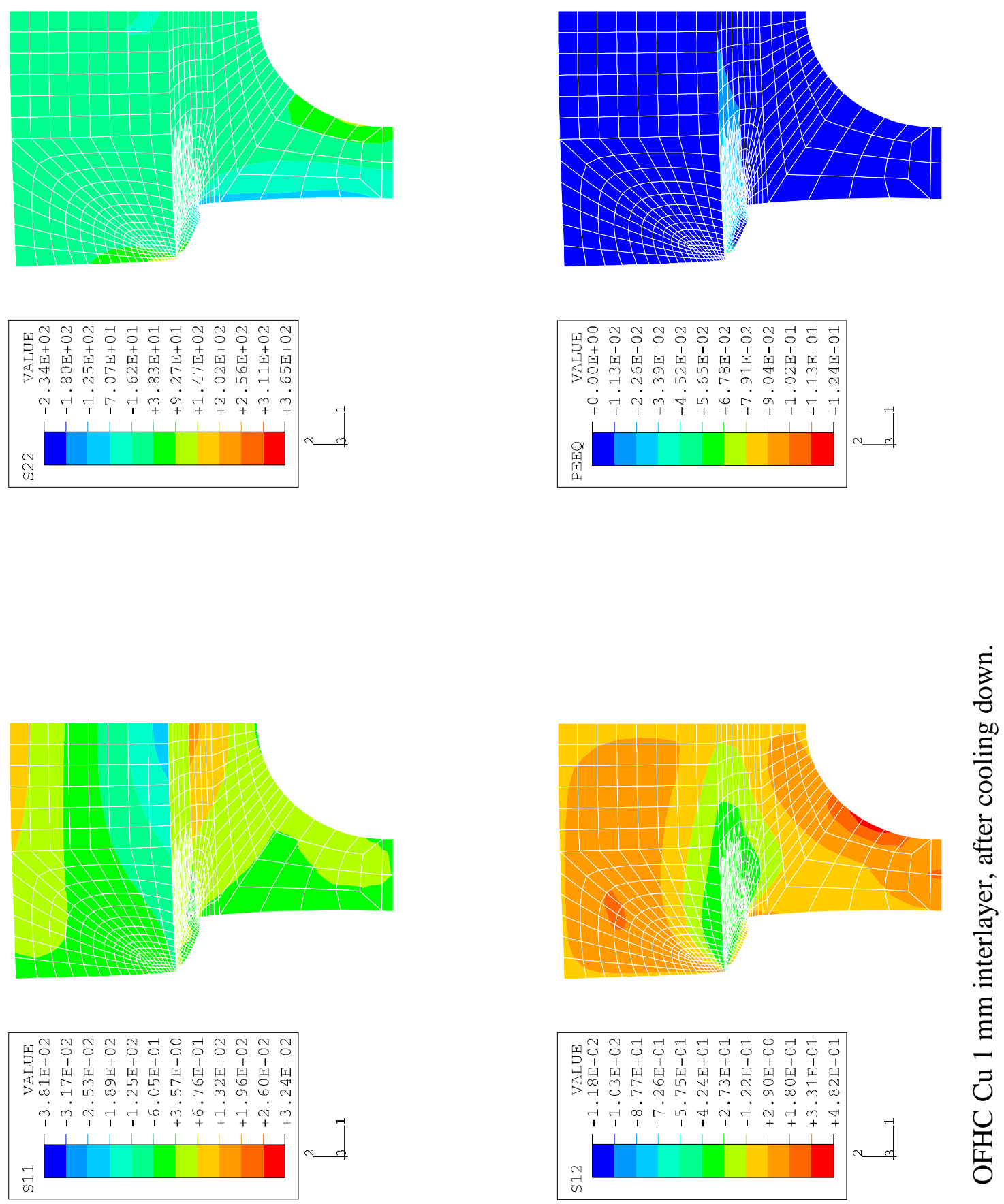

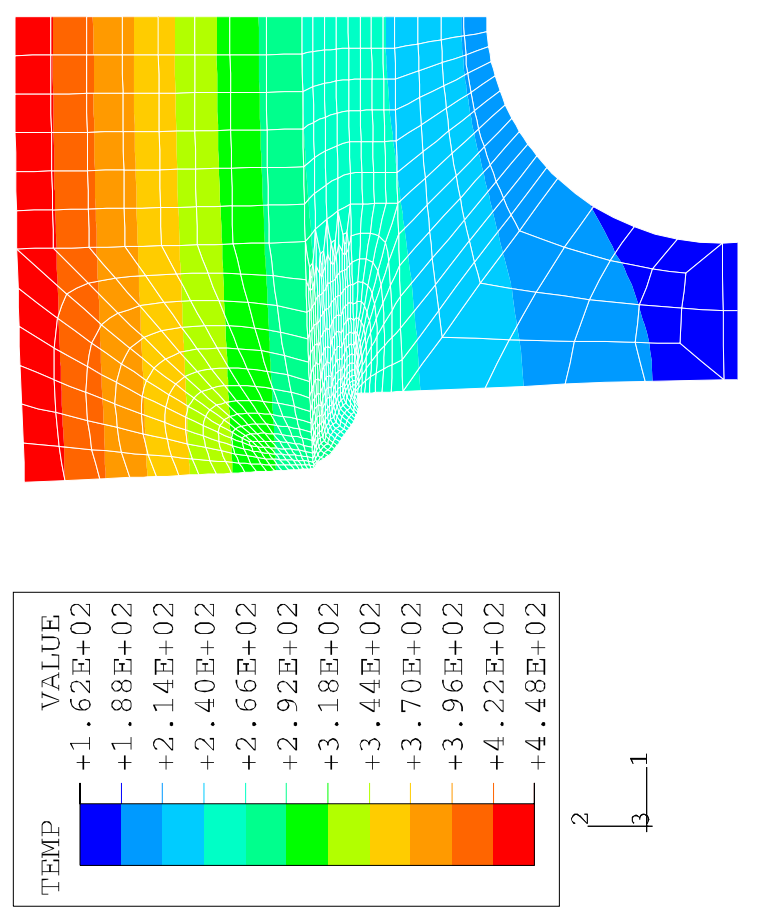

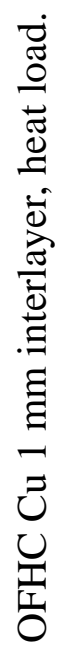



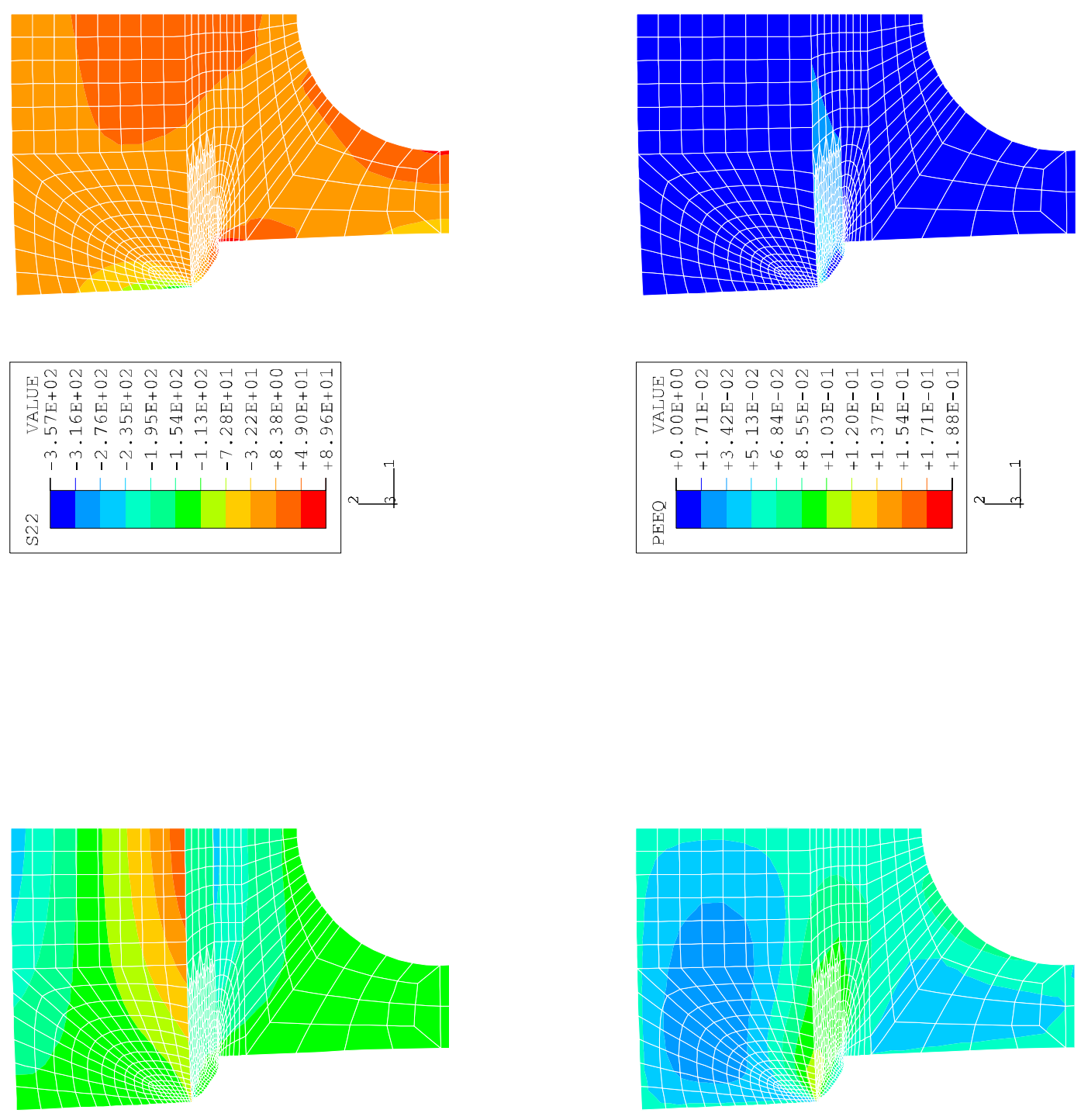

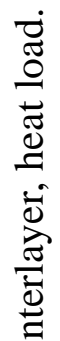
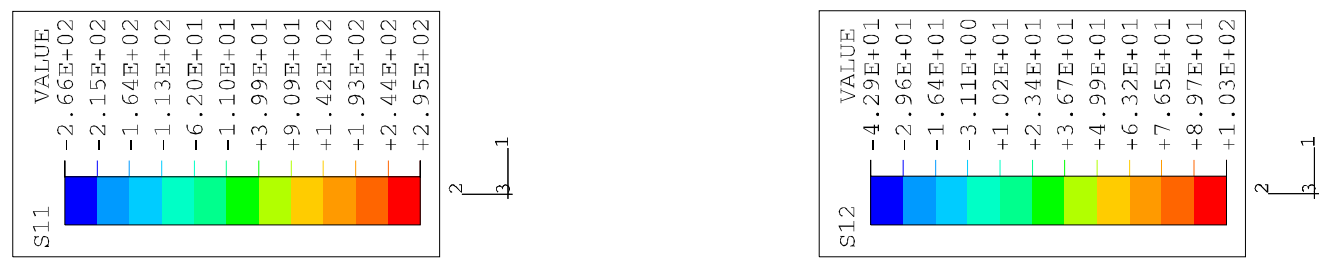

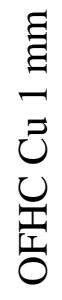



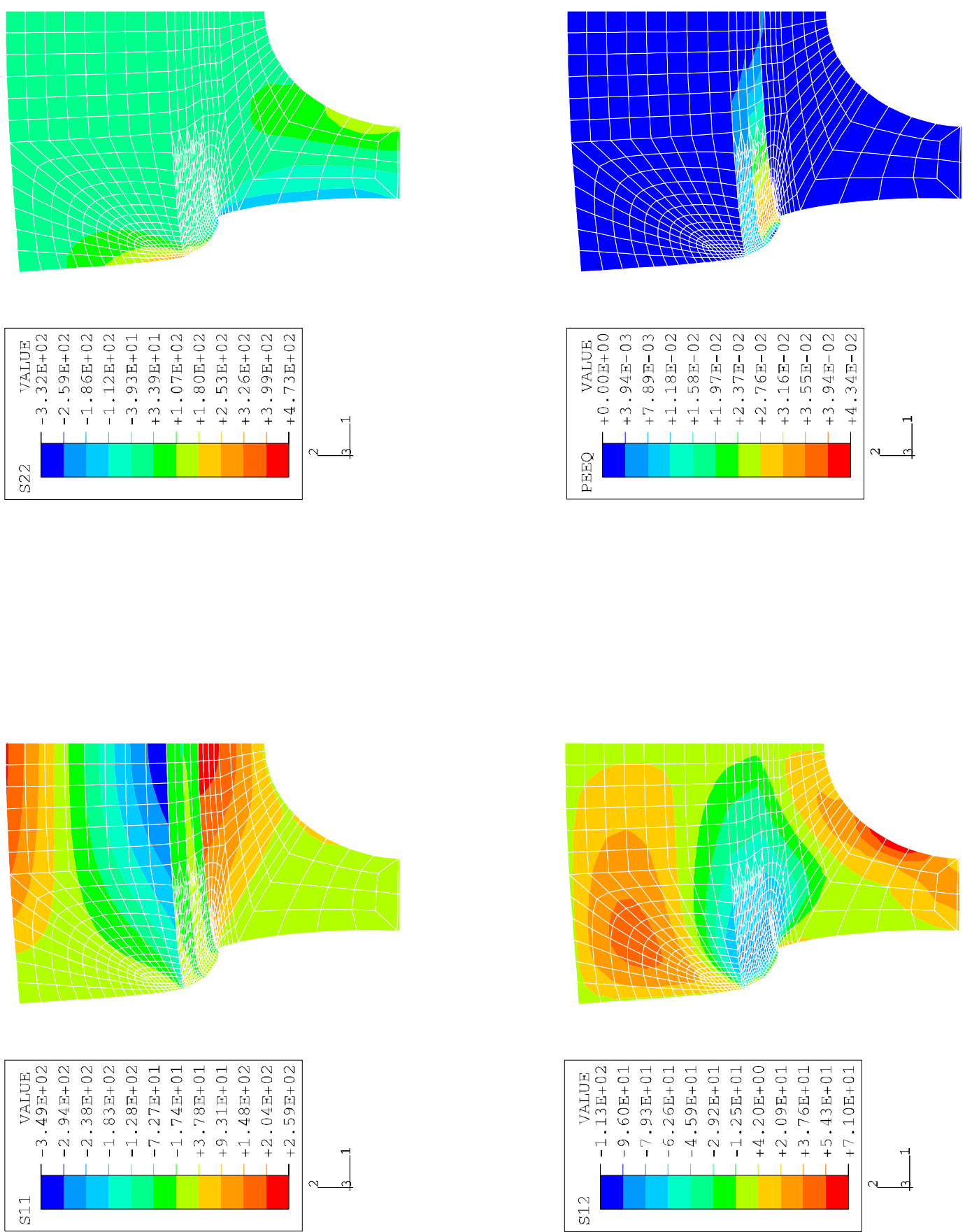

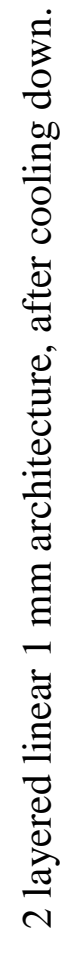



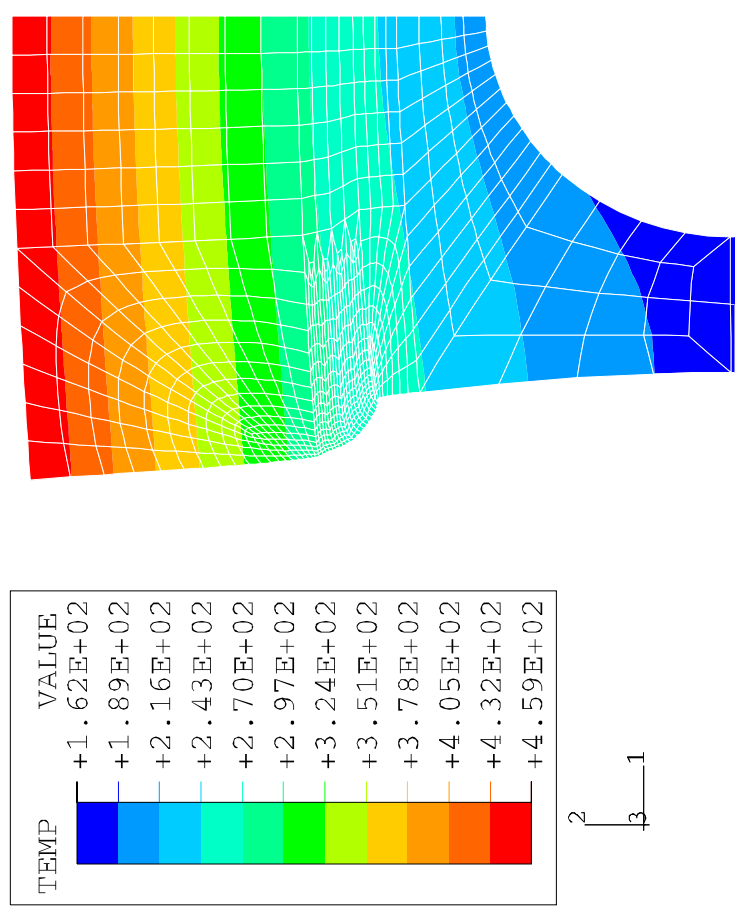

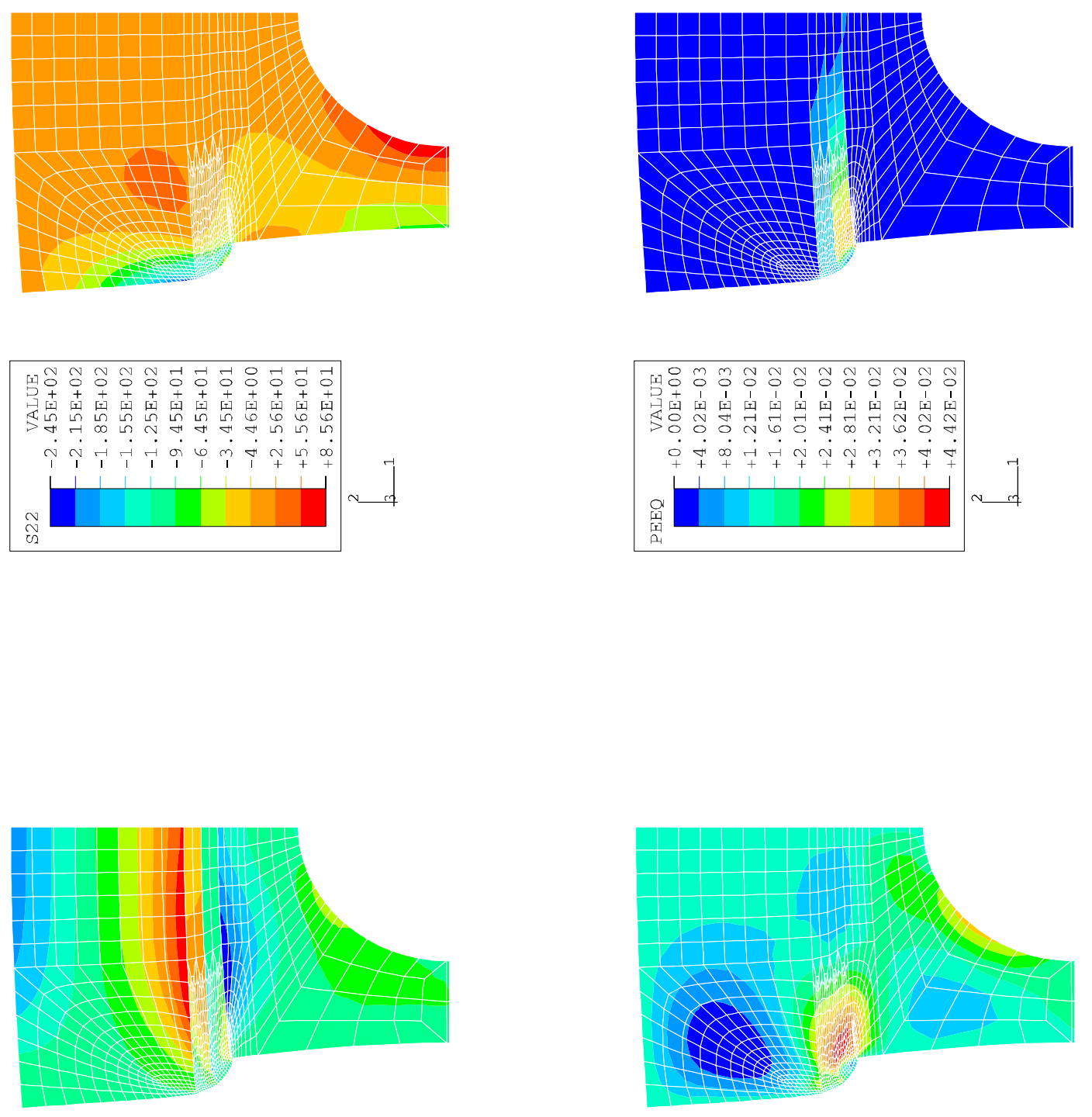

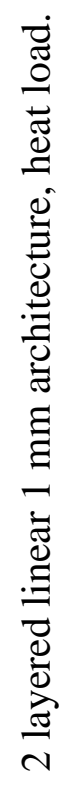
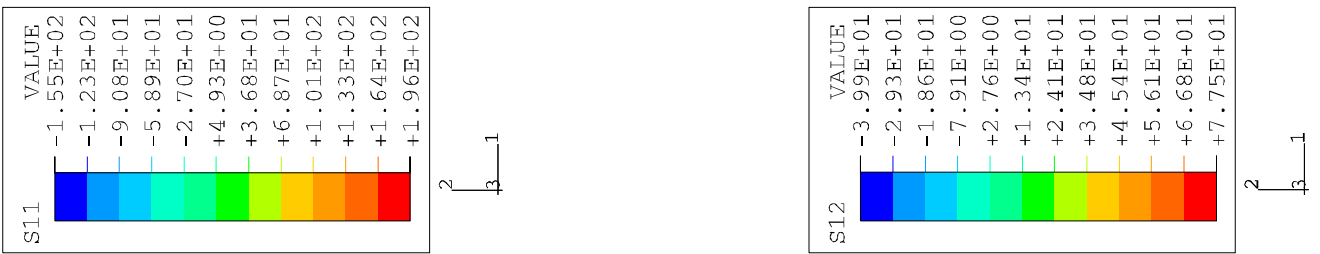

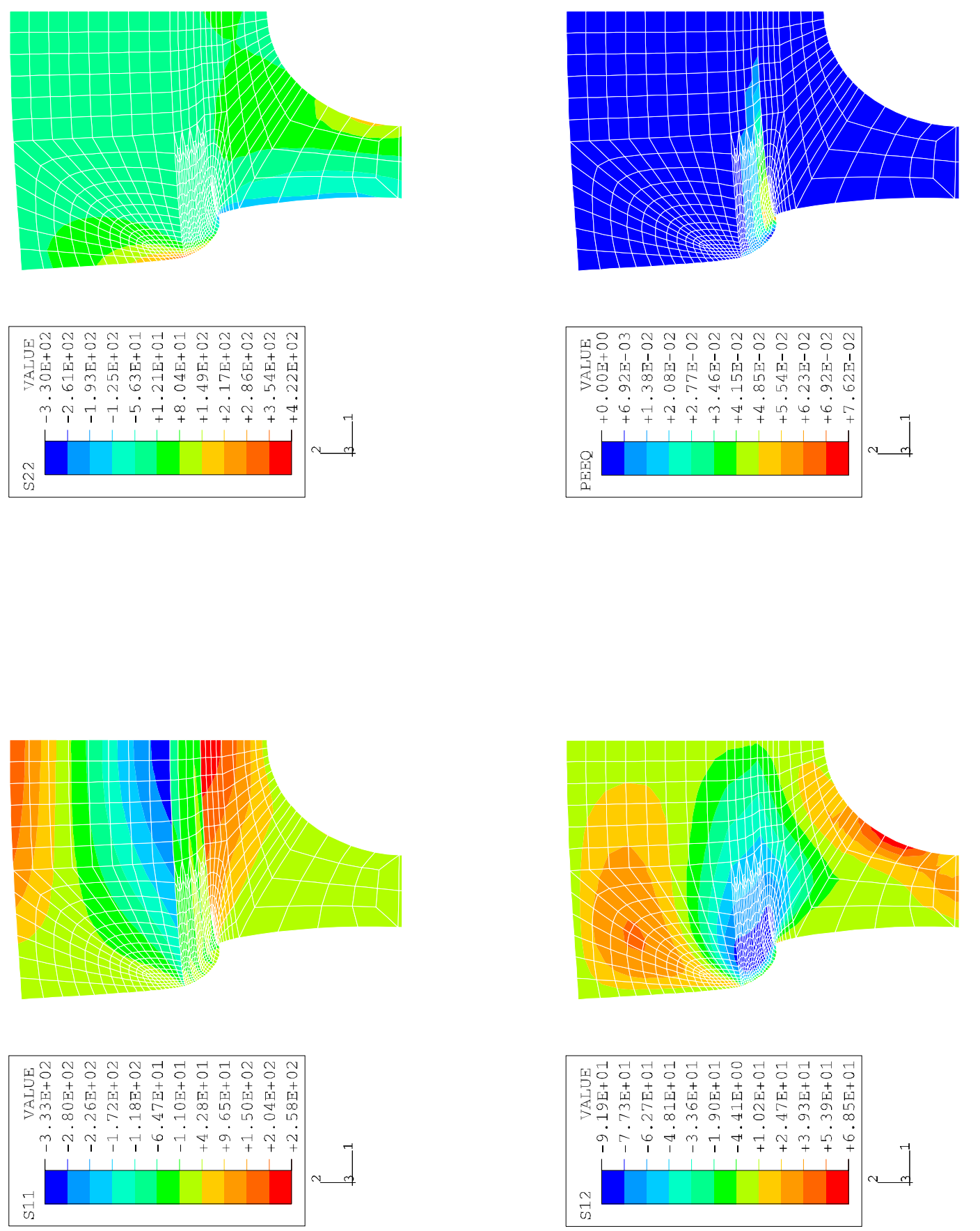

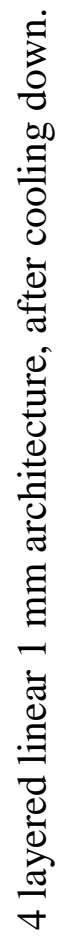



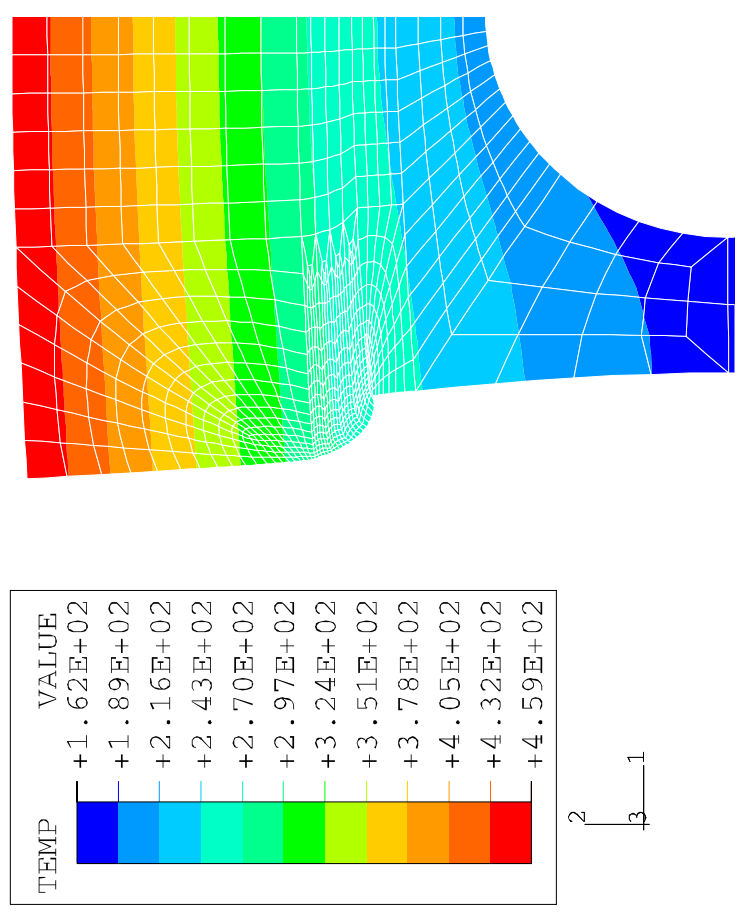

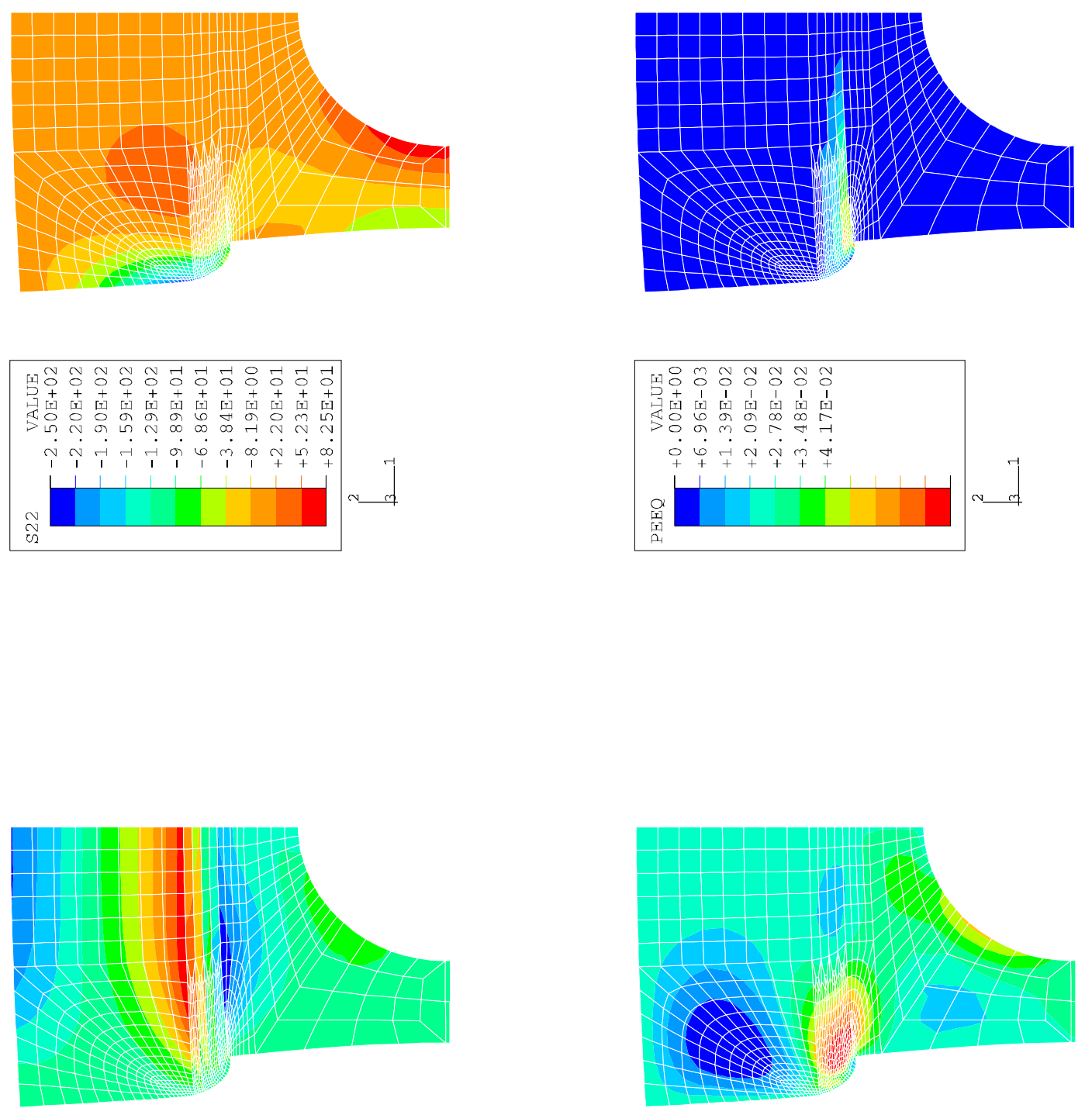

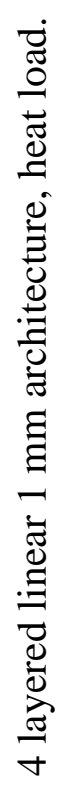
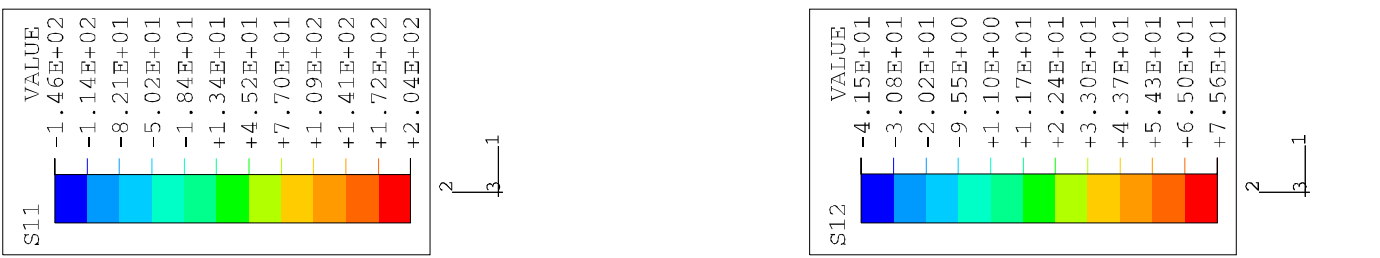

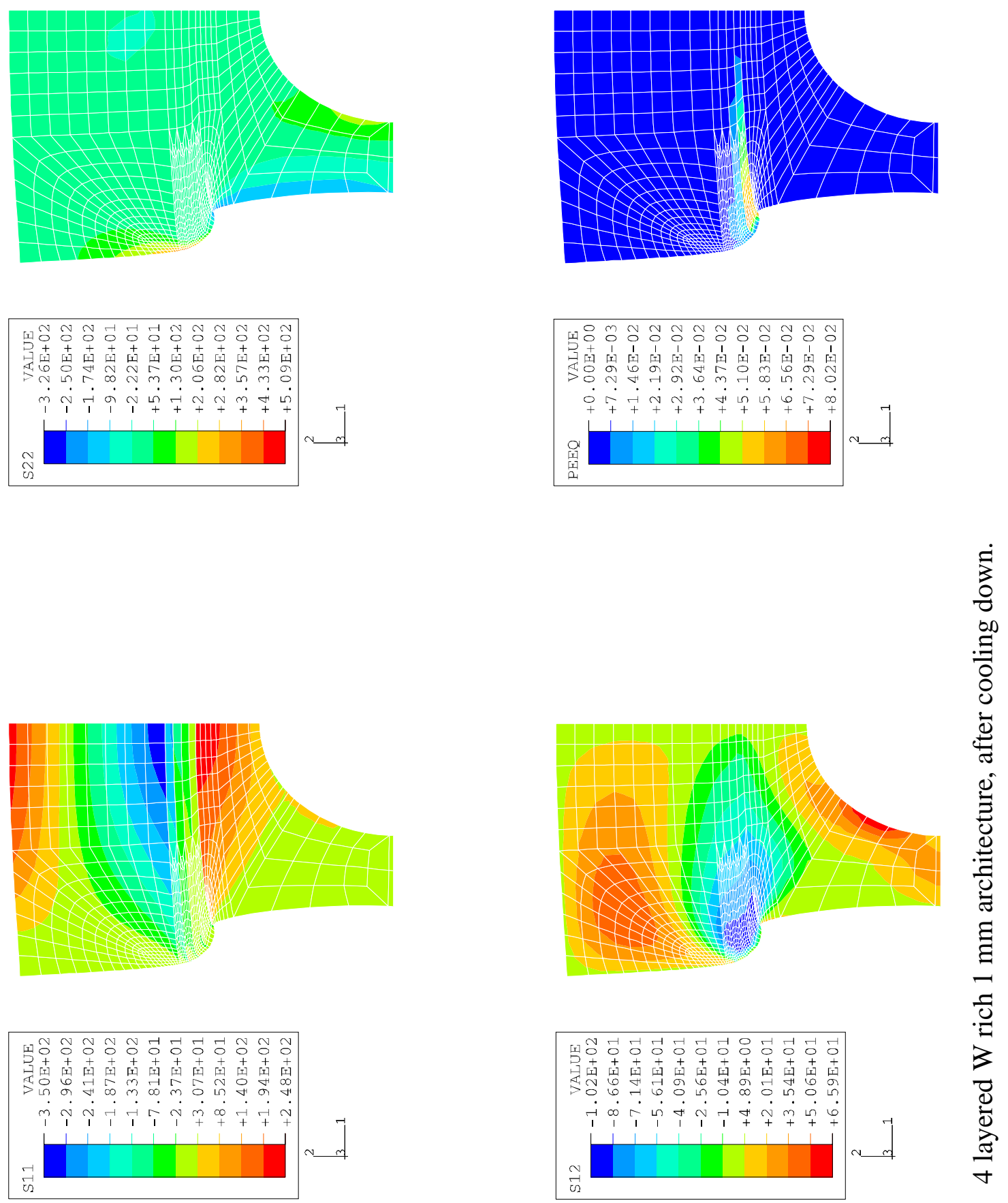

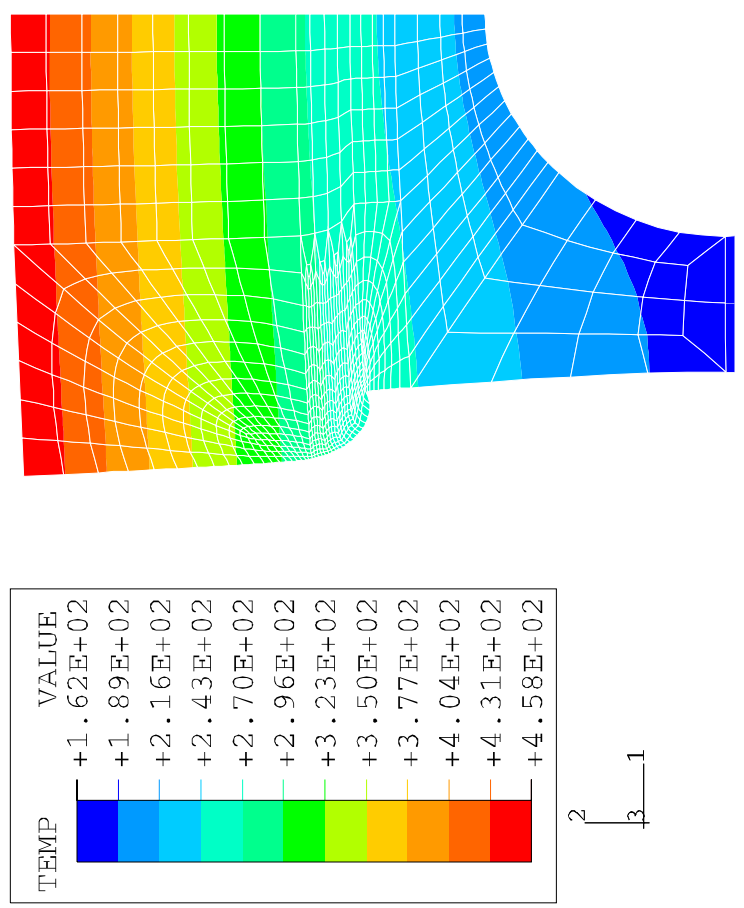

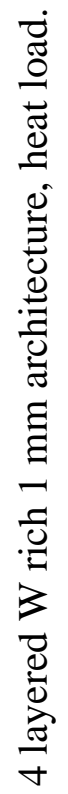



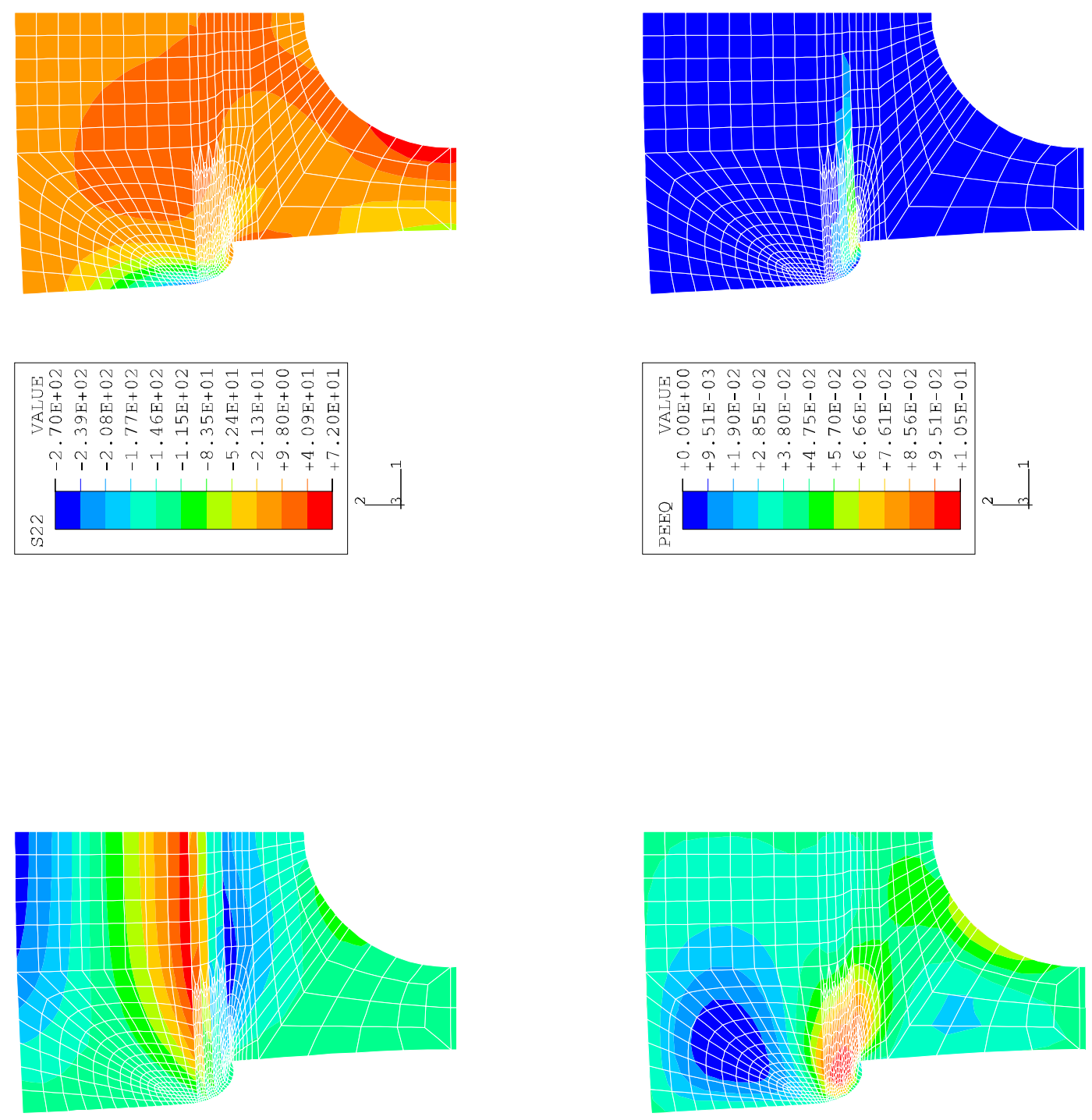

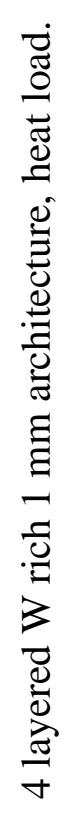
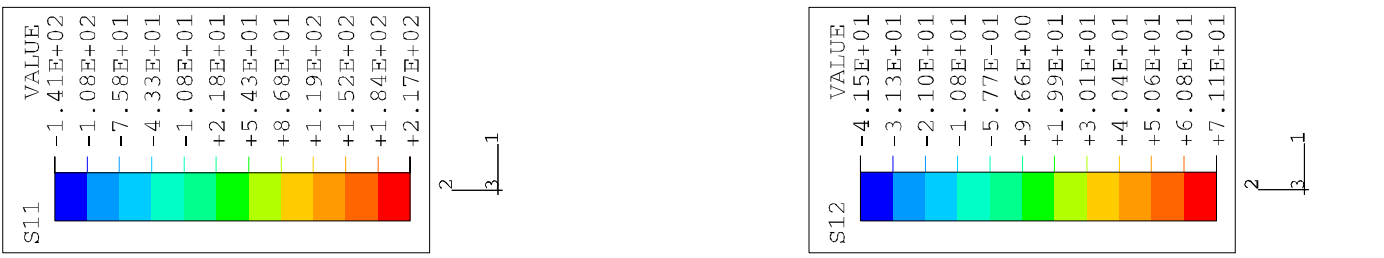

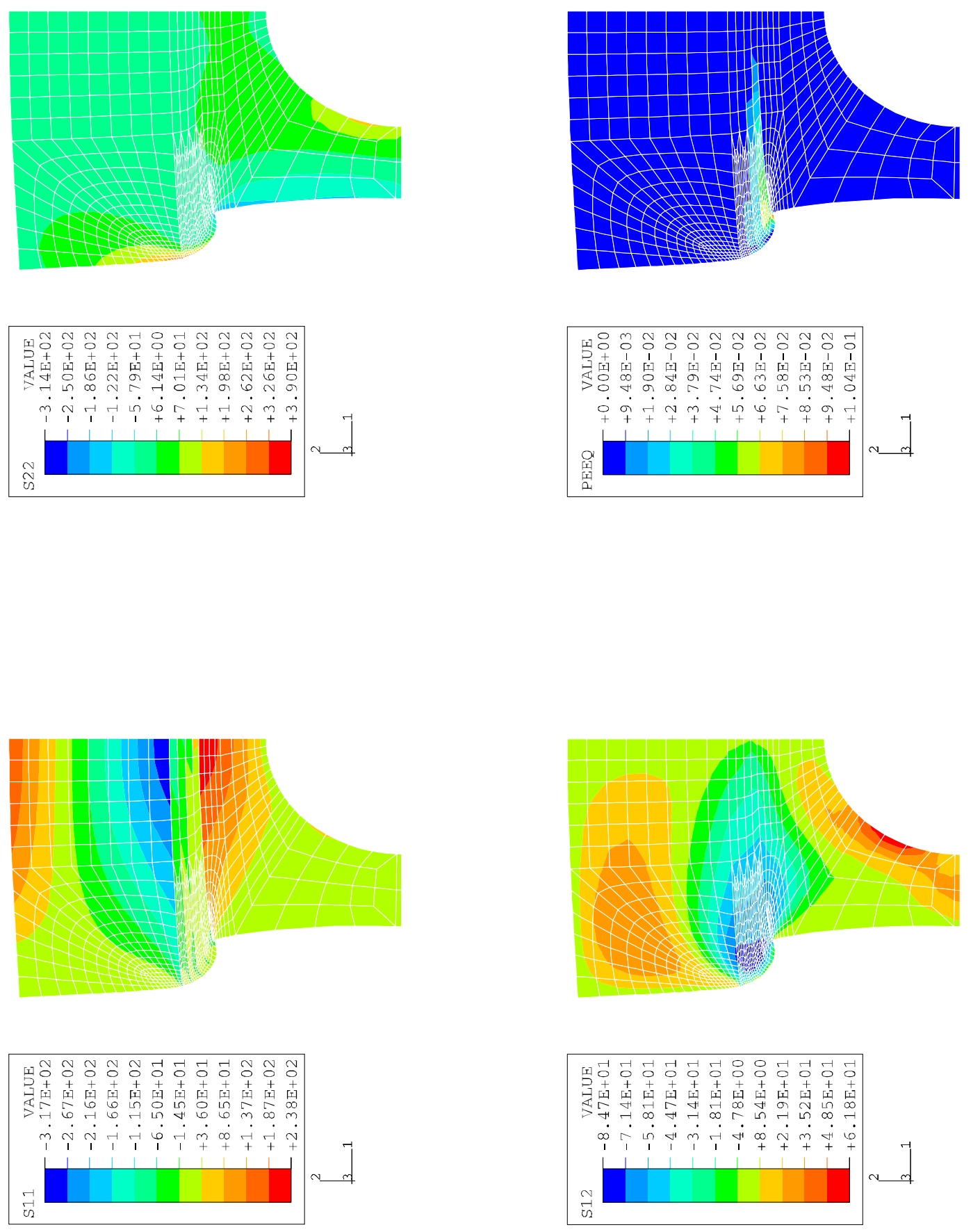

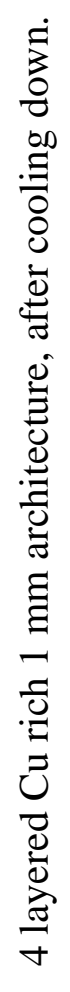



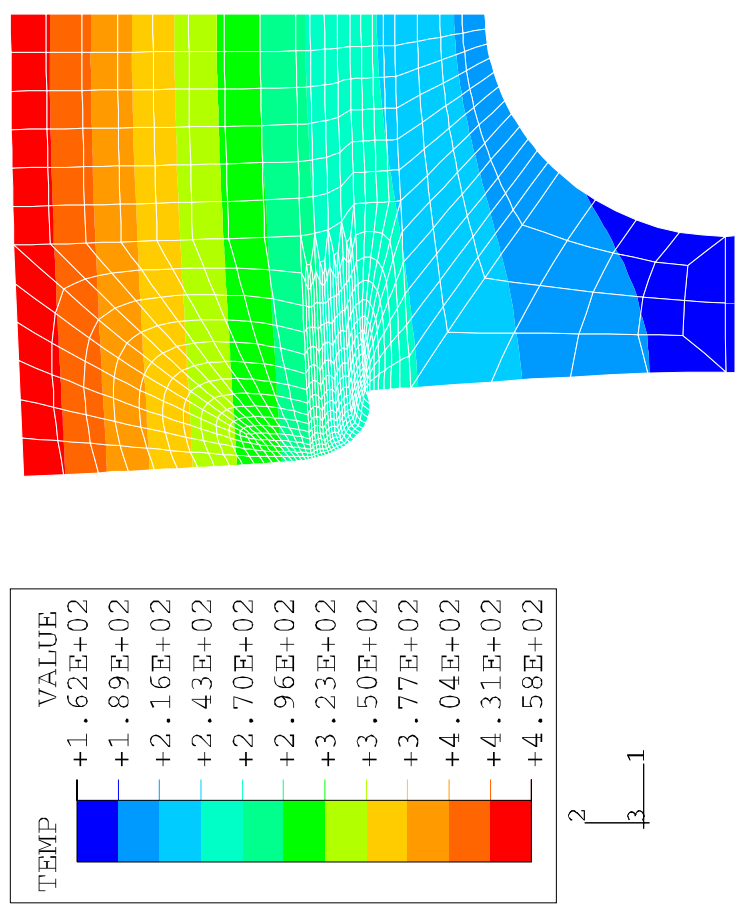

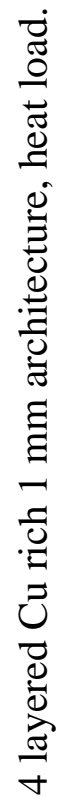



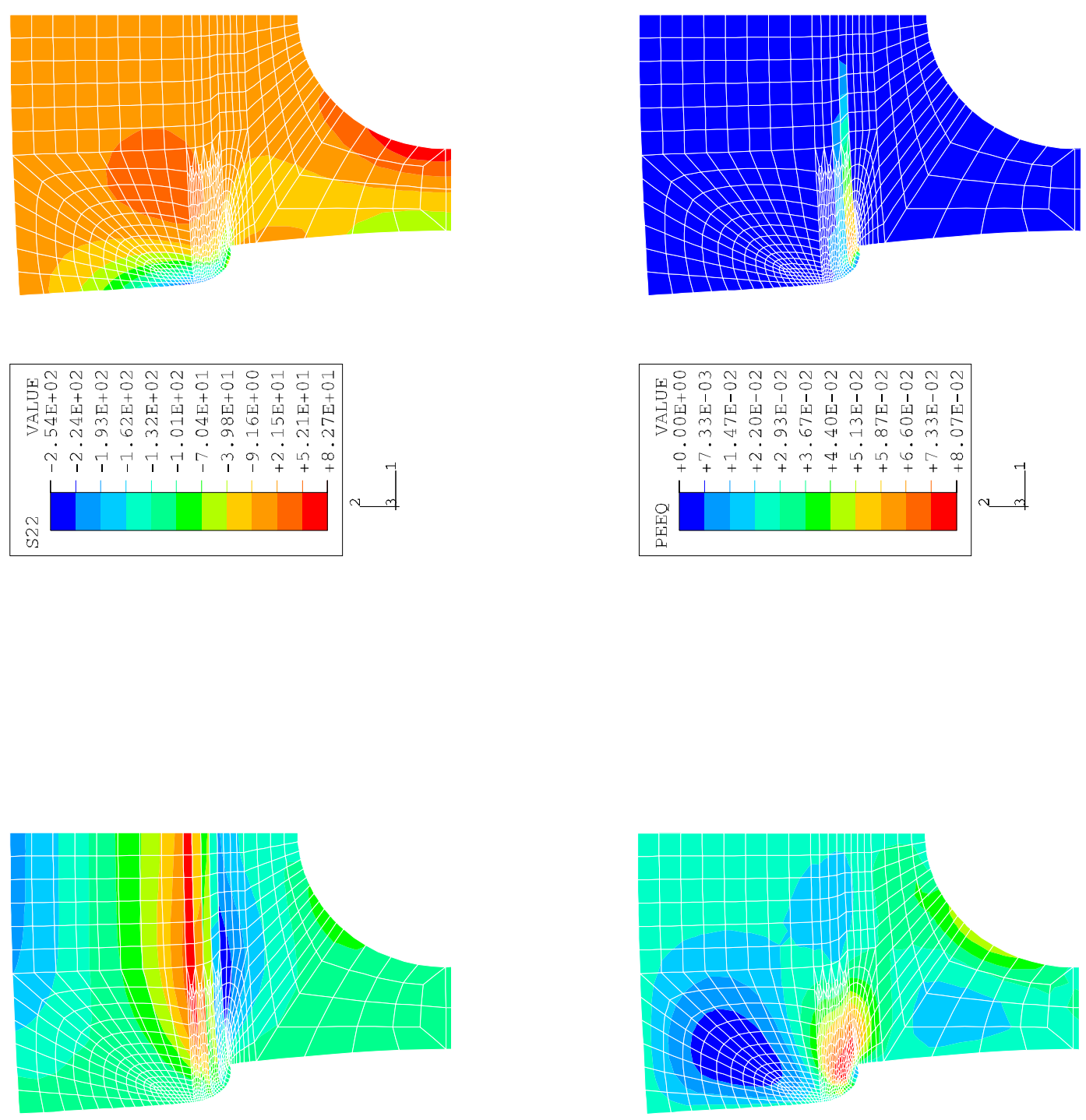

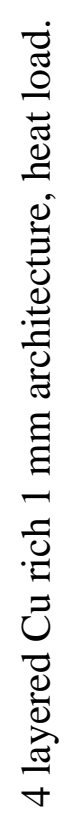
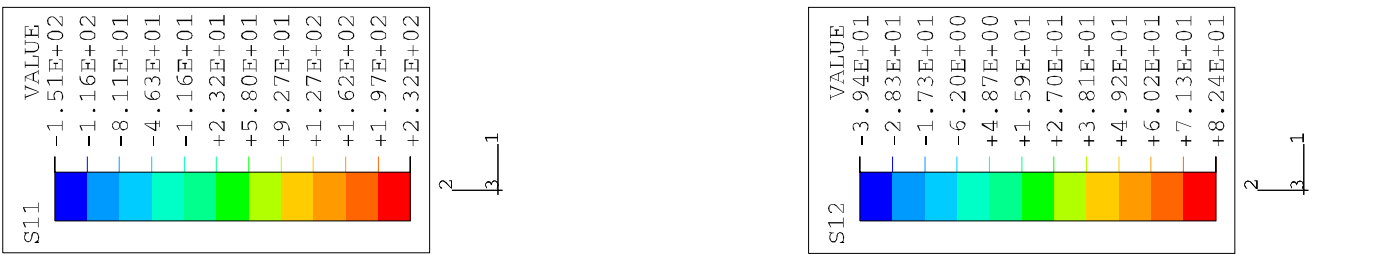

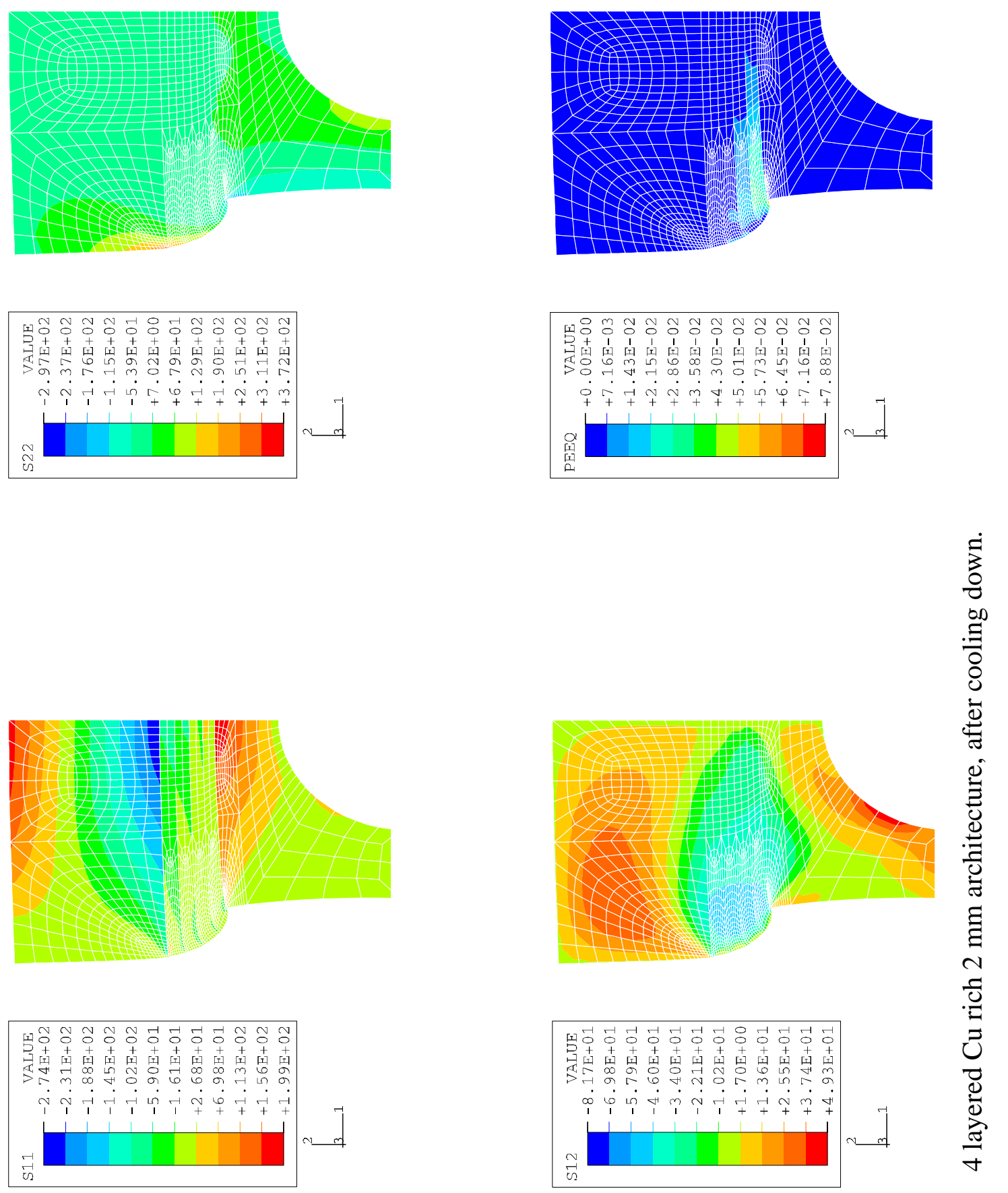

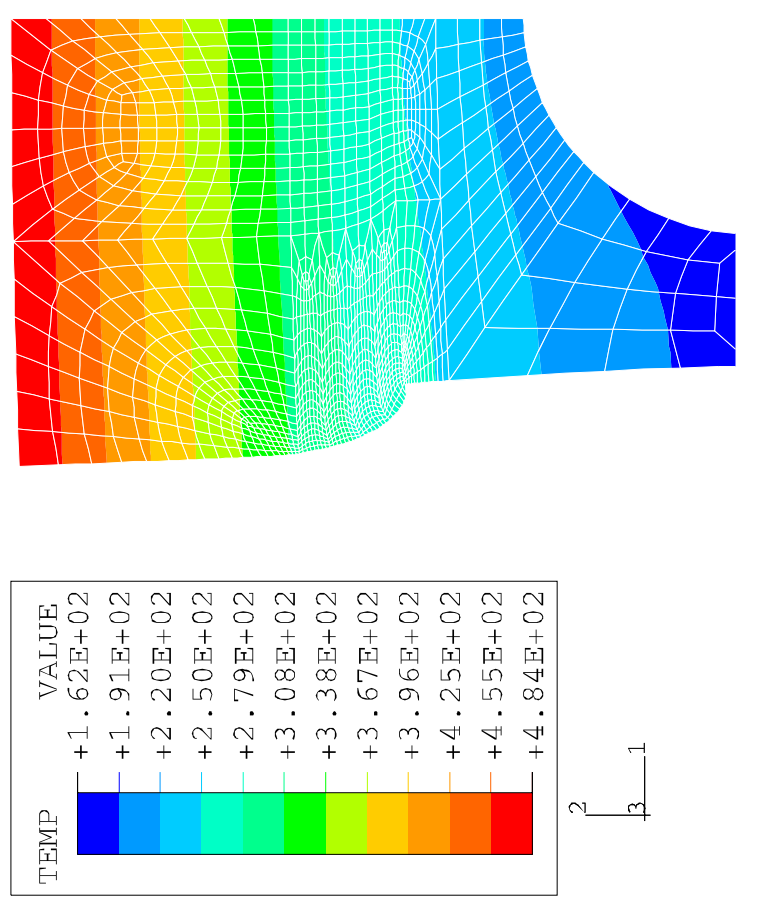

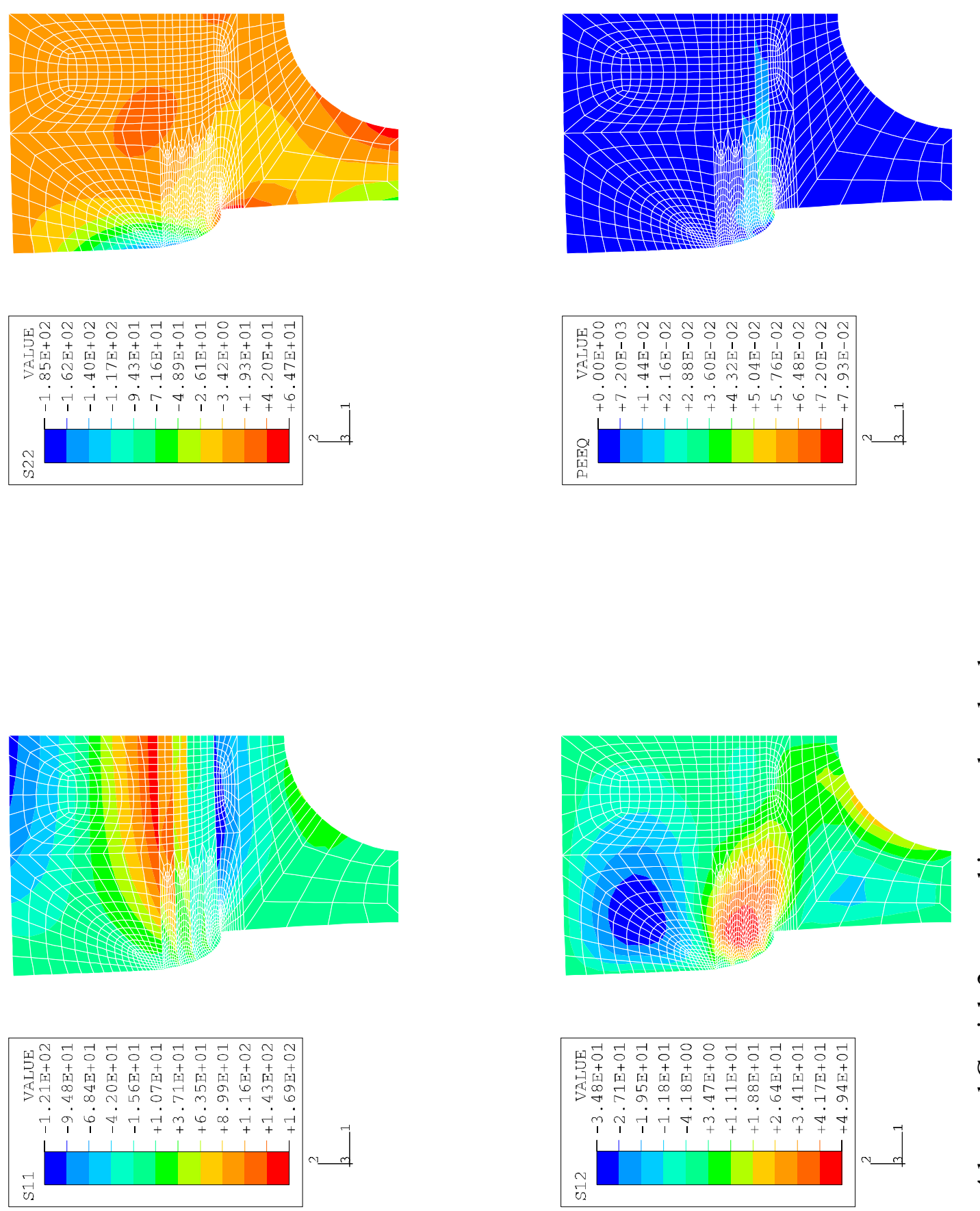

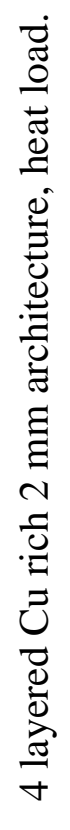



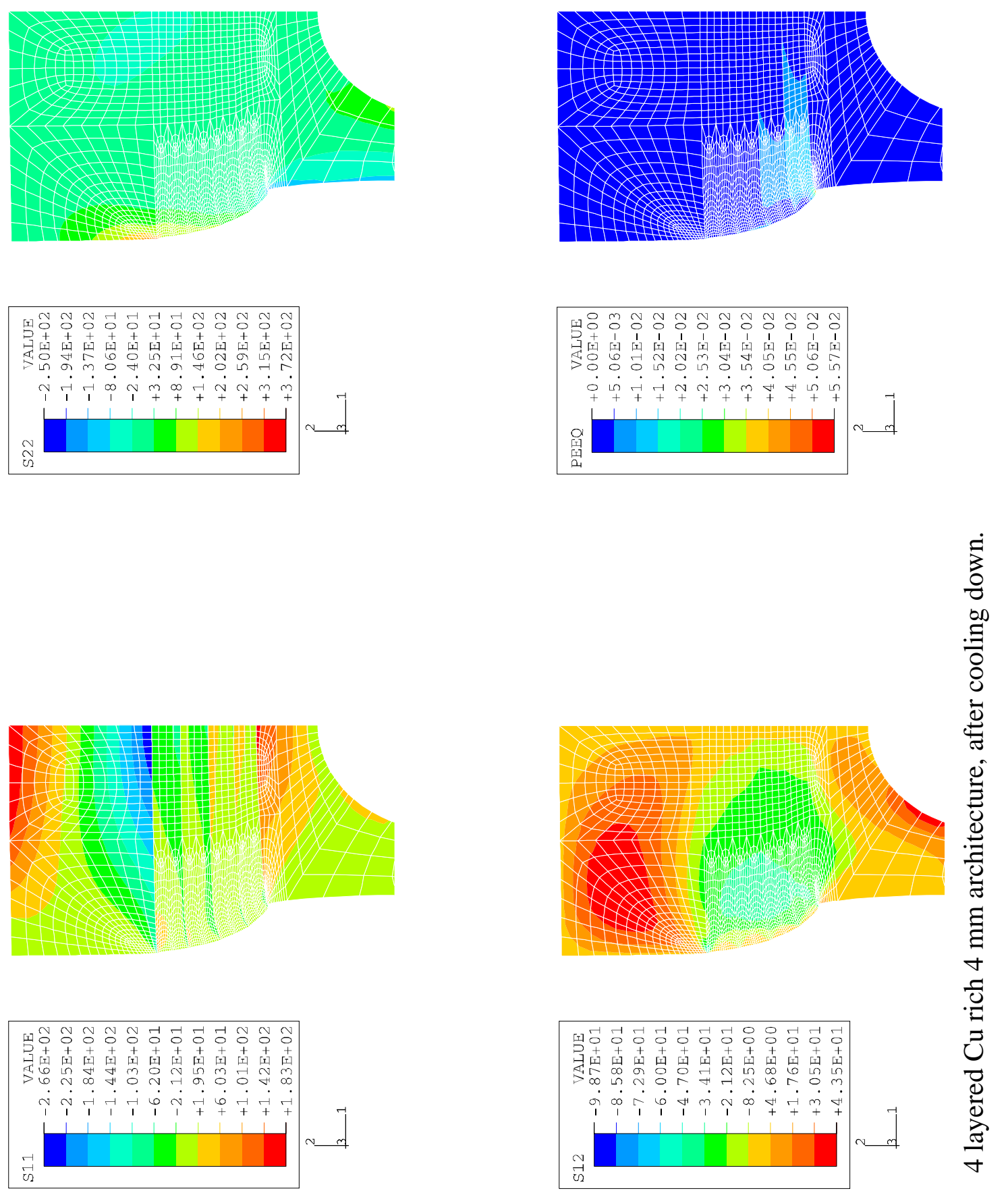

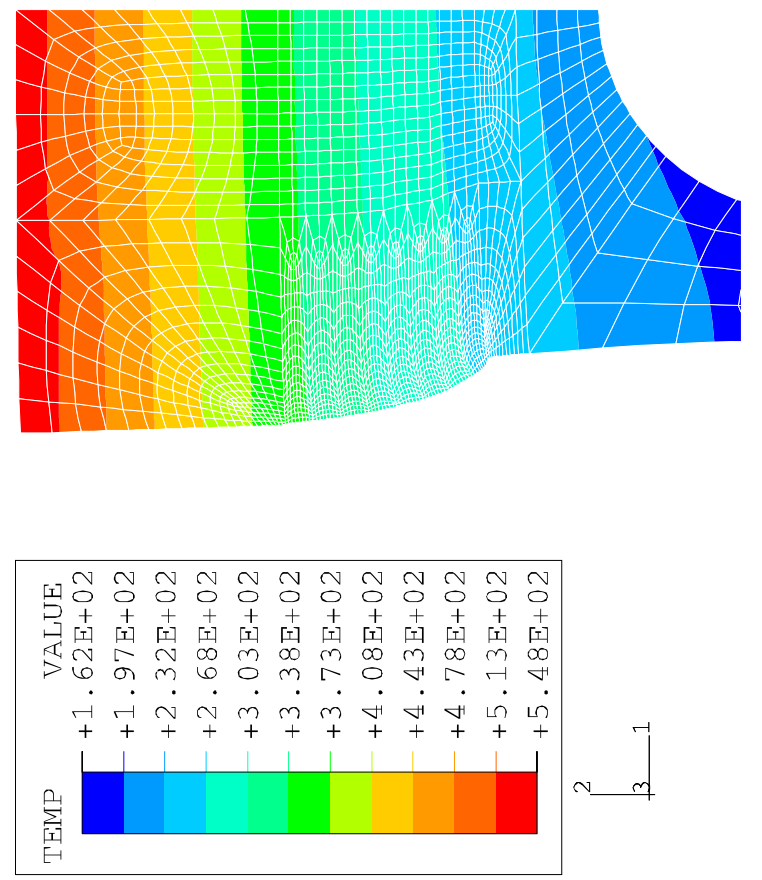

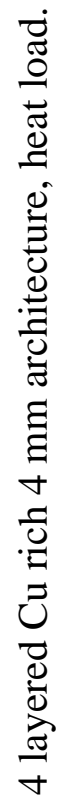



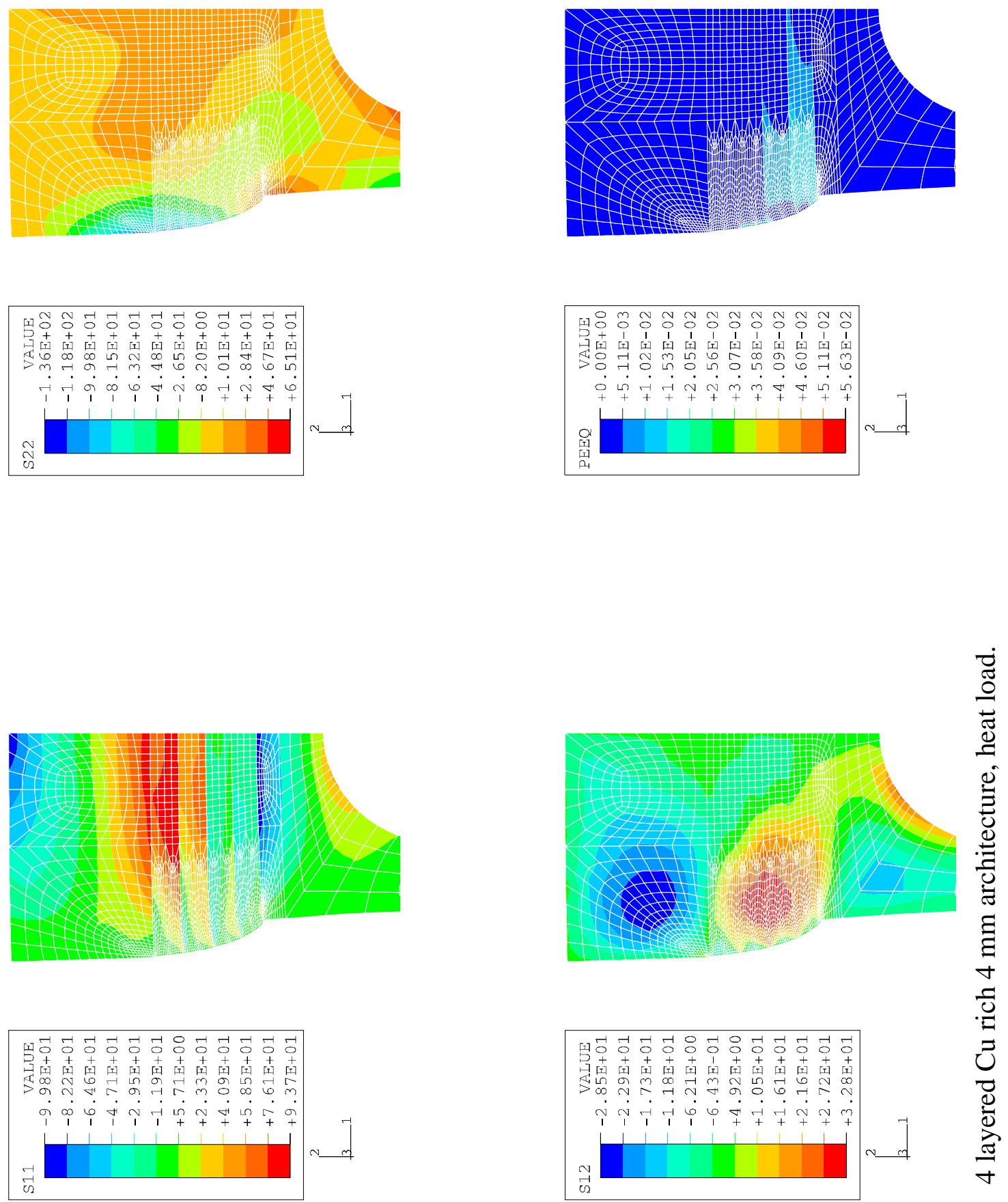

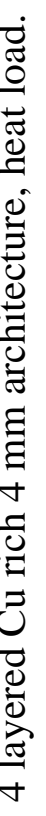




\section{Appendix B - Medium Scale Mockup Gradient Procedure}

\section{2-0531-1 Revision I}

W-Cu Gradient

Nozzle: V129 (82 cathode)

Sample Type:_Cu Bar

Standoff: 7"

Copper Powder:_CEAC -325

Tungsten Powder_Alldyne -325

Monel (Cu (CEAC)-60\%Ni (Crucible))

Part Program:
Start Thickness:

Run \#: V2-02-14I

No. of Passes 40 gradient

Run Time

Lot \#__ W

Lot \# $\mathrm{Cu}$

Lot \# $\mathrm{Ni}$

Cooling: argon to sting and underside of mockup

Powder injection orientation parallel to mockup for copper and tungsten tied line

Powder injection orientation parallel to mockup for monel

\section{Ports: same}

$1 \quad$ Sample preparation

Acetone wipe

Grit blast: 1' standoff, 80-90 psi, 54 grit SiC

$2 \quad$ Drain all powder feeder lines

Set $\mathrm{W}$ feeder to 0 RPM prior to start

Set $\mathrm{Cu}$ feeder to 0 RPM,

Set Monel powder feeder to 2 RPM,

Open copper feed line valve

Evacuate/backfill feeders to 0-2psi

Turn W feeder On/Off switch to On at the feeder

3 Turn argon gas on to sting.

Turn cooling tower fan on

4. Turn torch water on.

Turn torch on:

Argon $\quad 90 \mathrm{psi} \quad 53$ orifice (orifice control \#1)

Hydrogen $90 \mathrm{psi} \quad .009$ orifice (orifice control\#4)

$\mathrm{Set} \mathrm{Cu}$ powder feeder gas flow to $60 \mathrm{psi}$ ( 80 orifice) to bleed out $\mathrm{Cu}$ from line,

Set $\mathrm{W}$ powder feeder gas flow to 6 scfh on argon supply,

Set Monel powder feeder gas flow to $61 /$ minute argon bottle flow $=10 \mathrm{scfh}$ to middle of ball.

Amps 1300

Volts

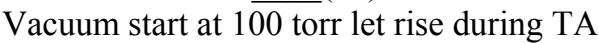

Set $\mathrm{Cu}$ powder feeder gas flow to $30 \mathrm{psi}$,

5 Start preheat traverse Preheat to $1100-1200$ F

6 Transfer arc clean after 6 passes:

Amps

Volts

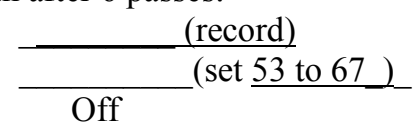

7. At 120 Torr, turn Monel feeder on, 900 amps, primary gas 90 psi Ar, secondary gas 90 psi H2. After one pass of Monel, turn TA off. Turn Monel off after second pass. 
8. Turn Cu Feeder On/Off Switch to ON at the feeder

9. $\quad$ Every pass increase W Feeder \#7 to $2.5 \mathrm{RPM}$ and decrease $\mathrm{Cu}$ feeder to zero.

GRADUALLY INCREASE ARC CURRENT AND HYDROGEN

10. During tungsten deposition (pass 39-n), turn argon cooling on to mockup ( 1/2 turn).

\begin{tabular}{|c|c|c|c|c|c|c|c|c|c|c|c|c|}
\hline \multirow[t]{2}{*}{ Pass \# } & \multicolumn{4}{|c|}{ Gun Parameters } & \multicolumn{3}{|c|}{ Carrier Gass } & \multicolumn{3}{|c|}{ Feed Rate (rpm) } & \multirow[t]{2}{*}{ TA } & \multirow[t]{2}{*}{ Volts } \\
\hline & $\begin{array}{l}\text { Ar } \\
\text { psi }\end{array}$ & $\begin{array}{l}\mathrm{H} 2 \\
\text { psi }\end{array}$ & $\begin{array}{l}\text { Amp } \\
\mathrm{s}\end{array}$ & $\begin{array}{l}\text { Vacuu } \\
\mathrm{m}\end{array}$ & $\begin{array}{l}\text { Monel } \\
\mathrm{L} / \mathrm{min}\end{array}$ & $\begin{array}{l}\mathrm{Cu} \\
\text { psi }\end{array}$ & $\begin{array}{l}\text { W } \\
\text { scfh }\end{array}$ & Monel & $\mathrm{Cu}$ & $\mathrm{W}$ & & \\
\hline preheat & 90 & 90 & 1300 & 120 & 6 & off & 4 & $0.5 / \mathrm{off}$ & 0/off & 0/on & -TA/on & \\
\hline 1 & 90 & 90 & 900 & 120 & 6 & Off & 4 & $0.5 /$ on & 0/off & 0/on & -TA/on & \\
\hline 2 & 90 & 90 & 900 & 120 & 6 & 30 & 4 & $0.5 / \mathrm{on}$ & 0/off & $0 /$ on & -TA/off & \\
\hline 3 & 90 & 50 & 800 & 120 & 6 & 30 & 4 & off & & $0 /$ on & off & \\
\hline 5 & 90 & 50 & 800 & 120 & 6 & 30 & 4 & off & & & off & \\
\hline 7 & 90 & 50 & 800 & 120 & 6 & 30 & 4 & off & & & off & \\
\hline 9 & 90 & 50 & 800 & 120 & 6 & 30 & 4 & off & & & off & \\
\hline 11 & 90 & 50 & 800 & 120 & 6 & 30 & 4 & off & & & off & \\
\hline 13 & 90 & 50 & 800 & 120 & 6 & 30 & 4 & off & & & off & \\
\hline 15 & 90 & 60 & 800 & 120 & 6 & 30 & 4 & off & & & off & \\
\hline 17 & 90 & 60 & 800 & 120 & 6 & 30 & 4 & off & & & off & \\
\hline 19 & 90 & 60 & 800 & 120 & 6 & 25 & 6 & off & & & off & \\
\hline 21 & 90 & 60 & 800 & 120 & 6 & 25 & 6 & off & & & off & \\
\hline 23 & 90 & 60 & 800 & 120 & 6 & 25 & 6 & off & & & off & \\
\hline 25 & 90 & 60 & 800 & 120 & 6 & 25 & 6 & off & & & off & \\
\hline 27 & 90 & 60 & 800 & 120 & 6 & 25 & 6 & off & & & off & \\
\hline 29 & 90 & 80 & 800 & 120 & 6 & 25 & 6 & off & & 2 & off & \\
\hline 31 & 90 & 80 & 800 & 120 & 6 & 20 & 8 & off & & 2 & off & \\
\hline 33 & 90 & 80 & 800 & 120 & 6 & 20 & 8 & off & & 2 & off & \\
\hline 35 & 90 & 80 & 900 & 120 & 6 & 20 & 8 & off & & ? & off & \\
\hline 37 & 90 & 80 & 900 & 120 & 6 & 20 & 8 & off & & 2.25 & off & \\
\hline $39-40$ & 90 & 140 & 1400 & Start & 6 & 0 & 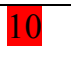 & off & off & 2.4 & off & \\
\hline
\end{tabular}

10.5 Dial copper carrier gas pressure to zero and close copper three way valve.

11. Increase torch parameters AS NEEDED to

$\begin{array}{lll}\text { Argon } & 90 \mathrm{psi} & 53 \text { orifice } \\ \text { Hydrogen } & 140 \mathrm{psi} & .009 \text { orifice }\end{array}$

Apply tungsten top coat for 70 minutes

12 Record temperature and time and number of passes

13 Cool until part reaches 500F

14 Turn cooling gases off 